\title{
Two-loop corrections to the large-order behavior of correlation functions in the one-dimensional $N$-vector model
}

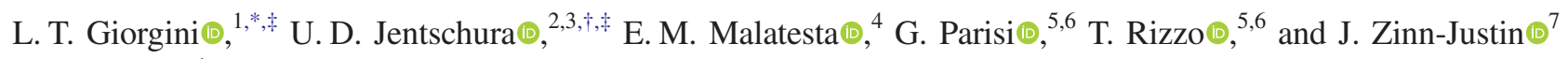 \\ ${ }^{1}$ Nordita, Royal Institute of Technology and Stockholm University, Stockholm 106 91, Sweden \\ ${ }^{2}$ Department of Physics, Missouri University of Science and Technology, Rolla, Missouri 65409, USA \\ ${ }^{3}$ MTA-DE Particle Physics Research Group, P.O. Box 51, H-4001 Debrecen, Hungary \\ ${ }^{4}$ Bocconi Institute for Data Science and Analytics, Bocconi University, Milano 20136, Italy \\ ${ }^{5}$ ISC-CNR, UOS Rome, Università “Sapienza," Piazzale A. Moro 2, I-00185 Rome, Italy \\ ${ }^{6}$ Dipartimento di Fisica, Università “Sapienza,” Piazzale A. Moro 2, I-00185 Rome, Italy \\ ${ }^{7}$ IRFU/CEA, Paris-Saclay, 91191 Gif-sur-Yvette Cedex, France
}

(Received 18 November 2019; accepted 28 April 2020; published 1 June 2020)

\begin{abstract}
For a long time, the predictive limits of perturbative quantum field theory have been limited by our inability to carry out loop calculations to an arbitrarily high order, which become increasingly complex as the order of perturbation theory is increased. This problem is exacerbated by the fact that perturbation series derived from loop diagram (Feynman diagram) calculations represent asymptotic (divergent) series which limits the predictive power of perturbative quantum field theory. Here, we discuss an ansatz that could overcome these limits, based on the observations that (i) for many phenomenologically relevant field theories, one can derive dispersion relations which relate the large-order growth (the asymptotic limit of "infinite loop order") with the imaginary part of arbitrary correlation functions, for negative coupling ("unstable vacuum"), and (ii) one can analyze the imaginary part for negative coupling in terms of classical field configurations (instantons). Unfortunately, the perturbation theory around instantons, which could lead to much more accurate predictions for the large-order behavior of Feynman diagrams, poses a number of technical as well as computational difficulties. Here, we study, to further the above-mentioned ansatz, correlation functions in a one-dimensional (1D) field theory with a quartic self-interaction and an $O(N)$ internal symmetry group, otherwise known as the 1D $N$-vector model. Our focus is on corrections to the large-order growth of perturbative coefficients, i.e., the limit of a large number of loops in the Feynman diagram expansion. We evaluate, in momentum space, the two-loop corrections for the two-point correlation function, and its derivative with respect to the momentum, as well as the two-point correlation function with a wigglet insertion. Also, we study the four-point function. These quantities, computed at zero momentum transfer, enter the renormalization-group functions (Callan-Symanzik equation) of the model. Our calculations pave the way for further development of related methods in field theory and for a better understanding of field-theoretical expansions at large order.
\end{abstract}

DOI: 10.1103/PhysRevD.101.125001

\section{INTRODUCTION}

\section{A. Orientation}

We here lay the groundwork for the detailed analysis of the large-order behavior of perturbation theory for correlation functions in field-theoretical models, pertaining to

*ludovico.giorgini@su.se

†ulj@mst.edu

${ }^{\ddagger}$ L. T. G. and U. D. J. contributed equally to this work.

Published by the American Physical Society under the terms of the Creative Commons Attribution 4.0 International license. Further distribution of this work must maintain attribution to the author(s) and the published article's title, journal citation, and DOI. Funded by SCOAP. phase transitions. Over the past decades, several steps have been made in the analysis of larger orders of perturbation theory, for both quantum mechanical problems as well as field theory. Indeed, it was Dyson who argued that, because of vacuum instabilities induced for a fictitiously negative value of the fine-structure constant, the quantum electrodynamic (QED) perturbation series could at best constitute an asymptotic series [1]. Later, this conjecture was substantiated, and the (factorial) divergence of perturbation theory, for both quantum mechanical oscillators [2-4] as well as field theory [5-8], was quantified both in terms of the power-law coefficients as well as in terms of the additive constants in the factorial growth of perturbation theory at large orders. Information regarding the leading terms in the perturbative expansion of perturbation theory 
has been instrumental in the determination of critical exponents for the $N$-vector model, which is a $\phi^{4}$ theory with an internal $O(N)$ symmetry group [9-12].

For anharmonic oscillators, one has been able to write down generalized Bohr-Sommerfeld quantization conditions that characterize the eigenvalues, including instanton contributions, to all orders [13-18]. From these conditions, one was able to infer the leading factorial divergence of perturbation theory, as well as subleading corrections, for large perturbation theory order. Calculations were, however, restricted to the partition function (i.e., to the groundstate energy of the quantum system).

However, a decisive step that has not been fully clarified in the literature so far is the extension of the large-order analysis beyond leading order to quantities of interest other than the partition function. Correlation functions are of interest in the calculation of critical exponents. First steps in this direction have been taken recently [19], with an emphasis on a scalar $\phi^{4}$ theory in two and three dimensions. Here, we report on essential progress in the latter endeavor, for a theory with an internal symmetry group $O(N)$, in one dimension. First, we use a formulation of the functional determinant [18], which allows us to separate the path integral around the nontrivial (instanton) saddle point into integrals over the collective coordinates (the start point of the instanton and the variables characterizing the internal space of the theory), as well as integrals over the transverse fluctuations around the nontrivial saddle point, in the internal symmetry group. The functional determinant does not factorize into longitudinal and transverse fluctuations (the latter being relevant to the internal space). Second, the application of the Wick theorem allows us to express the two-loop corrections around the classical extremum of the action, in terms of the longitudinal, and transverse, propagators of the (perpendicular) fluctuations, where "perpendicular" here refers to the exclusion of the zero mode, which is an eigenstate of the fluctuation operator with zero eigenvalue, corresponding to an invariance under a collective coordinate. Third, the final integrations are carried out and lead to expressions involving Riemann zeta functions of even and odd integer arguments. Eventually, we are able to carry out all integrations analytically. In the course of the calculations, we find the PSLQ algorithm useful in the very final analytic steps [20-23].

Here, we restrict the discussion to the one-dimensional case. We put special emphasis on the partition function, on the two-point correlation function, on its derivative with respect to the momentum, on the two-point correlation function with a wigglet insertion, and on the four-point correlation function. All the correlation functions are computed at zero momentum transfer, as is required for an input into the Callan-Symanzik equation. While, in one dimension, the field fluctuations are not strong enough to induce a phase transition, we clarify the connection of our calculations to the quantities entering the renormalizationgroup (RG) equations in Appendix A.

This paper is organized as follows. We derive the functional determinant for the transformation into collective coordinates and field fluctuations, for the quartic $O(N)$ theory, in Sec. II. The formalism is applied to the calculation of the imaginary part of the ground-state resonance energy (i.e., to the partition function in the large- $\beta$ limit). We use a normalization that makes the field equation for the instanton (classical) field configuration assume a particularly simple form [see Eq. (2.1) below]. The path integral Jacobian is derived with a particular emphasis on the nonfactorization of the longitudinal and transverse fluctuations. In Sec. III, we continue with the calculation of the two-point and four-point functions as well as the derivative of the two-point function at zero momentum transfer and the wigglet insertion. All of these functions enter the Callan-Symanzik [24,25] RG equations. Three appendixes complement our investigations. In Appendix A, we supply an integral table that is useful for the calculation of the propagator integrals. Appendix B is devoted to the connection of the correlation functions at zero momentum, investigated here, and the Callan-Symanzik equation.

\section{B. Large-order behavior and analyticity}

A central point of our investigations is the connection between the low-order behavior of the imaginary part of a $n$-point correlation function $\mathrm{G}(g)$ and the large-order behavior of its real part. Let us consider a generic Green function $\mathbf{G}(g)$ that is analytic in all the complex plane except on the negative real axis. We can apply the Cauchy theorem as follows:

$$
\mathrm{G}(g)=\frac{1}{2 \pi \mathrm{i}} \oint_{D} \mathrm{~d} z \frac{\mathrm{G}(z)}{z-g},
$$

where $D$ is the path in the complex plane encircling the branch cut on the negative real axis and $g$ is the reference argument where the function $\mathbf{G}(g)$ is to be evaluated. The path $\mathcal{D}$ can be decomposed into four contributions (see Fig. 1),

$$
\mathcal{D}=\mathcal{D}_{R}+\mathcal{D}_{\epsilon}+\mathcal{D}_{+}+\mathcal{D}_{-}
$$

The contributions over the paths $\mathcal{D}_{R}$ and $\mathcal{D}_{\epsilon}$ vanish identically, and the only remaining contributions come from the paths $\mathcal{D}_{+}$and $\mathcal{D}_{-}$. We can then write

$$
\begin{aligned}
\mathrm{G}(g) & =\frac{1}{2 \pi \mathrm{i}} \int_{-\infty}^{0} \mathrm{~d} z \frac{\operatorname{disc} \mathrm{G}(z)}{z-g}, \\
\operatorname{disc} \mathrm{G}(z) & =\lim _{\epsilon \rightarrow 0}[\mathrm{G}(z+\mathrm{i} \epsilon)-\mathrm{G}(z-\mathrm{i} \epsilon)] \\
& =-2 \mathrm{i} \operatorname{ImG}(z-\mathrm{i} \epsilon),
\end{aligned}
$$




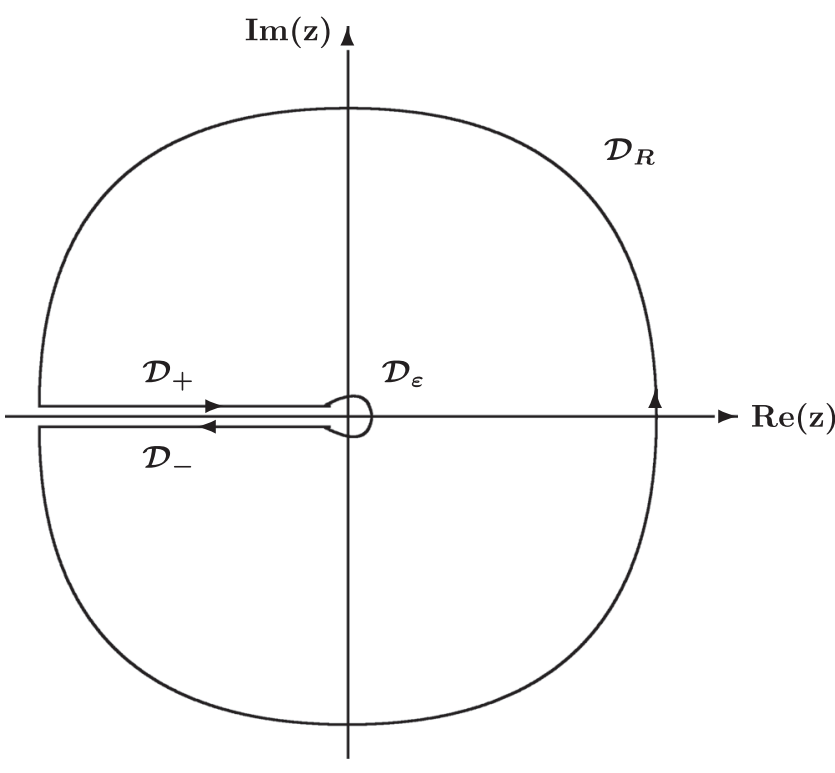

FIG. 1. The complex integration path $\mathcal{D}$ encircling the branch cut of the Green function.

where the discontinuity of $\mathrm{G}(z)$ on the cut is given by $\operatorname{disc} G(z)$. In the following, we will understand $G(z)$ for $z<0$ (on the cut) as the value of $\mathrm{G}(z)$ obtained when $z$ acquires an infinitesimal negative imaginary part. Expanding the relation (1.3) in $z$, we obtain

$$
\begin{aligned}
\mathrm{G}(g) & =\sum_{K=0}^{\infty} \mathrm{G}_{K} g^{K}=-\frac{1}{\pi} \int_{-\infty}^{0} \mathrm{~d} z \frac{\operatorname{ImG}(z-\mathrm{i} \epsilon)}{z-g} \\
& =-\frac{1}{\pi} \sum_{K=0}^{\infty} g^{K} \int_{-\infty}^{0} \mathrm{~d} z \frac{\operatorname{ImG}(z-\mathrm{i} \epsilon)}{z^{K+1}} .
\end{aligned}
$$

So, we find an integral representation for the perturbative coefficient of order $K$, of the $n$-point correlation function. The minus sign is consistent with Eq. (10) of Ref. [16] and with Eq. (2.31b) of Ref. [17]; note that, however, the resonance energy in the cited publications was identified with an infinitesimal positive imaginary part of the coupling.

We refer to the perturbative coefficient of order $K$ as $\mathrm{G}_{K}$ and write

$$
\begin{aligned}
\mathrm{G}_{K} & =-\frac{1}{\pi} \int_{-\infty}^{0} \mathrm{~d} g \frac{\operatorname{ImG}(g-\mathrm{i} \epsilon)}{g^{K+1}} \\
& =\frac{(-1)^{K}}{\pi} \int_{0}^{\infty} \mathrm{d} g \frac{\operatorname{ImG}(-g-\mathrm{i} \epsilon)}{g^{K+1}} .
\end{aligned}
$$

From this equation, we can understand the importance of knowing the value of the imaginary part of the correlation function for small and negative values of the coupling parameter $g$. In fact, the large order behavior of the series, i.e., $\mathrm{G}_{K}$ for $K$ large, is dominated by the values of $\operatorname{ImG}(g)$ at small and negative values of $g$.
In the following, we will find that the imaginary part of a generic n-point function $\mathrm{G}(g)$ involves, in leading order, a factor $(-g)^{(-N-1+D) / 2}$ from the leading-order Jacobian, given in Eq. (2.20). We anticipate that a factor $(-g)^{(-N-1) / 2}$ will be obtained from the $N-1$ collective coordinates inside the $O(N)$ symmetry group, which give rise to the $(N-1)$ th power of the classical field configuration in the Jacobian. In $D$ dimensions, one has $D$ additional collective coordinates describing translation invariance of the instanton in the $D$ spatial directions [8]. (For the current investigation, one has $D=1$.)

A further factor $(-g)^{-n / 2}$ stems from the $n$ classical field configurations in the $n$-point function. However, additional classical field configurations can be introduced into the leading-order expressions by mass derivatives, as is evident from the discussion of the wigglet insertion into the twopoint function (see Sec. III I). In general, our expressions for the imaginary part of a generic correlation function $\operatorname{ImG}(g)$ have the following structure:

$$
\begin{aligned}
\operatorname{Im} \mathrm{G}(g)= & c(N, D)(-g)^{-(n+N+D-1) / 2} \exp \left(\frac{A}{g}\right) \\
& \times\left[1+d(N, D) g+O\left(g^{2}\right)\right], \quad g<0,
\end{aligned}
$$

where $c(N, D)$ and $d(N, D)$ are constants and $n$ is the number of coordinates entering the Green function. We here concentrate on the Fourier transform. In one dimension, we find that $A=4 / 3$ in our conventions of the Euclidean action (2.1). Inserting Eq. (1.8) in Eq. (1.7), we get

$$
\begin{aligned}
\mathrm{G}_{K}= & c(N, D) \frac{(-1)^{K}}{\pi} \int_{0}^{\infty} \mathrm{d} g \frac{\mathrm{e}^{-A / g}}{g^{K+(n+N+D+1) / 2}}[1-g d(N, D)] \\
= & \frac{c(N, D)}{\pi} \Gamma(K+b)\left(\frac{1}{A}\right)^{(n+N+D-1) / 2}\left(-\frac{1}{A}\right)^{K} \\
& \times\left\{1-\frac{A d(N, D)}{K+b-1}\right\}, \quad b=\frac{n+N+D-1}{2}
\end{aligned}
$$

For large $K$, we can replace $K-1+b \rightarrow K$ in the denominator of the second term and identify the $1 / K$ correction. We also note the asymptotic expansion

$$
\frac{\Gamma(K+b)}{\Gamma(K+1)}=K^{b-1}\left[1+\frac{b(b-1)}{2 K}+\mathcal{O}\left(1 / K^{2}\right)\right]
$$

which can be used in order to bring the leading term in the expression (1.9) into the familiar form $C K^{b-1} B^{K} \Gamma(K+1)$, with suitable coefficients $C$ and $B$.

For our calculations as reported below, it is absolutely decisive to observe the connection of the perturbative correction about the instanton of relative order $g$, given by Eq. (1.8), and the subleading $1 / K$ correction to the leading factorial growth of the perturbative coefficients, 
given in Eq. (1.9). We shall evaluate the coefficients $d(N, D)$ by two-loop perturbation theory about the instanton configurations.

\section{QUARTIC THEORY WITH $O(N)$ SYMMETRY}

\section{A. Euclidean action}

We here follow Ref. [18] in the derivation of the $O(N)$ functional determinant, using a field normalization that allows us to express the field equations in a particularly simple analytic form. For the $O(N)$ one-dimensional field theory, we use the action in the form

$$
\begin{aligned}
\mathcal{S}[\underline{q}(t)] & =\int \mathrm{d} t\left[\frac{1}{2}\left(\frac{\partial \underline{q}(t)}{\partial t}\right)^{2}+\frac{1}{2} \underline{q}^{2}(t)+\frac{g}{4} \underline{q}^{4}(t)\right], \\
\underline{q}(t) & =\left\{q_{1}(t), \ldots, q_{N}(t)\right\}=q_{\alpha}(t) \underline{e}_{\alpha},
\end{aligned}
$$

where an $N$ vector in the internal space is denoted by underlining and, for completeness, we remark that $\underline{q}^{4}(t)$ is a shorthand notation for $\left[\underline{q}^{2}(t)\right]^{2}$. By group symmetry, for the classical field configuration, we can pick a specific direction $\underline{u}$ in the internal space, for the reference instanton configuration (note, however, that an averaging over the possible orientations of $\underline{u}$ is necessary at the end of the calculation, as discussed in the following). The classical field configuration is found as

$$
\underline{q}_{\mathrm{cl}}(t)=\underline{u} \sqrt{-\frac{1}{g}} \xi_{\mathrm{cl}}(t), \quad \xi_{\mathrm{cl}}(t)=\frac{\sqrt{2}}{\cosh (t)},
$$

which implies the existence of $N$ collective coordinates, namely, one time translation parametrized by $t_{0}$, and $N-1$ rotations in the internal space, leading to displacements orthogonal to the reference vector $\underline{u}$.

\section{B. Fluctuation operator}

By definition, the first functional derivative of the action with respect to $q_{\beta}(t)$,

$$
\left.\frac{\delta \mathcal{S}}{\delta q_{\beta}(t)}\right|_{\underline{q}=\underline{q}_{\mathrm{cl}}}=\left.\left(-\frac{\partial^{2}}{\partial t^{2}}+1+g q_{\gamma}(t) q_{\gamma}(t)\right) q_{\beta}(t)\right|_{\underline{q}=\underline{\mathrm{cl}}},
$$

vanishes at the classical path. This resulting equation is solved by Eq. (2.2), in view of the identity

$$
\left(-\frac{\partial^{2}}{\partial t^{2}}+1-\xi_{\mathrm{cl}}(t)^{2}\right) \xi_{\mathrm{cl}}(t)=0 .
$$

The second functional derivative at the classical path gives the fluctuation operator, for which we give a number of useful equivalent representations,

$$
\begin{aligned}
\mathbf{M}_{\alpha \beta}\left(t, t^{\prime}\right)= & \left.\frac{\delta \mathcal{S}}{\delta q_{\beta}(t) \delta q_{\alpha}\left(t^{\prime}\right)}\right|_{\underline{q}_{\underline{q}}} \\
= & \delta\left(t-t^{\prime}\right)\left[u_{\alpha} u_{\beta}\left(-\frac{\partial^{2}}{\partial t^{2}}+1-3 \xi_{\mathrm{cl}}^{2}(t)\right)\right. \\
& \left.+\delta_{\mathrm{T}, \alpha \beta}\left(-\frac{\partial^{2}}{\partial t^{2}}+1-\xi_{\mathrm{cl}}^{2}(t)\right)\right] \\
= & \delta\left(t-t^{\prime}\right)\left[u_{\alpha} u_{\beta} \mathbf{M}_{\mathrm{L}}(t)+\delta_{\mathrm{T}, \alpha \beta} \mathbf{M}_{\mathrm{T}}(t)\right] \\
= & \delta\left(t-t^{\prime}\right) \mathbf{M}_{\alpha \beta}(t) .
\end{aligned}
$$

Here, the transverse $\delta$ function is given as

$$
\delta_{\mathrm{T}, \alpha \beta}=\delta_{\alpha \beta}-u_{\alpha} u_{\beta},
$$

and we have defined the longitudinal (L) and transverse (T) fluctuation operators as

$$
\begin{aligned}
& \mathbf{M}_{\mathrm{L}}(t)=-\frac{\partial^{2}}{\partial t^{2}}+1-\frac{6}{\cosh ^{2}(t)} \\
& \mathbf{M}_{\mathrm{T}}(t)=-\frac{\partial^{2}}{\partial t^{2}}+1-\frac{2}{\cosh ^{2}(t)}
\end{aligned}
$$

The fluctuation operator $\mathbf{M}_{\mathrm{L}}=\mathbf{M}$ parametrizes the longitudinal fluctuations (in the initially chosen direction $\underline{u}$ of the instanton), whereas $\mathbf{M}_{\mathrm{T}}$ describes the transverse fluctuations (transverse to the initially chosen direction of the instanton). An illustrative remark is in order. We define the domain of the operators $\mathbf{M}_{\mathrm{L}}$ and $\mathbf{M}_{\mathrm{T}}$ so that respective zero modes are excluded. Thus, in our notation, the operators $\mathbf{M}_{\mathrm{L}}$ and $\mathbf{M}_{\mathrm{T}}$ are invertible. To denote the exclusion of the zero mode, the symbols $\mathbf{M}_{\mathrm{L}}^{\perp}$ and $\mathbf{M}_{\mathrm{T}}^{\perp}$ have been used in Ref. [18]. Because the longitudinal fluctuation operator fulfills $\mathbf{M}_{\mathrm{L}}=\mathbf{M}$, where $\mathbf{M}$ is the fluctuation operator for the scalar theory, we have $\boldsymbol{\Delta}_{\mathrm{L}}=\boldsymbol{\Delta}$. The inverse of $\mathbf{M}_{\alpha \beta}$ is $\boldsymbol{\Delta}_{\alpha \beta}$, with

$$
\boldsymbol{\Delta}_{\alpha \beta}=u_{\alpha} u_{\beta} \boldsymbol{\Delta}_{\mathrm{L}}+\delta_{\mathrm{T}, \alpha \beta} \boldsymbol{\Delta}_{\mathrm{T}} .
$$

The longitudinal and the transverse propagators $\boldsymbol{\Delta}_{\mathrm{L}}$ and $\boldsymbol{\Delta}_{\mathrm{T}}$ can be calculated analytically [18],

$$
\begin{aligned}
\Delta_{\mathrm{L}}\left(t_{1}, t_{2}\right)= & \frac{1}{4} \Theta\left(t_{1}-t_{2}\right) \frac{\sinh t_{1} \sinh t_{2}}{\cosh ^{2} t_{1} \cosh ^{2} t_{2}} f\left(t_{1}, t_{2}\right) \\
& +\left(t_{1} \leftrightarrow t_{2}\right), \\
f\left(t_{1}, t_{2}\right)= & 3 t_{2}-3 t_{1}-1+\mathrm{e}^{t_{2}} \frac{3 \sinh t_{2}-2 \cosh t_{2}}{\tanh t_{2}} \\
& +\mathrm{e}^{-t_{1}} \frac{3 \sinh t_{1}+2 \cosh t_{1}}{\tanh t_{1}}, \\
\Delta_{\mathrm{T}}\left(t_{1}, t_{2}\right)= & \frac{1}{4} \Theta\left(t_{1}-t_{2}\right) g\left(t_{1}, t_{2}\right)+\left(t_{1} \leftrightarrow t_{2}\right),
\end{aligned}
$$


$g\left(t_{1}, t_{2}\right)=\left(\frac{t_{2}-t_{1}-1}{\cosh t_{1} \cosh t_{2}}+\frac{\mathrm{e}^{-t_{1}}}{\cosh t_{2}}+\frac{\mathrm{e}^{t_{2}}}{\cosh t_{1}}\right)$.

A remark is in order. Namely, a comparison of Eqs. (2.3) to (2.7) reveals that the scalar instanton configuration $\xi_{\mathrm{cl}}(t)$ constitutes a zero mode of the transverse part of the fluctuation operator. The instanton path $\xi_{\mathrm{cl}}(t)$ has no zero. Therefore, when interpreted as a quantum mechanical wave function (eigenfunction of the fluctuation operator), it is clear that the instanton path represents the ground state of the transverse fluctuation operator. Thus, the ground state of the transverse fluctuation operator has zero eigenvalue, which implies that all other eigenvalues are manifestly greater than zero. The spectral determinant of the transverse fluctuation operator therefore is positive.

\section{Path integral Jacobian}

Even though the problem of the calculation of the functional determinant has been outlined in Ref. [18], we here revisit the derivation, with an emphasis on those aspects of the path integral Jacobian that are important for the calculation of correlation functions. The appropriate decomposition of the path reads as follows:

$$
\underline{q}(t)=\underline{u}\left[q_{\mathrm{cl}}\left(t-t_{0}\right)+\chi_{\mathrm{L}}\left(t-t_{0}\right)\right]+\underline{\chi}_{\mathrm{T}}\left(t-t_{0}\right),
$$

where $\underline{u}$ is a time-independent unit vector, $\underline{u}^{2}=1$, chosen to point into a specific direction of the $(N-1)$-dimensional unit sphere $S_{N-1}$ embedded in $N$-dimensional space. Furthermore, the longitudinal and transverse variations $\chi_{\mathrm{L}}(t)$ and $\chi_{\mathrm{T}}(t)$ are assumed to be orthogonal to their respective zero modes, i.e., $\underline{u} \cdot \underline{\chi}_{\mathrm{T}}\left(t-t_{0}\right)=0$. The variable $t_{0}$ takes the role of a collective coordinate. Throughout this paper, we denote vectors in the internal symmetry space by underlining.

To carry out the calculation (see Sec. 5 of Ref. [18]), one has to observe that the path decomposition (2.13), under the shift $t \rightarrow t-t_{0}$, breaks both time translation and $O(N)$ invariance, by singling out a specific direction $\underline{u}$ in the internal space as well as a start time $t_{0}$ for the instanton. The collective coordinates are the $N-1$ coordinates $\tau_{i}$ which parametrize the sphere $S_{N-1}$, as well as the time parameter $t_{0}$. One finds

$$
\begin{aligned}
\int & {[\mathrm{d} \underline{q}(t)] F[\underline{q}(t)] } \\
= & \left(\frac{1}{\sqrt{2 \pi}}\right)^{N} \int \mathrm{d} t_{0} \prod_{i=1}^{N-1} \int \mathrm{d} \tau_{i} \\
& \times \int\left[\mathrm{d} \chi_{\mathrm{L}}(t)\right] \int\left[\mathrm{d} \underline{\chi}_{\mathrm{T}}(t)\right] \mathcal{J}[\underline{q}(t)] \\
& \times F\left[\underline{u}\left[q_{\mathrm{cl}}\left(t-t_{0}\right)+\chi_{\mathrm{L}}\left(t-t_{0}\right)\right]+\underline{\chi}_{\mathrm{T}}\left(t-t_{0}\right)\right],
\end{aligned}
$$

where the Jacobian $\mathcal{J}(\underline{q})=\mathcal{J}[\underline{q}(t)]$ has the representation

$$
\begin{aligned}
& \mathcal{J}(\underline{q})=\frac{J(\underline{q})}{\sqrt{J\left(\underline{q}_{\mathrm{cl}}\right)}}, \\
& J(\underline{q})=\operatorname{det}\left(\int \mathrm{d} t \frac{\partial \underline{q}}{\partial c_{i}} \cdot \frac{\partial \underline{q}_{\mathrm{cl}}}{\partial c_{j}}\right), \quad c_{i}=\left(t_{0}, \tau_{i}\right) .
\end{aligned}
$$

The collective coordinates for time translations $t_{0}$ and the collective coordinates for rotations that parametrize $S_{N-1}$, which are denoted as $\tau_{i}(i=1, \ldots, N-1)$, are summarized in the vector $c_{i}$. It is crucial to carefully analyze the dependence on the collective coordinate $t_{0}$, for the path as well as the Jacobian, in the calculation of correlation functions. Furthermore, the identification of the path in terms of the argument $t-t_{0}$ (rather than $t+t_{0}$, as in Sec. 5 of Ref. [18]) serves to illustrate the role of $t_{0}$ as the "reference start point" of the classical path.

The rationale behind the transformation (2.14) is as follows. We start from the path integral over closed paths $\oint[\mathrm{d} q(t)]$. There are $N-1$ collective coordinates in the internal space of the $O(N)$ theory, and one collective coordinate describing the time translation of the longitudinal instanton. This means that there are $N$ collective coordinates in total; the exclusion of these from the remaining path integral leads to a factor $(2 \pi)^{-N / 2}$. In the remaining integral over the fluctuations $\oint\left[\mathrm{d} \chi_{\mathrm{L}}(t)\right]$, the longitudinal zero mode corresponding to the instanton path is excluded, leading to convergent expressions for the Gaussian path integral expectation values. The same applies to the integration over the transverse fluctuations $\oint\left[\mathrm{d} \underline{\chi}_{\mathrm{T}}(t)\right]$, where we exclude the transverse zero mode, to be discussed below, in all directions perpendicular to the fixed vector $\underline{u}$ in the internal space.

The path $q(t)$ is the sum of the classical path $\underline{u} q_{\mathrm{cl}}\left(t-t_{0}\right)$ and two sums over longitudinal fluctuations (L), and transverse fluctuations (T). The transverse fluctuations may point in any of the $N-1$ available directions. The $N-1$ vectors $\underline{e}_{2}, \ldots, \underline{e}_{N}$ parametrize the transverse fluctuations, orthogonal to $\underline{u}$ (where $\underline{u}$ can point into any direction in the internal space). We also set

$$
\underline{q}(t)=\underline{q}_{\mathrm{L}}\left(t-t_{0}\right)+\underline{q}_{\mathrm{T}}\left(t-t_{0}\right),
$$

with self-explanatory definitions for the longitudinal component $\underline{q}_{\mathrm{L}}\left(t-t_{0}\right)=\underline{u} q_{\mathrm{L}}\left(t-t_{0}\right)$ and the transverse component $\underline{q}_{\mathrm{T}}\left(t-t_{0}\right)$. The function $\dot{q}_{\mathrm{cl}}(t)$ is the zero mode of the longitudinal fluctuation operator, whereas $q_{\mathrm{cl}}(t)$ is the zero mode of the transverse fluctuation operator. The conditions that the zero modes should be omitted therefore read

$$
\int \mathrm{d} t \dot{q}_{\mathrm{cl}}(t)\left(q_{\mathrm{L}}(t)-q_{\mathrm{cl}}(t)\right)=0, \quad \int \mathrm{d} t q_{\mathrm{cl}}(t) \underline{q}_{\mathrm{T}}(t)=\underline{0},
$$


where the first condition comes from translations and the second from $O(N)$ rotations. The next step is to calculate the matrix elements relevant for the expression (2.15),

$$
\begin{aligned}
& J(\vec{q})=\operatorname{det}\left(\begin{array}{cc}
\mathcal{A} & \mathcal{B}^{\mathrm{T}} \\
\mathcal{C} & \mathcal{D}
\end{array}\right) \\
& =\left(\mathcal{A}-\mathcal{B}^{\mathrm{T}} \mathcal{D}^{-1} \mathcal{C}\right) \operatorname{det}(\mathcal{D}), \\
& \mathcal{A}=\int \mathrm{d} t \frac{\partial \underline{q}\left(t-t_{0}\right)}{\partial t_{0}} \cdot \frac{\partial \underline{q}_{\mathrm{cl}}\left(t-t_{0}\right)}{\partial t_{0}} \\
& =\int \mathrm{d} t \dot{q}_{\mathrm{L}}(t) \dot{q}_{\mathrm{cl}}(t), \\
& \mathcal{B}_{j}^{\mathrm{T}}=\int \mathrm{d} t \frac{\partial \underline{q}\left(t-t_{0}\right)}{\partial t_{0}} \cdot \frac{\partial \underline{q}_{\mathrm{cl}}\left(t-t_{0}\right)}{\partial \tau_{j}} \\
& =-\frac{\partial \underline{u}}{\partial \tau_{j}} \cdot \int \mathrm{d} t \underline{\dot{q}}_{\mathrm{T}}(t) q_{\mathrm{cl}}(t), \\
& \mathcal{C}_{i}=\int \mathrm{d} t \frac{\partial \underline{q}_{\mathrm{cl}}\left(t-t_{0}\right)}{\partial t_{0}} \cdot \frac{\partial \underline{q}\left(t-t_{0}\right)}{\partial \tau_{i}} \\
& =-\underline{u} \cdot \int \mathrm{d} t \dot{q}_{\mathrm{cl}}(t) \frac{\partial \underline{q}_{\mathrm{T}}(t)}{\partial \tau_{i}}, \\
& \mathcal{D}_{i j}=\int \mathrm{d} t \frac{\partial \underline{q}\left(t-t_{0}\right)}{\partial \tau_{i}} \cdot \frac{\partial \underline{q}_{\mathrm{cl}}\left(t-t_{0}\right)}{\partial \tau_{j}} \\
& =g_{i j} \int \mathrm{d} t q_{\mathrm{L}}(t) q_{\mathrm{cl}}(t) .
\end{aligned}
$$

Here, $\mathcal{B}^{\mathrm{T}}$ is a row vector, $\mathcal{C}$ is a column vector, $\mathcal{A}$ is a number, while $\mathcal{D}$ is an $(N-1) \times(N-1)$ matrix. We have introduced the metric

$$
g_{i j}=\frac{\partial \underline{u}}{\partial \tau_{i}} \cdot \frac{\partial \underline{u}}{\partial \tau_{j}}
$$

on the sphere $S_{N-1}$. We can write in the leading order,

$$
\begin{aligned}
\mathcal{J}[\underline{q}(t)] & \approx \sqrt{J\left[\underline{q}_{\mathrm{cl}}(t)\right]}=\sqrt{\operatorname{det}\left(g_{i j}\right)}\left\|\underline{q}_{\mathrm{cl}}\right\|^{N-1}\left\|\underline{\dot{q}}_{\mathrm{cl}}\right\| \\
& =\sqrt{\operatorname{det}\left(g_{i j}\right)}\left(-\frac{3 A}{g}\right)^{(N-1) / 2}\left(-\frac{A}{g}\right)^{1 / 2},
\end{aligned}
$$

where $\|f\|$ is the norm $\int_{-\infty}^{\infty} \mathrm{d} t f(t)$. A very useful representation is obtained upon division by the square root of the determinant of the metric in the internal space, which in view of Eq. (2.18a) is contained in the term $\operatorname{det}(\mathcal{D})$. One finds

$$
\begin{aligned}
\frac{\mathcal{J}[\underline{q}(t)]}{\left(\operatorname{det} g_{i j}\right)^{1 / 2}}= & \left(\frac{J\left[\underline{q}_{\mathrm{cl}}(t)\right]}{\operatorname{det} g_{i j}}\right)^{1 / 2} \frac{J[\underline{q}(t)]}{J\left[\underline{q}_{\mathrm{cl}}(t)\right]} \\
= & \left(-\frac{3 A}{g}\right)^{(N-1) / 2}\left(-\frac{A}{g}\right)^{1 / 2} \frac{J[\underline{q}(t)]}{J\left[\underline{q}_{\mathrm{cl}}(t)\right]}, \\
\frac{J[\underline{q}(t)]}{J\left[\underline{q}_{\mathrm{cl}}(t)\right]}= & \frac{\left(\int \mathrm{d} t \underline{q}(t) \cdot \underline{q}_{\mathrm{cl}}(t)\right)^{N-2}}{\left\|q_{\mathrm{cl}}(t)\right\|^{2(N-2)}} \frac{K}{\left\|\dot{q}_{\mathrm{cl}}(t)\right\|^{2}\left\|q_{\mathrm{cl}}(t)\right\|^{2}}, \\
K= & \int \mathrm{d} t \mathrm{~d} t^{\prime}\left[\underline{\dot{q}}(t) \cdot \underline{\dot{q}}_{\mathrm{cl}}(t) \underline{q}\left(t^{\prime}\right) \cdot \underline{q}_{\mathrm{cl}}\left(t^{\prime}\right)\right. \\
& \left.-\dot{q}_{\mathrm{cl}}(t) \dot{\dot{q}}_{\mathrm{cl}}\left(t^{\prime}\right) \underline{q}_{\mathrm{T}}(t) \cdot \underline{q}_{\mathrm{T}}\left(t^{\prime}\right)\right] .
\end{aligned}
$$

With the help of Eq. (2.14), we are now in the position to write the following identity:

$$
\begin{gathered}
\begin{aligned}
\int[\underline{\mathrm{q}}(t)] F[\underline{q}(t)] & =\left(\frac{1}{\sqrt{2 \pi}}\right)^{N} \sigma_{N} \int \mathrm{d} t_{0} \int\left[\mathrm{d} \chi_{\mathrm{L}}(t)\right] \\
& \times \int\left[\mathrm{d} \underline{\chi}_{\mathrm{T}}(t)\right]\left\langle\left(\frac{\mathcal{J}[\underline{Q}(t)]}{\left(\operatorname{det} g_{i j}\right)^{1 / 2}}\right) F(\underline{Q}(t))\right\rangle_{S_{N-1}},
\end{aligned} \\
\underline{Q}(t)=u\left(q_{\mathrm{cl}}\left(t-t_{0}\right)+\chi_{\mathrm{L}}\left(t-t_{0}\right)\right)+\underline{\chi}_{\mathrm{T}}\left(t-t_{0}\right), \\
\sigma_{N}=\prod_{i=1}^{N-1} \int \mathrm{d} \tau_{i}\left(\operatorname{det} g_{i j}\right)^{1 / 2}=\frac{2 \pi^{N / 2}}{\Gamma(N / 2)}, \\
\langle f(\underline{u})\rangle_{S_{N-1}}=\frac{1}{\sigma_{N}} \prod_{i=1}^{N-1} \int \mathrm{d} \tau_{i}\left(\operatorname{det} g_{i j}\right)^{1 / 2} f(\underline{u}) .
\end{gathered}
$$

Here, $\sigma_{N}$ is the surface of $S_{N-1}$, and the expression $\langle f(\underline{u})\rangle_{S_{N-1}}$ indicates the averaging of the test function $f(\underline{u})$ over the $S_{N-1}$ sphere. As an example for the averaging process, we indicate the formula $\left\langle u_{\alpha} u_{\beta}\right\rangle_{S_{N-1}}=\delta_{\alpha \beta} / N$. One important observation is that the path $\mathcal{J}[q(t)]$ in the Jacobian can be taken with a start time $t_{0}=0$ of the path. This is because all integrals contributing to the Jacobian are independent of $t_{0}$. However, the decomposition (2.13) is still valid; the path $q(t)$ depends on the start time $t_{0}$, and this dependence has to be figured into the integrand.

\section{D. $O(N)$ quartic oscillator}

Let us briefly review the calculation of the perturbative expansion of the ground-state energy for the $O(N)$ case, from the path integral representation. We write the Euclidean action as

$$
\begin{aligned}
\mathcal{S}[\underline{q}(t)]= & \int_{-\beta / 2}^{\beta / 2} \mathrm{~d} t_{1} \int_{-\beta / 2}^{\beta / 2} \mathrm{~d} t_{2} \underline{q}\left(t_{1}\right) \mathbf{M}_{0}\left(t_{1}, t_{2}\right) \underline{q}\left(t_{2}\right) \\
& +\frac{1}{4} g \int_{-\beta / 2}^{\beta / 2} \mathrm{~d} t \underline{q}(t)^{4},
\end{aligned}
$$


where the free fluctuation operator $\mathbf{M}_{0}$ and its inverse $\boldsymbol{\Delta}_{0}$ are given by

$$
\mathbf{M}_{0}\left(t_{1}, t_{2}\right)=\delta\left(t_{1}-t_{2}\right) \mathbf{M}_{0}\left(t_{2}\right), \quad \mathbf{M}_{0}(t)=-\frac{\partial^{2}}{\partial t^{2}}+1
$$

$\boldsymbol{\Delta}_{0} \cdot \mathbf{M}_{0}=\mathbb{1}, \quad \boldsymbol{\Delta}_{0}\left(t_{1}, t_{2}\right)=\frac{1}{2} \exp \left(-\left|t_{1}-t_{2}\right|\right)$.

One writes

$$
E_{0}(g)=\lim _{\beta \rightarrow \infty}\left(-\frac{1}{\beta} \ln \left(\frac{\mathcal{Z}_{0}(\beta)}{\left.\mathcal{Z}_{0}(\beta)\right|_{0}}\right)\right)+\frac{N}{2},
$$

where $\mathcal{Z}_{0}(\beta)$ is the saddle-point expansion of the partition function $\mathcal{Z}(\beta)$, redefined for the $O(N)$ oscillator, about the Gaussian saddle point, and $\left.\mathcal{Z}_{0}(\beta)\right|_{0}$ is obtained from $\mathcal{Z}_{0}(\beta)$ by setting $g=0$. The partition function can be written as follows:

$$
\left.\mathcal{Z}_{0}(\beta)\right|_{0}=\oint[\mathrm{d} \underline{q}(t)] \exp \left[-\frac{1}{2} \int \mathrm{d} t \int \mathrm{d} t^{\prime} \underline{q}(t) \mathbf{M}_{0}\left(t, t^{\prime}\right) \underline{q}\left(t^{\prime}\right)\right],
$$

where

$$
\oint[\mathrm{d} q(t)] \equiv \int_{-\infty}^{\infty} \mathrm{d} q_{0} \int_{q(-\beta / 2)=q_{0}}^{q(\beta / 2)=q_{0}}
$$

is a path integral over all periodic paths. We define a normalization factor

$$
\begin{aligned}
\mathcal{N} & =\int[\mathrm{d} \underline{q}(t)] \exp \left[-\frac{1}{2} \int \mathrm{d} t \int \mathrm{d} t^{\prime} \underline{q}(t) \mathbf{M}_{0}\left(t, t^{\prime}\right) \underline{q}\left(t^{\prime}\right)\right] \\
& =\frac{1}{\left(\operatorname{det} \mathbf{M}_{0}\right)^{N / 2}} .
\end{aligned}
$$

A perturbative expansion up to the order $g^{2}$ leads to the result

$$
\begin{aligned}
\frac{\mathcal{Z}_{0}(g)}{\left.\mathcal{Z}_{0}(\beta)\right|_{0}}= & 1-\frac{g}{4 \mathcal{N}} \int_{-\beta / 2}^{\beta / 2} \mathrm{~d} t \int[\mathrm{d} \underline{q}(t)][\underline{q}(t)]^{4} \mathcal{E}[\underline{q}(t)] \\
& +\frac{g^{2}}{32 \mathcal{N}} \int_{-\beta / 2}^{\beta / 2} \mathrm{~d} t \int_{-\beta / 2}^{\beta / 2} \mathrm{~d} t^{\prime} \\
& \times \int[\mathrm{d} q(t)] \underline{q}(t)^{4} \underline{q}\left(t^{\prime}\right)^{4} \mathcal{E}[\underline{q}(t)],
\end{aligned}
$$

where

$$
\mathcal{E}[\underline{q}(t)]=\exp \left(-\frac{1}{2} \int \mathrm{d} t_{1} \int \mathrm{d} t_{2} \underline{q}\left(t_{1}\right) \mathbf{M}_{0}\left(t_{1}, t_{2}\right) \underline{q}\left(t_{2}\right)\right) .
$$

We define the path integral expectation value $\langle Y\rangle_{0}$ as

$$
\langle Y\rangle_{0}=\left(\operatorname{det} \mathbf{M}_{0}\right)^{N / 2} \int[\mathrm{d} \underline{q}(t)] Y \mathcal{E}[\underline{q}(t)] .
$$

Application of the Wick theorem leads to

$$
\left\langle\underline{q}^{4}(t)\right\rangle_{0}=N(N+2)\left[\boldsymbol{\Delta}_{0}(0)\right]^{2}=\frac{1}{4} N(N+2),
$$

while the generalization to $\left\langle\underline{q}^{4}(t) \underline{q}\left(t^{\prime}\right)^{4}\right\rangle_{0}$ is straightforward. Finally, one obtains

$\ln \left(\frac{\mathcal{Z}_{0}(\beta)}{\left.\mathcal{Z}_{0}(\beta)\right|_{0}}\right)=-\frac{\beta g}{16} N(N+2)+\frac{\beta g^{2}}{128} N(N+2)(2 N+5)$,

$E_{0}(g)=\frac{N}{2}+\frac{g}{16} N(N+2)-\frac{g^{2}}{128} N(N+2)(2 N+5)$,

where we ignore terms of order $g^{3}$ and higher and confirm the cancellation of $\beta$ in the expression for $E_{0}(g)$.

\section{E. Decay width and instanton}

We are now in the position to present the analogous derivation of the leading-order result for the imaginary part of the ground-state resonance. The action (2.1), expressed in terms of the classical action plus fluctuations about the instanton configuration, becomes

$$
\begin{aligned}
\mathcal{S}[\underline{\chi}(t)]= & -\frac{4}{3 g}+\frac{1}{2} \int \mathrm{d} t_{1} \int \mathrm{d} t_{2} \chi_{\alpha}\left(t_{1}\right) \mathbf{M}_{\alpha \beta}\left(t_{1}, t_{2}\right) \chi_{\beta}\left(t_{2}\right) \\
& -\sqrt{-g} \int \mathrm{d} t \underline{\xi}_{\mathrm{cl}}(t) \cdot \underline{\chi}(t) \underline{\chi}^{2}(t)+\frac{g}{4} \int \mathrm{d} t \underline{\chi}^{4}(t) .
\end{aligned}
$$

For the calculation of the leading-order term in the decay width, we need the second term on the right-hand side, which is the term involving the fluctuation operator. We use Eqs. (2.14), as well as Eqs. (2.22a), (2.22c), and (2.22d). Observe that, for the partition function, we can simply integrate out the collective coordinate $\int \mathrm{d} t_{0}=\beta$. The leading contribution to the imaginary part $\operatorname{Im} E_{0}(g)$ for the ground-state energy of the $O(N)$ quartic oscillator is obtained as

$$
\begin{aligned}
\operatorname{Im} E_{0}(g) \approx & \lim _{\beta \rightarrow \infty}\left(-\frac{1}{\beta} \frac{\operatorname{Im} \mathcal{Z}_{1}(\beta)}{\mathcal{Z}_{0}(\beta)}\right) \\
= & -\frac{1}{\Gamma(N / 2)} \frac{1}{2^{N / 2}}\left(-\frac{4}{g}\right)^{N / 2} \frac{1}{\sqrt{3}} \exp \left(\frac{4}{3 g}\right) \\
& \times\left[-\operatorname{det}\left(\frac{1}{\mathbf{M}_{0}} \mathbf{M}_{\mathrm{L}}\right)\right]^{-1 / 2}\left[\operatorname{det}\left(\frac{1}{\mathbf{M}_{0}} \mathbf{M}_{\mathrm{T}}\right)\right]^{-\frac{1}{2}(N-1)} \\
= & -\frac{1}{\Gamma(N / 2)}\left(-\frac{8}{g}\right)^{N / 2} \exp \left(\frac{4}{3 g}\right)
\end{aligned}
$$


We resolve the ambiguity in taking the square root so that the imaginary part of the energy comes out as negative. The derivation in Eq. (2.36) implicitly supposes that $g$ is negative. As we saw in Sec. IB, the particular sign of the imaginary part chosen in Eq. (2.36) corresponds to values of $g$ with an infinitesimal negative imaginary part. In the derivation, we have used the results [18]

$\operatorname{det}\left(\frac{1}{\mathbf{M}_{0}} \mathbf{M}_{\mathrm{L}}\right)=-\frac{1}{12}, \quad \operatorname{det}\left(\frac{1}{\mathbf{M}_{0}} \mathbf{M}_{\mathrm{T}}\right)=\frac{1}{4}$.

\section{F. Corrected $O(N)$ decay width}

The key to the calculation of the corrections to the partition function, and (later on) to the corrections to the correlation functions, lies in the inclusion of corrections from three sources: (i) perturbative corrections from the expansion of the action (2.35), which enters the exponential $\exp (-\mathcal{S}[\underline{\chi}(t)])$, (ii) perturbative corrections from the expansion of the Jacobian $J[\underline{q}(t)] / J\left[\underline{q}_{\mathrm{cl}}(t)\right]$, and (iii) perturbative corrections from the denominator $\mathcal{Z}_{0}(\beta)$, in the expression $(-1 / \beta) \operatorname{Im}\left[\mathcal{Z}_{1}(\beta) / \mathcal{Z}_{0}(\beta)\right]$, in the limit of large $\beta$. An expansion of the exponential $\exp (-\mathcal{S}[\underline{\chi}(t)])$, according to Eq. (2.35), leads to the correction factor $F_{1}$,

$$
\begin{aligned}
F_{1}= & \frac{\mathrm{e}^{-\mathcal{S}[\underline{\chi}(t)]}}{\left.\mathrm{e}^{-\mathcal{S}[\underline{\chi}(t)]}\right|_{0}}=1+\sqrt{-g} \int \mathrm{d} t \underline{\xi}_{\mathrm{cl}}(t) \cdot \underline{\chi}(t) \underline{\chi}^{2}(t) \\
& -\frac{g}{4} \int \mathrm{d} t \underline{\chi}^{4}(t)-\frac{g}{2}\left(\int \mathrm{d} t \underline{\xi}_{\mathrm{cl}}(t) \cdot \underline{\chi}(t) \underline{\chi}^{2}(t)\right) \\
& \times\left(\int \mathrm{d} t^{\prime} \underline{\xi}_{\mathrm{cl}}\left(t^{\prime}\right) \cdot \underline{\chi}\left(t^{\prime}\right) \underline{\chi}^{2}\left(t^{\prime}\right)\right),
\end{aligned}
$$

where $\left.\mathcal{S}[\underline{\chi}(t)]\right|_{0}=\int \mathrm{d} t_{1} \int \mathrm{d} t_{2} \chi_{\alpha}\left(t_{1}\right) \mathbf{M}_{\alpha \beta}\left(t_{1}, t_{2}\right) \chi_{\beta}\left(t_{2}\right)$, which has to be inserted inside the path integral. The second factor $F_{2}$ is from the $O(N)$ Jacobian,

$$
\begin{aligned}
F_{2}= & \frac{J[\underline{q}(t)]}{J\left[\underline{q}_{\mathrm{cl}}(t)\right]}=1+\frac{3}{4} \sqrt{-g} \int \mathrm{d} t \underline{\dot{\chi}}(t) \cdot \underline{\dot{\xi}}_{\mathrm{cl}}(t) \\
& +(N-1) \frac{\sqrt{-g}}{4} \int \mathrm{d} t \underline{\chi}(t) \cdot \underline{\xi}_{\mathrm{cl}}(t) \\
& -\frac{3}{16}(N-1) g \int \mathrm{d} t \int \mathrm{d} t^{\prime} \underline{\underline{\chi}}(t) \cdot \underline{\xi}_{\mathrm{cl}}(t) \underline{\chi}\left(t^{\prime}\right) \cdot \underline{\xi}_{\mathrm{cl}}\left(t^{\prime}\right) \\
& -\frac{g}{32}(N-1)(N-2) \int \mathrm{d} t \int \mathrm{d} t^{\prime} \underline{\chi}_{(}(t) \cdot \underline{\xi}_{\mathrm{cl}}(t) \underline{\chi}^{\prime}\left(t^{\prime}\right) \cdot \underline{\xi}_{\mathrm{cl}}\left(t^{\prime}\right) \\
& +\frac{3}{16} g \int \mathrm{d} t \int \mathrm{d} t^{\prime} \dot{\xi}_{\mathrm{cl}}(t) \underline{\chi}_{\mathrm{T}}(t) \cdot \underline{\chi}_{\mathrm{T}}\left(t^{\prime}\right) \dot{\xi}_{\mathrm{cl}}\left(t^{\prime}\right) .
\end{aligned}
$$

Furthermore, there is a factor from the perturbative expansion of the denominator, which originates from the Gaussian saddle point (see Sec. II D),

$$
\begin{aligned}
F_{3} & =\frac{\left.\mathcal{Z}_{0}(\beta)\right|_{0}}{\mathcal{Z}_{0}(\beta)}=1+\frac{g}{4} \int \mathrm{d} t N(N+2)\left[\boldsymbol{\Delta}_{0}(0)\right]^{2} \\
& =1+\frac{g}{4} \int \mathrm{d} t\left[3+2(N-1)+\left(N^{2}-1\right)\right]\left[\boldsymbol{\Delta}_{0}(t, t)\right]^{2} .
\end{aligned}
$$

The latter form is very handy when it comes to subtracting infinities. The final result can be written as

$$
\begin{aligned}
\operatorname{Im} E_{0}(g) \approx & -\frac{1}{\Gamma(N / 2)}\left(-\frac{8}{g}\right)^{N / 2} \exp \left(\frac{4}{3 g}\right) \\
& \times(1+\mathcal{A}+\mathcal{B}+\mathcal{C}),
\end{aligned}
$$

where the terms $\mathcal{A}, \mathcal{B}$, and $\mathcal{C}$ are of order $g$, given by

$\mathcal{A}=\sum_{i=1}^{3} \mathcal{A}_{i}, \quad \mathcal{B}=\sum_{j=1}^{2} \mathcal{B}_{j}, \quad \mathcal{C}=\sum_{k=1}^{3} \mathcal{C}_{k}$

as defined in the following. (These are, of course, different from the submatrices $\mathcal{A}, \mathcal{B}$, and $\mathcal{C}$ used in Sec. II C; we redefine the symbols $\mathcal{A}, \mathcal{B}$, and $\mathcal{C}$ accordingly.) We distinguish the terms into $\mathcal{A}, \mathcal{B}$, and $\mathcal{C}$ as follows. The $\mathcal{A}$ originate from the effective action, i.e., from $F_{1}$, while infinities are removed by $F_{3}$. They correspond to the first three diagrams in Fig. 2. The $\mathcal{B}$ terms contain the mixed contributions from the product $F_{1} \times F_{2}$, expanded to order $(\sqrt{-g})^{2}=-g$ (see the fourth and fifth diagrams in Fig. 2). Terms of order $g$ in $F_{2}$ give rise to $\mathcal{C}$ (Jacobian terms; see the sixth, seventh, and eighth diagrams in Fig. 2).

We start with the $\mathcal{A}$ term,

$$
\begin{aligned}
\mathcal{A}= & -\frac{g}{4} \int \mathrm{d} t\left(\left\langle\underline{\chi}^{4}(t)\right\rangle-\left\langle\underline{\chi}^{4}(t)\right\rangle_{0}\right)-\frac{g}{2} \int \mathrm{d} t \int \mathrm{d} t^{\prime} \\
& \times\left\langle\underline{\xi}_{\mathrm{cl}}(t) \cdot \underline{\chi}(t) \underline{\chi}^{2}(t) \underline{\xi}_{\mathrm{cl}}\left(t^{\prime}\right) \cdot \underline{\chi}^{\prime}\left(t^{\prime}\right) \underline{\chi}^{2}\left(t^{\prime}\right)\right\rangle .
\end{aligned}
$$

We define $\langle\cdot\rangle$ for the $O(N)$ theory as

$$
\begin{aligned}
\langle X\rangle= & \left(\operatorname{det} \mathbf{M}_{\mathrm{L}}\right)^{1 / 2}\left(\operatorname{det} \mathbf{M}_{\mathrm{T}}\right)^{N / 2} \oint\left[\mathrm{d} \chi_{\mathrm{L}}(t)\right] \oint\left[\mathrm{d}_{\underline{\mathrm{T}}_{\mathrm{T}}}(t)\right] X \\
& \times \exp \left(-\frac{1}{2} \int \mathrm{d} t_{1} \int \mathrm{d} t_{2} \chi_{\alpha}\left(t_{1}\right) \mathbf{M}_{\alpha \beta}\left(t_{1}, t_{2}\right) \chi_{\beta}\left(t_{2}\right)\right) .
\end{aligned}
$$

The term $\mathcal{A}_{1}$ is easy,

$$
\begin{aligned}
\mathcal{A}_{1}= & -\frac{g}{4} \int \mathrm{d} t\left(\left\langle\chi_{\mathrm{L}}^{4}(t)\right\rangle+2\left\langle\chi_{\mathrm{L}}^{2}(t) \underline{\chi}_{\mathrm{T}}^{2}(t)\right\rangle+\left\langle\underline{\chi}_{\mathrm{T}}^{4}(t)\right\rangle\right) \\
& +\frac{g}{4} \int \mathrm{d} t\left\langle\underline{\chi}^{4}(t)\right\rangle_{0} .
\end{aligned}
$$

Applying the Wick theorem, we obtain the result 
(a)

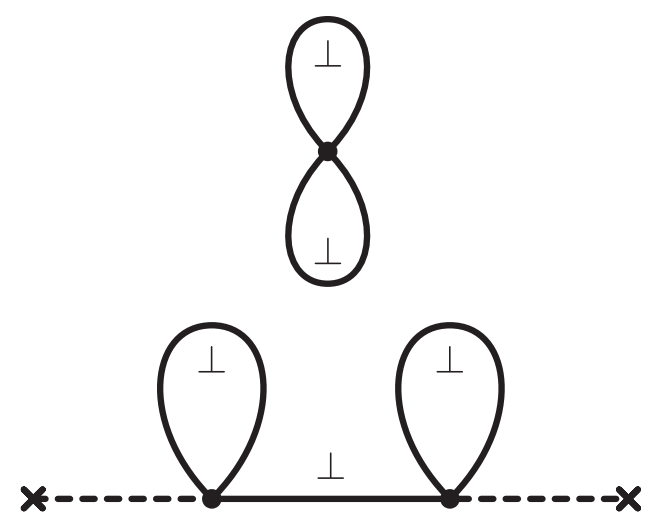

$$
\begin{aligned}
& \frac{5}{48} N^{2}+\frac{13}{40} N+\frac{59}{420} \\
& \frac{1}{48} N^{2}-\frac{11}{60} N-\frac{13}{70}
\end{aligned}
$$

(b)

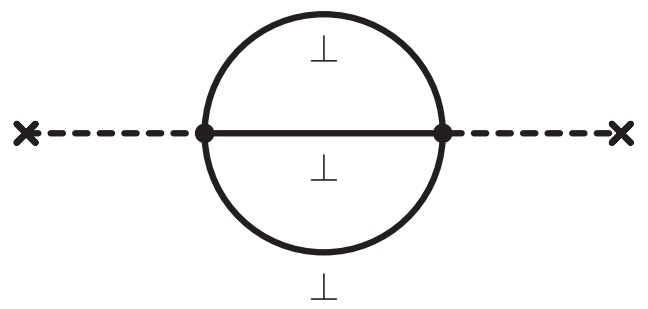

(c)

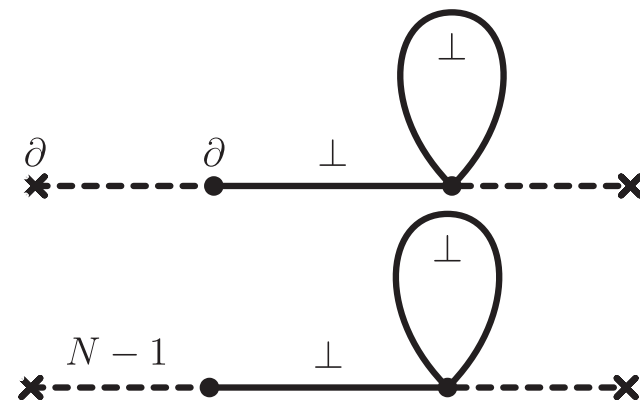

$$
-\frac{13}{480} N+\frac{223}{1680}
$$

(d)

(e)

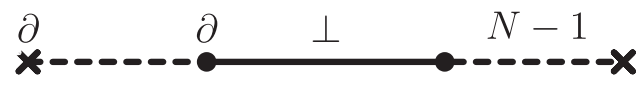

$$
\frac{5}{16} N+\frac{7}{20}
$$

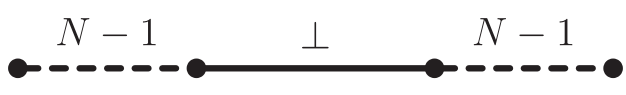

$$
\frac{1}{16} N^{2}-\frac{7}{48} N+\frac{1}{12}
$$

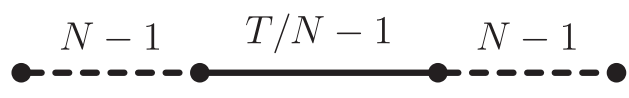

$$
\begin{aligned}
& \frac{3}{16} N-\frac{3}{16} \\
& \frac{1}{32} N^{2}-\frac{3}{32} N+\frac{1}{16} \\
& \frac{3}{16} N-\frac{3}{16}
\end{aligned}
$$

FIG. 2. Diagrammatic representation of several terms in a $\phi^{4}$ theory with an $O(N)$ internal symmetry, contributing to the partition function in the infinite- $\beta$ limit and thus, to the ground-state energy. The contribution of the diagrams is written beside each contribution. The total result of order $g$ is of the form given in Eq. (2.55).

$$
\begin{aligned}
\mathcal{A}_{1}= & -\frac{g}{4} \int \mathrm{d} t\left(3\left[\boldsymbol{\Delta}_{\mathrm{L}}^{2}(t, t)-\boldsymbol{\Delta}_{0}^{2}(t, t)\right]\right. \\
& +2(N-1)\left[\boldsymbol{\Delta}_{\mathrm{L}}(t, t) \boldsymbol{\Delta}_{\mathrm{T}}(t, t)-\boldsymbol{\Delta}_{0}^{2}(t, t)\right] \\
& \left.+\left(N^{2}-1\right)\left[\boldsymbol{\Delta}_{\mathrm{T}}^{2}(t, t)-\boldsymbol{\Delta}_{0}^{2}(t, t)\right]\right) \\
= & g\left(\frac{5}{48} N^{2}+\frac{13}{40} N+\frac{59}{420}\right) .
\end{aligned}
$$

For the second term in Eq. (2.43), one has

$$
\left\langle\underline{\xi}_{\mathrm{cl}}(t) \cdot \underline{\chi}(t) \underline{\chi}^{2}(t) \underline{\xi}_{\mathrm{cl}}\left(t^{\prime}\right) \cdot \underline{\chi}\left(t^{\prime}\right) \underline{\chi}^{2}\left(t^{\prime}\right)\right\rangle=T_{1}+T_{2},
$$

where

$$
\begin{aligned}
T_{1}= & \xi_{\mathrm{cl}}(t)\left[3 \Delta_{\mathrm{L}}(t, t)+(N-1) \Delta_{\mathrm{T}}(t, t)\right] \boldsymbol{\Delta}_{\mathrm{L}}\left(t, t^{\prime}\right) \\
& \times\left[3 \Delta_{\mathrm{L}}\left(t^{\prime}, t^{\prime}\right)+(N-1) \boldsymbol{\Delta}_{\mathrm{T}}\left(t^{\prime}, t^{\prime}\right)\right] \xi_{\mathrm{cl}}\left(t^{\prime}\right), \\
T_{2}= & 2 \xi_{\mathrm{cl}}(t) \Delta_{\mathrm{L}}\left(t, t^{\prime}\right)\left(3 \Delta_{\mathrm{L}}^{2}\left(t, t^{\prime}\right)\right. \\
& \left.+(N-1) \Delta_{\mathrm{T}}^{2}\left(t, t^{\prime}\right)\right) \xi_{\mathrm{cl}}\left(t^{\prime}\right) .
\end{aligned}
$$


The expression $T_{1}$ generates the term $\mathcal{A}_{2}$ [see the diagram in Fig. 2(b)], while the expression $T_{2}$ generates the term $\mathcal{A}_{3}$ [see the diagram in Fig. 2(c)],

$$
\begin{aligned}
& \mathcal{A}_{2}=g\left(\frac{1}{48} N^{2}-\frac{11}{60} N-\frac{13}{70}\right), \\
& \mathcal{A}_{3}=g\left(-\frac{13}{480} N+\frac{223}{1680}\right) .
\end{aligned}
$$

We now turn our attention to the $\mathcal{B}$ terms, which are generated by mixed contributions from $F_{1}$ and $F_{2}$. In fact, there are two terms in $F_{2}$ proportional to $\sqrt{-g}$, one of them being proportional to $(N-1)$. When multiplied by the term of order $\sqrt{-g}$ from $F_{1}$, these generate two mixed Feynman diagrams. The corresponding expression for the diagram in Fig. 2(d) reads

$$
\begin{aligned}
\mathcal{B}_{1} & =\frac{3 g}{4} \int \mathrm{d} t \int \mathrm{d} t^{\prime}\left\langle\underline{\dot{\chi}}(t) \cdot \underline{\dot{\xi}}_{\mathrm{cl}}(t) \underline{\xi}_{\mathrm{cl}}\left(t^{\prime}\right) \cdot \underline{\chi}\left(t^{\prime}\right) \underline{\chi}^{2}\left(t^{\prime}\right)\right\rangle \\
& =g\left(\frac{5}{16} N+\frac{7}{20}\right) .
\end{aligned}
$$

Furthermore, we have the expression for the diagram in Fig. 2(e),

$$
\begin{aligned}
\mathcal{B}_{2} & =(N-1) \frac{g}{4} \int \mathrm{d} t \int \mathrm{d} t^{\prime}\left\langle\underline{\chi}(t) \cdot \underline{\xi}_{\mathrm{cl}}(t) \underline{\xi}_{\mathrm{cl}}\left(t^{\prime}\right) \cdot \underline{\chi}(t) \underline{\chi}^{2}\left(t^{\prime}\right)\right\rangle \\
& =g\left(\frac{1}{16} N^{2}-\frac{7}{48} N+\frac{1}{12}\right) .
\end{aligned}
$$

There are three more terms generated by the terms of order $g$ in the $O(N)$ Jacobian. The first of these is given in Fig. 2(f) and reads

$$
\begin{aligned}
\mathcal{C}_{1} & =-\frac{3(N-1) g}{16} \int \mathrm{d} t \int \mathrm{d} t^{\prime}\left\langle\underline{\dot{\chi}}(t) \cdot \underline{\dot{\xi}}_{\mathrm{cl}}(t) \underline{\chi}\left(t^{\prime}\right) \cdot \underline{\xi}_{\mathrm{cl}}\left(t^{\prime}\right)\right\rangle \\
& =g\left(\frac{3}{16} N-\frac{3}{16}\right) .
\end{aligned}
$$

The diagram given in Fig. 2(g) gives rise to

$$
\begin{aligned}
\mathcal{C}_{2} & =-\frac{g}{32}(N-1)(N-2) \int \mathrm{d} t \int \mathrm{d} t^{\prime}\left\langle\underline{\chi}(t) \cdot \underline{\xi}_{\mathrm{cl}}(t) \underline{\chi}\left(t^{\prime}\right) \cdot \underline{\xi}_{\mathrm{cl}}\left(t^{\prime}\right)\right\rangle \\
& =g\left(\frac{1}{32} N^{2}-\frac{3}{32} N+\frac{1}{16}\right) .
\end{aligned}
$$

To complete the list, we analyze the diagram in Fig. 2(h),

$$
\begin{aligned}
\mathcal{C}_{3} & =\frac{3 g}{16} \int \mathrm{d} t \int \mathrm{d} t^{\prime}\left\langle\dot{\xi}_{\mathrm{cl}}(t) \underline{\chi}_{\mathrm{T}}(t) \cdot \underline{\chi}_{\mathrm{T}}\left(t^{\prime}\right) \dot{\xi}_{\mathrm{cl}}\left(t^{\prime}\right)\right\rangle \\
& =g\left(\frac{3}{16} N-\frac{3}{16}\right) .
\end{aligned}
$$

The result for the imaginary part of the $O(N)$ ground state resonance finally is obtained as

$$
\begin{aligned}
\operatorname{Im} E_{0}(g)= & -\frac{1}{\Gamma(N / 2)}\left(-\frac{8}{g}\right)^{N / 2} \exp \left(\frac{4}{3 g}\right) \\
& \times\left(1+\sum_{i=1}^{3} \mathcal{A}_{i}+\sum_{j=1}^{2} \mathcal{B}_{j}+\sum_{k=1}^{3} \mathcal{C}_{k}\right) \\
= & -\frac{1}{\Gamma(N / 2)}\left(-\frac{8}{g}\right)^{N / 2} \exp \left(\frac{4}{3 g}\right) \\
& \times\left[1+g\left(\frac{7}{32} N^{2}+\frac{9}{16} N+\frac{5}{24}\right)\right] .
\end{aligned}
$$

This result is relevant for $g<0$.

\section{CORRELATION FUNCTIONS}

\section{A. Leading-order contribution}

We turn to the evaluation of higher-order corrections to the imaginary part of correlation functions for negative $g$ and, thus, to the calculation of subleading corrections to the factorial growth of perturbative coefficients. The perturbative contribution exists for positive and negative coupling $g$; the cut across the negative $g$ axis is dominated by the instanton solution. The generating functional $\mathcal{Z}(J)$ of the correlation functions is given by

$$
\mathcal{Z}(\underline{J})=\frac{1}{\mathcal{N}} \int[\mathrm{d} \underline{q}(t)] \exp \left[-\mathcal{S}[\underline{q}(t)]+\int \mathrm{d} t \underline{J}(t) \cdot \underline{q}(t)\right],
$$

where both $\underline{J}(t)$ as well as $\underline{q}(t)$ are $N$ vectors. Note that $\mathcal{Z}(\underline{J})$ is not to be confused with the partition function $\mathcal{Z}(\beta)$. It is normalized so that, in leading order in $g$, and expanded about the Gaussian saddle point, one has $\mathcal{Z}(\underline{0}) \rightarrow 1$, i.e.,

$$
\begin{gathered}
\mathcal{N}=\int[\mathrm{d} \underline{q}(t)] \exp \left[-\mathcal{S}_{0}[\underline{q}(t)]\right], \\
\mathcal{S}_{0}[\underline{q}(t)]=\int \mathrm{d} t\left[\frac{1}{2}\left(\frac{\partial \underline{q}(t)}{\partial t}\right)^{2}+\frac{1}{2} \underline{q}^{2}(t)\right] .
\end{gathered}
$$

At leading order in the instanton contribution, the generating functional $\mathcal{Z}(J)$ is the sum of a perturbative expansion $\mathcal{Z}_{0}$ (about the Gaussian saddle point) and an imaginary, exponentially small contribution $\mathcal{Z}_{1}$ for $g \rightarrow 0$, which consists of the instanton contribution proportional to $\mathrm{e}^{A / g}$, for $g \rightarrow 0^{-}$,

$$
\begin{aligned}
\mathcal{Z}(\underline{J}) & =\mathcal{Z}_{0}(\underline{J})+\mathcal{Z}_{1}(\underline{J}), \\
\mathcal{W}(\underline{J}) & =\ln \mathcal{Z}(\underline{J})=\ln \mathcal{Z}_{0}(\underline{J})+\frac{\mathcal{Z}_{1}(\underline{J})}{\mathcal{Z}_{0}(\underline{J})} \\
& =\mathcal{W}_{0}(\underline{J})+\mathcal{W}_{1}(\underline{J}),
\end{aligned}
$$


where we note that $\mathcal{Z}_{1}(\underline{J})$ is exponentially suppressed for $g \rightarrow 0^{-}$. We note the implicit definitions $\mathcal{W}_{0}(\underline{J})=\ln \mathcal{Z}_{0}(\underline{J})$ and $\mathcal{W}_{1}(\underline{J})=\mathcal{Z}_{1}(\underline{J}) / \mathcal{Z}_{0}(\underline{J})$. The perturbative expansion defines $\mathcal{Z}_{0}(\underline{J})$ and holds irrespective of the sign of the coupling $g$. By contrast, the instanton contribution $\mathcal{Z}_{1}(\underline{J})$ is present only for negative $g$, and this is the implicit assumption on which all considerations reported in the current section are based. We investigate the connected $n$-point correlation functions $\mathcal{W}_{A,\left\{\alpha_{i}\right\}_{i=1}^{n}}^{(n)}$ and the complete $n$-point correlation functions $\mathcal{Z}_{A,\left\{\alpha_{i}\right\}_{i=1}^{n}}^{(n)}$ for $A=0$ (perturbative contributions) and $A=1$ (nonperturbative terms),

$$
\begin{aligned}
& \mathcal{W}_{A,\left\{\alpha_{i}\right\}_{i=1}^{n}}^{(n)}\left(t_{1}, \ldots, t_{n}\right)=\left.\left(\prod_{i=1}^{n} \frac{\delta}{\delta J_{\alpha_{i}}\left(t_{i}\right)}\right) \mathcal{W}_{A}(\underline{J})\right|_{\underline{J}=\underline{0}}, \\
& \mathcal{Z}_{A,\left\{\alpha_{i}\right\}_{i=1}^{n}}^{(n)}\left(t_{1}, \ldots, t_{n}\right)=\left.\left(\prod_{i=1}^{n} \frac{\delta}{\delta J_{\alpha_{i}}\left(t_{i}\right)}\right) \mathcal{Z}_{A}(\underline{J})\right|_{\underline{J}=\underline{0}} .
\end{aligned}
$$

To simplify the explicit expressions, we now assume that $\mathcal{S}(\underline{q})=\mathcal{S}(-\underline{q})$ and, thus, that correlation functions with $n$ odd vanish, which is certainly the case for our $\underline{q}^{4}$ model. Then, one finds, for example, for the zero-point function,

$\mathcal{W}_{1}(\underline{J}=\underline{0})=\frac{\mathcal{Z}_{1}(\underline{0})}{\mathcal{Z}_{0}(\underline{0})}, \quad \operatorname{Im} \mathcal{W}_{1}(\underline{J}=\underline{0})=\frac{\operatorname{Im} \mathcal{Z}_{1}(\underline{0})}{\mathcal{Z}_{0}(\underline{0})}$.

For the two-point function, one finds

$$
\begin{aligned}
\mathcal{W}_{1, \alpha_{1} \alpha_{2}}^{(2)}\left(t_{1}, t_{2}\right) & =\left.\frac{\delta^{2}}{\delta J_{\alpha_{1}}\left(t_{1}\right) \delta J_{\alpha_{2}}\left(t_{2}\right)} \mathcal{W}_{1}(\underline{J})\right|_{\underline{J}=\underline{0}} \\
& =\frac{\mathcal{Z}_{1, \alpha_{1} \alpha_{2}}^{(2)}\left(t_{1}, t_{2}\right)}{\mathcal{Z}_{0}(\underline{0})}-\frac{\mathcal{Z}_{0, \alpha_{1} \alpha_{2}}^{(2)}\left(t_{1}, t_{2}\right) \mathcal{Z}_{1}(\underline{0})}{\mathcal{Z}_{0}^{2}(\underline{0})},
\end{aligned}
$$

and the imaginary part of the two-point function is obtained as follows:

$$
\begin{aligned}
\operatorname{Im} \mathcal{W}_{1, \alpha_{1} \alpha_{2}}^{(2)}\left(t_{1}, t_{2}\right)= & \frac{\operatorname{Im} \mathcal{Z}_{1, \alpha_{1} \alpha_{2}}^{(2)}\left(t_{1}, t_{2}\right)}{\mathcal{Z}_{0}(\underline{0})} \\
& -\frac{\mathcal{Z}_{0, \alpha_{1} \alpha_{2}}^{(2)}\left(t_{1}, t_{2}\right) \operatorname{Im} \mathcal{Z}_{1}(\underline{0})}{\mathcal{Z}_{0}^{2}(\underline{0})} .
\end{aligned}
$$

Furthermore, we can express the imaginary part of the fourpoint function as a sum of four terms $\mathcal{K}_{i}(i=1, \ldots, 4)$,

$$
\operatorname{Im} \mathcal{W}_{1, \alpha_{1} \alpha_{2} \alpha_{3} \alpha_{4}}^{(4)}\left(t_{1}, t_{2}, t_{3}, t_{4}\right)=\sum_{i=1}^{4} \mathcal{K}_{i} .
$$

The first term involves the imaginary part of the four-point instanton contribution $\operatorname{Im} \mathcal{Z}_{1, \alpha_{1} \alpha_{2} \alpha_{3} \alpha_{4}}^{(4)}$,

$$
\mathcal{K}_{1}=\frac{\operatorname{Im} \mathcal{Z}_{1, \alpha_{1} \alpha_{2} \alpha_{3} \alpha_{4}}^{(4)}\left(t_{1}, t_{2}, t_{3}, t_{4}\right)}{\mathcal{Z}_{0}(\underline{0})} .
$$

The second term is a mixed term, involving two-point perturbative and two-point instanton correlation functions,

$$
\begin{aligned}
\mathcal{K}_{2}= & -\frac{\mathcal{Z}_{0, \alpha_{1} \alpha_{2}}^{(2)}\left(t_{1}, t_{2}\right) \operatorname{Im} \mathcal{Z}_{1, \alpha_{3} \alpha_{4}}^{(2)}\left(t_{3}, t_{4}\right)}{\mathcal{Z}_{0}^{2}(\underline{0})}-\frac{\mathcal{Z}_{0, \alpha_{1} \alpha_{3}}^{(2)}\left(t_{1}, t_{3}\right) \operatorname{Im} \mathcal{Z}_{1, \alpha_{2} \alpha_{4}}^{(2)}\left(t_{2}, t_{4}\right)}{\mathcal{Z}_{0}^{2}(\underline{0})}-\frac{\mathcal{Z}_{0, \alpha_{1} \alpha_{4}}^{(2)}\left(t_{1}, t_{4}\right) \operatorname{Im} \mathcal{Z}_{1, \alpha_{2} \alpha_{3}}^{(2)}\left(t_{2}, t_{3}\right)}{\mathcal{Z}_{0}^{2}(\underline{0})} \\
& -\frac{\mathcal{Z}_{0, \alpha_{3} \alpha_{4}}^{(2)}\left(t_{3}, t_{4}\right) \operatorname{Im} \mathcal{Z}_{1, \alpha_{1} \alpha_{2}}^{(2)}\left(t_{1}, t_{2}\right)}{\mathcal{Z}_{0}^{2}(\underline{0})}-\frac{\mathcal{Z}_{0, \alpha_{2} \alpha_{4}}^{(2)}\left(t_{2}, t_{4}\right) \operatorname{Im} \mathcal{Z}_{1, \alpha_{1} \alpha_{3}}^{(2)}\left(t_{1}, t_{4}\right)}{\mathcal{Z}_{0}^{2}(\underline{0})}-\frac{\mathcal{Z}_{0, \alpha_{2} \alpha_{3}}^{(2)}\left(t_{2}, t_{3}\right) \operatorname{Im} \mathcal{Z}_{1, \alpha_{1} \alpha_{4}}^{(2)}\left(t_{1}, t_{4}\right)}{\mathcal{Z}_{0}^{2}(\underline{0})} .
\end{aligned}
$$

The third term combines the four-point perturbative correlation function with the imaginary part of the zero-point function,

$$
\mathcal{K}_{3}=-\frac{\mathcal{Z}_{0, \alpha_{1} \alpha_{2} \alpha_{3} \alpha_{4}}^{(4)}\left(t_{1}, t_{2}, t_{3}, t_{4}\right) \operatorname{Im} \mathcal{Z}_{1}(\underline{0})}{\mathcal{Z}_{0}^{2}(\underline{0})} .
$$

Note that $\operatorname{Im} \mathcal{Z}_{1}(\underline{0})$ is equal to the imaginary part of the partition function, up to a factor $\beta$. Finally, the fourth term involves two perturbative two-point functions,

$$
\begin{aligned}
\mathcal{K}_{4}= & \frac{2 \mathcal{Z}_{0, \alpha_{1} \alpha_{2}}^{(2)}\left(t_{1}, t_{2}\right) \mathcal{Z}_{0, \alpha_{3} \alpha_{4}}^{(2)}\left(t_{3}, t_{4}\right)}{\mathcal{Z}_{0}^{3}(\underline{0})} \operatorname{Im} \mathcal{Z}_{1}(\underline{0}) \\
& +\frac{2 \mathcal{Z}_{0, \alpha_{1} \alpha_{3}}^{(2)}\left(t_{1}, t_{3}\right) \mathcal{Z}_{0, \alpha_{2} \alpha_{4}}^{(2)}\left(t_{2}, t_{4}\right)}{\mathcal{Z}_{0}^{3}(\underline{0})} \operatorname{Im} \mathcal{Z}_{1}(\underline{0}) \\
& +\frac{2 \mathcal{Z}_{0, \alpha_{1} \alpha_{4}}^{(2)}\left(t_{1}, t_{4}\right) \mathcal{Z}_{0, \alpha_{2} \alpha_{3}}^{(2)}\left(t_{2}, t_{3}\right)}{\mathcal{Z}_{0}^{3}(\underline{0})} \operatorname{Im} \mathcal{Z}_{1}(\underline{0}) .
\end{aligned}
$$

At leading order, $\operatorname{Im} \mathcal{Z}_{1}^{(n)}$ is proportional to $\left(q_{\mathrm{cl}}\right)^{n}$. The classical path $q_{\mathrm{cl}}$ is of order $1 / \sqrt{-g}$. So, the imaginary part $\operatorname{Im} \mathcal{Z}_{1}^{(n)}$ of the $n$-point function is of order $(-g)^{-n / 2} \operatorname{Im} \mathcal{Z}_{1}(\underline{0})$. 
This implies the inequality $\operatorname{Im} \mathcal{Z}_{1}^{(4)} \gg \operatorname{Im} \mathcal{Z}_{1}^{(2)} \gg \operatorname{Im} \mathcal{Z}_{1}$, and thus, at leading order, the disconnected parts are suppressed. Finally, a generic expression for the connected $n$-point Green function is given by

$$
\begin{aligned}
\mathrm{G}_{\left\{\alpha_{i}\right\}_{i=1}^{n}}^{(n)}\left(t_{1}, \ldots, t_{n}\right)= & \left.\left(\prod_{i=1}^{n} \frac{\delta}{\delta J_{\alpha_{i}}\left(t_{i}\right)}\right) \mathcal{W}(\underline{J})\right|_{\underline{J}=\underline{0}} \\
= & \mathcal{W}_{0,\left\{\alpha_{i}\right\}_{i=1}^{n}}^{(n)}\left(t_{1}, \ldots, t_{n}\right) \\
& +\mathcal{W}_{1,\left\{\alpha_{i}\right\}_{i=1}^{n}}^{(n)}\left(t_{1}, \ldots, t_{n}\right),
\end{aligned}
$$

where $\mathcal{W}$ is the sum of $\mathcal{W}_{0}$ and $\mathcal{W}_{1}$. The definition encompasses both the real and the imaginary parts of the $n$-point correlation function [see Eq. (3.3a)]. For the imaginary part, we define

$$
\begin{aligned}
\mathcal{G}_{\left\{\alpha_{i}\right\}_{i=1}^{n}}^{(n)}\left(t_{1}, \ldots, t_{n}\right) & =\operatorname{ImG}_{\left\{\alpha_{i}\right\}_{i=1}^{n}}^{(n)}\left(t_{1}, \ldots, t_{n}\right) \\
& =\operatorname{Im} \mathcal{W}_{1,\left\{\alpha_{i}\right\}_{i=1}^{n}}^{(n)}\left(t_{1}, \ldots, t_{n}\right) .
\end{aligned}
$$

\section{B. Two-point correlation function}

We investigate the (imaginary part of the) two-point Green function $\mathcal{G}_{\alpha \beta}\left(t_{1}, t_{2}\right)$, according to Eq. (3.14), as follows:

$$
\begin{aligned}
\mathcal{G}_{\alpha \beta}\left(t_{1}, t_{2}\right) & =\frac{\operatorname{Im} \mathcal{Z}_{1, \alpha \beta}^{(2)}\left(t_{1}, t_{2}\right)}{\mathcal{Z}_{0}(\underline{0})}-\frac{\mathcal{Z}_{0, \alpha \beta}^{(2)}\left(t_{1}, t_{2}\right) \operatorname{Im} \mathcal{Z}_{1}(\underline{0})}{\mathcal{Z}_{0}^{2}(\underline{0})} \\
& =\left[G_{\alpha \beta}\left(t_{1}, t_{2}\right)\right]_{1}+\left[G_{\alpha \beta}\left(t_{1}, t_{2}\right)\right]_{2} \\
& \approx\left[G_{\alpha \beta}\left(t_{1}, t_{2}\right)\right]_{1} .
\end{aligned}
$$

Note that in the last step of the previous expression, we have reported only the leading term in $g$; however, $\left[G_{\alpha \beta}\left(t_{1}, t_{2}\right)\right]_{2}$ will be important when we will consider the first subleading order.

It is useful to remark that all $\mathcal{Z}$ quantities are now understood in the sense of Eq. (3.1), i.e., without $\int \mathrm{d} q_{0}$. We need to evaluate an integral of the form $\oint[\mathrm{d} q(t)] F[\underline{q}(t)]$ with the help of Eq. (2.22), where in leading order in $g$, one has

$$
\begin{aligned}
F[\underline{q}(t)] & =q_{\mathrm{cl}, \alpha}\left(t_{1}\right) q_{\mathrm{cl}, \beta}\left(t_{2}\right) \\
& \approx-\frac{1}{g} u_{\alpha} u_{\beta} \xi_{\mathrm{cl}}\left(t_{1}-t_{0}\right) \xi_{\mathrm{cl}}\left(t_{2}-t_{0}\right), \\
\langle F[\underline{q}(t)]\rangle_{S_{N-1}} & \approx-\frac{1}{g}\left\langle u_{\alpha} u_{\beta}\right\rangle_{S_{N-1}} \xi_{\mathrm{cl}}\left(t_{1}-t_{0}\right) \xi_{\mathrm{cl}}\left(t_{2}-t_{0}\right) \\
& =-\frac{1}{g} \frac{\delta_{\alpha \beta}}{N} \xi_{\mathrm{cl}}\left(t_{1}-t_{0}\right) \xi_{\mathrm{cl}}\left(t_{2}-t_{0}\right) .
\end{aligned}
$$

We recall that, according to the remarks surrounding Eq. (2.22c), we need to supplement the collective coordinate $t_{0}$ in the actual path. Hence, we can approximate, in leading order,

$$
\begin{aligned}
\mathcal{G}_{\alpha \beta}\left(t_{1}, t_{2}\right) \approx & \left(\frac{1}{\sqrt{2 \pi}}\right)^{N} \frac{2 \pi^{N / 2}}{\Gamma(N / 2)}\left(\frac{1}{2}\right)\left(-\frac{4}{g}\right)^{(N-1) / 2}\left(-\frac{4}{3 g}\right)^{1 / 2} \exp \left(\frac{4}{3 g}\right)\left[-\operatorname{det}\left(\frac{1}{\mathbf{M}_{0}} \mathbf{M}_{\mathrm{L}}\right)\right]^{-1 / 2}\left[\operatorname{det}\left(\frac{1}{\mathbf{M}_{0}} \mathbf{M}_{\mathrm{T}}\right)\right]^{-N / 2} \\
& \times\left(-\frac{1}{g} \frac{\delta_{\alpha \beta}}{N} \int \mathrm{d} t_{0} \xi_{\mathrm{cl}}\left(t_{1}-t_{2}-t_{0}\right) \xi_{\mathrm{cl}}\left(-t_{0}\right)\right) \\
= & -\frac{1}{g} \frac{1}{\Gamma(N / 2)} \frac{\delta_{\alpha \beta}}{N}\left(-\frac{8}{g}\right)^{N / 2} \exp \left(\frac{4}{3 g}\right) \frac{4\left(t_{1}-t_{2}\right)}{\sinh \left(t_{1}-t_{2}\right)}
\end{aligned}
$$

This imaginary part $\mathcal{G}_{\alpha \beta}\left(t_{1}, t_{2}\right)$ is positive for negative $g$, in contrast to the negative imaginary part of the groundstate resonance energy. [We recall that, according to Eq. (3.15), the imaginary part of the Green function itself, not the entire Green function, is denoted as $\mathcal{G}_{\alpha \beta}\left(t_{1}, t_{2}\right)$.]

\section{Some observations}

Before we go in medias res, four observations should be made.

(i) We are interested in the corrections to the result (3.18) of relative order $g$. One of these corrections can be obtained almost automatically, by observing that the derivation of the leading term given in Eq. (3.18) does not entail path integrals except in leading order; the product of the two classical field configurations $\int \mathrm{d} t_{0} \xi_{\mathrm{cl}}\left(t_{1}-t_{2}-t_{0}\right) \xi_{\mathrm{cl}}\left(-t_{0}\right)$ simply drops out as a prefactor of the integral. Hence, the two-point correlation function receives the same relative correction as the partition function itself; i.e., it has to be multiplied by [see Eq. (2.55)]

$$
\begin{aligned}
\mathcal{F}_{\mathcal{Z}} & =\left(\frac{1}{\beta} \frac{\operatorname{Im} \mathcal{Z}(\beta)}{\mathcal{Z}_{0}(\beta)}\right)\left[\left.\left(\frac{1}{\beta} \frac{\operatorname{Im} \mathcal{Z}(\beta)}{\mathcal{Z}_{0}(\beta)}\right)\right|_{0}\right]^{-1} \\
& =1+g\left(\frac{7}{32} N^{2}+\frac{9}{16} N+\frac{5}{24}\right),
\end{aligned}
$$


where, with the subscript zero, we denote the leading contribution to the imaginary part of the groundstate energy. We note that in the two-point correlation function, the integration over the collective coordinate $\int \mathrm{d} t_{0}$ is carried out over the arguments of the classical field configuration; in the derivation of the partition function, by contrast, it leads to a factor $\int \mathrm{d} t=\beta$, which is later divided out in calculating the energy. We note that the leading term in the two-point correlation function can be written as follows,

$$
\begin{aligned}
\mathcal{G}_{\alpha \beta}\left(t_{1}, t_{2}\right) \approx & \left.\left(\frac{1}{\beta} \frac{\operatorname{Im} \mathcal{Z}_{1}(\beta)}{\mathcal{Z}_{0}(\beta)}\right)\right|_{0} \\
& \times\left(-\frac{1}{g} \frac{\delta_{\alpha \beta}}{N} \int \mathrm{d} t_{0} \xi_{\mathrm{cl}}\left(t_{1}-t_{2}-t_{0}\right) \xi_{\mathrm{cl}}\left(-t_{0}\right)\right) .
\end{aligned}
$$

Replacing the prefactor according to

$$
\left.\left(\frac{1}{\beta} \frac{\operatorname{Im} \mathcal{Z}(\beta)}{\mathcal{Z}_{0}(\beta)}\right)\right|_{0} \rightarrow \frac{1}{\beta} \frac{\operatorname{Im} \mathcal{Z}(\beta)}{\mathcal{Z}_{0}(\beta)}
$$

takes care of the correction, and that replacement exactly amounts to the multiplication of the leadingorder result by the correction factor $\mathcal{F}_{\mathcal{Z}}$.

(ii) The angular symmetry of the problem implies that

$$
\mathcal{G}_{\alpha \beta}\left(t_{1}, t_{2}\right)=\frac{\delta_{\alpha \beta}}{N} \mathcal{G}_{\gamma \gamma}\left(t_{1}, t_{2}\right) .
$$

Hence, we can restrict the discussion, in the following, to the function

$$
\mathcal{G}\left(t_{1}, t_{2}\right)=\mathcal{G}_{\gamma \gamma}\left(t_{1}, t_{2}\right)=\delta_{\alpha \beta} \mathcal{G}_{\alpha \beta}\left(t_{1}, t_{2}\right),
$$

an operation that also eliminates the necessity to do angular averaging.

(iii) We recall the action factor $F_{1}$ from Eq. (2.38), the Jacobian factor $F_{2}$ from Eq. (2.39), and the perturbative factor $F_{3}$ from Eq. (2.40). The correction due to the factor $F_{3}$, in relative order $g$, is already taken into account in the denominator of the perturbative partition function $\mathcal{Z}_{0}(\beta)$ in the replacement in Eq. (3.21). If we are thinking about the calculation of perturbative corrections about the instanton saddle point of the two-point correlation function, then we must consider that the leading term is proportional to

$$
\left\langle q_{\mathrm{cl}, \alpha}\left(t_{1}-t_{0}\right) q_{\mathrm{cl}, \beta}\left(t_{2}-t_{0}\right)\right\rangle_{S_{N-1}},
$$

according to Eq. (3.18). Corrections of relative order $g$ are obtained in two ways, first, by replacing, in Eq. (3.24), both classical paths by fluctuations, which results in a term of relative order $g$, because the fluctuations are of order $g^{0}$, while the classical field configurations are of order $1 / \sqrt{-g}$. In this case, the calculation proceeds simply by evaluating the path integral, without any further perturbative corrections from either $F_{1}$ or $F_{2}$, and is already of the required relative order $g$.

The second way to obtain a correction of relative order $g$ is to replace only one of the classical field configurations in Eq. (3.24) by a fluctuation, and to contract the remaining term with the term $\mathcal{F}_{\mathcal{J}}$ which contains the terms up to relative order $\sqrt{-g}$ from the product $F_{1} F_{2}$ and reads as follows:

$$
\begin{aligned}
\mathcal{F}_{\mathcal{J}}= & \left.F_{1} F_{2}\right|_{\sqrt{-g}}=1+\sqrt{-g}\left[\int \mathrm{d} t \underline{\xi}_{\mathrm{cl}}(t) \cdot \underline{\chi}(t) \underline{\chi}^{2}(t)\right. \\
& \left.+\frac{3}{4} \int \mathrm{d} t \underline{\underline{\chi}}(t) \cdot \underline{\xi}_{\mathrm{cl}}(t)+\frac{N-1}{4} \int \mathrm{d} t \underline{\chi}(t) \cdot \underline{\xi}_{\mathrm{cl}}(t)\right] .
\end{aligned}
$$

Finally, the third way to obtain a correction to the two-point function is via the perturbative subtraction term [the second term in Eq. (3.7)], which involves the perturbative (Gaussian) correlation function $\mathcal{Z}_{0, \alpha_{1} \alpha_{2}}^{(2)}\left(t_{1}, t_{2}\right)$. It is somewhat analogous to the factor $F_{3}$ for the partition function.

(iv) As it will turn out, one can actually show that the two-point Green function $G\left(t_{1}, t_{2}\right)$ is a function of the time difference $t_{1}-t_{2}$. This has consequences for the evaluation of the two-point correlator at zero momentum transfer, as follows. Namely, a priori, one would formulate the Fourier transform of the two-point correlator as follows:

$\mathcal{G}\left(p_{1}, p_{2}\right)=\int \mathrm{d} t_{1} \int \mathrm{d} t_{2} \mathrm{e}^{-\mathrm{i} p_{1} t_{1}-\mathrm{i} p_{2} t_{2}} \mathcal{G}\left(t_{1}, t_{2}\right)$.

Using the property

$$
\mathcal{G}\left(t_{1}, t_{2}\right)=\mathcal{G}\left(t_{1}-t_{2}\right),
$$

one finds

$$
\mathcal{G}\left(p_{1}, p_{2}\right)=2 \pi \delta\left(p_{1}+p_{2}\right) \mathcal{G}\left(p_{1}\right) .
$$

We will be interested here in the two-point correlation function at zero momentum transfer, which, in view of the above considerations, is just the Fourier transform of $G(\tau)$ at zero momentum, i.e., 


$$
\begin{gathered}
\mathcal{G}(p=0)=\int \mathrm{d} \tau \mathcal{G}(\tau), \\
\frac{\partial^{2}}{\partial p^{2}} \mathcal{G}(p=0)=\int \mathrm{d} \tau\left(-\tau^{2}\right) \mathcal{G}(\tau),
\end{gathered}
$$

where the latter expression enters the CallanSymanzik equation.

\section{First correction term}

Let us summarize the formulas mentioned above. We have, for the first term in Eq. (3.15),

$\left[\mathcal{G}_{\alpha \beta}\left(t_{1}, t_{2}\right)\right]_{1}=\frac{\delta_{\alpha \beta}}{N}\left[\mathcal{G}_{\gamma \gamma}\left(t_{1}, t_{2}\right)\right]_{1}=\frac{\delta_{\alpha \beta}}{N}\left[\mathcal{G}\left(t_{1}, t_{2}\right)\right]_{1}$.

For later reference, it is customary to define a recurrent prefactor as

$$
\mathcal{Q}(g)=\frac{1}{\Gamma(N / 2)}\left(-\frac{8}{g}\right)^{N / 2} \exp \left(\frac{4}{3 g}\right)
$$

Corrections to the two-point function can be derived based on Eqs. (3.19) and (3.25), and lead to the formula

$$
\begin{aligned}
\frac{\left[\mathcal{G}\left(t_{1}, t_{2}\right)\right]_{1}}{\mathcal{Q}(g)}= & \int \mathrm{d} t_{0}\left\langle\mathcal{F}_{\mathcal{J}} q_{\gamma}\left(t_{1}-t_{0}\right) q_{\gamma}\left(t_{2}-t_{0}\right)\right\rangle \mathcal{F}_{\mathcal{Z}} \\
\approx & \int \mathrm{d} t_{0}\left\langle q_{\mathrm{cl}, \gamma}\left(t_{1}-t_{0}\right) q_{\mathrm{cl}, \gamma}\left(t_{2}-t_{0}\right)\right\rangle \mathcal{F}_{\mathcal{Z}} \\
& +\int \mathrm{d} t_{0}\left\langle\left(\mathcal{F}_{\mathcal{J}}-1\right) q_{\gamma}\left(t_{1}-t_{0}\right) q_{\gamma}\left(t_{2}-t_{0}\right)\right\rangle,
\end{aligned}
$$

which is valid up to relative order $g$. Here, the path integral expectation value $\langle\cdot\rangle$ has been defined in Eq. (2.44). The first term has the classical field configuration $q_{\mathrm{cl}, \gamma}\left(t_{1}-t_{0}\right)$ and the correction factor $\mathcal{F}_{\mathcal{Z}}$, while the second has the correction factor $\mathcal{F}_{\mathcal{J}}$. We can thus write the two-point correlation function, up to relative order $g$, as follows:

$$
\begin{aligned}
& \frac{\left[\mathcal{G}\left(t_{1}, t_{2}\right)\right]_{1}}{\mathcal{Q}(g)}=\int \mathrm{d} t_{0}\left(-\frac{1}{g}\right) \xi_{\mathrm{cl}}\left(t_{1}-t_{0}\right) \xi_{\mathrm{cl}}\left(t_{2}-t_{0}\right) \mathcal{F}_{\mathcal{Z}}+\int \mathrm{d} t_{0}\left\langle\chi_{\mathrm{L}}\left(t_{1}-t_{0}\right) \chi_{\mathrm{L}}\left(t_{2}-t_{0}\right)+\underline{\chi}_{\mathrm{T}}\left(t_{1}-t_{0}\right) \cdot \underline{\chi}_{\mathrm{T}}\left(t_{2}-t_{0}\right)\right\rangle \\
& +\int \mathrm{d} t_{0}\left\langle\sqrt{-\frac{1}{g}} \chi_{\mathrm{L}}\left(t_{1}-t_{0}\right) \xi_{\mathrm{cl}}\left(t_{2}-t_{0}\right)\left(\mathcal{F}_{\mathcal{J}}-1\right)\right\rangle+\int \mathrm{d} t_{0}\left\langle\sqrt{-\frac{1}{g}} \chi_{\mathrm{L}}\left(t_{2}-t_{0}\right) \xi_{\mathrm{cl}}\left(t_{1}-t_{0}\right)\left(\mathcal{F}_{\mathcal{J}}-1\right)\right\rangle,
\end{aligned}
$$

where the first and the second terms have already been treated. The Wick theorem immediately leads to

$$
\left\langle\chi_{\mathrm{L}}\left(t_{1}-t_{0}\right) \chi_{\mathrm{L}}\left(t_{2}-t_{0}\right)+\underline{\chi}_{\mathrm{T}}\left(t_{1}-t_{0}\right) \cdot \underline{\chi}_{\mathrm{T}}\left(t_{2}-t_{0}\right)\right\rangle=\boldsymbol{\Delta}_{\mathrm{L}}\left(t_{1}-t_{0}, t_{2}-t_{0}\right)+(N-1) \boldsymbol{\Delta}_{\mathrm{T}}\left(t_{1}-t_{0}, t_{2}-t_{0}\right) .
$$

For the third and the fourth terms in Eq. (3.34), one consults the definition of $\mathcal{F}_{\mathcal{J}}$ and applies the Wick theorem in order, to obtain

$$
\begin{aligned}
\frac{\left[\mathcal{G}\left(t_{1}, t_{2}\right)\right]_{1}}{\mathcal{Q}(g)}= & \int \mathrm{d} t_{0}\left\{-\frac{1}{g} \xi_{\mathrm{cl}}\left(t_{1}-t_{0}\right) \xi_{\mathrm{cl}}\left(t_{2}-t_{0}\right) \mathcal{F}_{\mathcal{Z}}+\left[\boldsymbol{\Delta}_{\mathrm{L}}\left(t_{1}-t_{0}, t_{2}-t_{0}\right)+(N-1) \boldsymbol{\Delta}_{\mathrm{T}}\left(t_{1}-t_{0}, t_{2}-t_{0}\right)\right]\right\} \\
& +\left\{\int \mathrm{d} t_{0} \int \mathrm{d} t \xi_{\mathrm{cl}}\left(t_{1}-t_{0}\right) \xi_{\mathrm{cl}}(t)\left[3 \boldsymbol{\Delta}_{\mathrm{L}}\left(t_{2}-t_{0}, t\right) \boldsymbol{\Delta}_{\mathrm{L}}(t, t)+(N-1) \boldsymbol{\Delta}_{\mathrm{L}}\left(t_{2}-t_{0}, t\right) \boldsymbol{\Delta}_{\mathrm{T}}(t, t)\right]\right. \\
& \left.-\frac{3}{4} \int \mathrm{d} t_{0} \int \mathrm{d} t \xi_{\mathrm{cl}}\left(t_{1}-t_{0}\right) \ddot{\xi}_{\mathrm{cl}}(t) \boldsymbol{\Delta}_{\mathrm{L}}\left(t_{2}-t_{0}, t\right)+\frac{N-1}{4} \int \mathrm{d} t_{0} \int \mathrm{d} t \xi_{\mathrm{cl}}\left(t_{1}-t_{0}\right) \xi_{\mathrm{cl}}(t) \boldsymbol{\Delta}_{\mathrm{L}}\left(t_{2}-t_{0}, t\right)\right\} \\
& +\left\{t_{1} \leftrightarrow t_{2}\right\},
\end{aligned}
$$

where $\left\{t_{1} \leftrightarrow t_{2}\right\}$ denotes the terms listed in the previous curly brackets, with the time variables $t_{1}$ and $t_{2}$ interchanged. The first two terms do not involve an additional integration over $t$, because they do not incur corrections from the factor $\mathcal{F}_{\mathcal{J}}$. In the result, we have the integration over $t$, from the product $F_{1} F_{2}$, the integration over $t_{0}$, from the collective coordinate, and the integration over $t^{\prime}$, from the evaluation of the correlation function at zero momentum transfer.
If we shift, in Eq. (3.36), the integration variable, uniformly, according to

$$
t_{0} \rightarrow t_{0}+t_{2}
$$

then we can show the time translation invariance identity [see Eq. (3.27)]

$$
\left[\mathcal{G}\left(t_{1}, t_{2}\right)\right]_{1}=\left[\mathcal{G}\left(t_{1}-t_{2}, 0\right)\right]_{1}=\left[\mathcal{G}\left(t^{\prime}\right)\right]_{1}, t^{\prime}=t_{1}-t_{2} .
$$


However, in the last term in Eq. (3.36) [the one characterized by the replacement $\left(t_{1} \leftrightarrow t_{2}\right)$ ], it is actually advantageous to shift the integration variable according to $t_{0} \rightarrow t_{0}+t_{1}$, at variance with Eq. (3.37).

Eventually, we will need to calculate the integral [see Eq. (3.29)]

$$
\int \mathrm{d} t^{\prime}\left[\mathcal{G}\left(t^{\prime}\right)\right]_{1}=[\mathcal{G}(p=0)]_{1} .
$$

We find

$$
\begin{aligned}
\frac{[\mathcal{G}(p=0)]_{1}}{\mathcal{Q}(g)}= & \int \mathrm{d} t^{\prime} \int \mathrm{d} t_{0}\left\{-\frac{1}{g} \xi_{\mathrm{cl}}\left(t^{\prime}-t_{0}\right) \xi_{\mathrm{cl}}\left(-t_{0}\right) \mathcal{F}_{\mathcal{Z}}+\left[\boldsymbol{\Delta}_{\mathrm{L}}\left(t^{\prime}-t_{0},-t_{0}\right)+(N-1) \boldsymbol{\Delta}_{\mathrm{T}}\left(t^{\prime}-t_{0},-t_{0}\right)\right]\right\} \\
& +2 \int \mathrm{d} t^{\prime} \int \mathrm{d} t_{0} \int \mathrm{d} t\left\{\xi_{\mathrm{cl}}\left(t^{\prime}-t_{0}\right) \xi_{\mathrm{cl}}(t)\left[3 \boldsymbol{\Delta}_{\mathrm{L}}\left(-t_{0}, t\right) \boldsymbol{\Delta}_{\mathrm{L}}(t, t)+(N-1) \boldsymbol{\Delta}_{\mathrm{L}}\left(-t_{0}, t\right) \boldsymbol{\Delta}_{\mathrm{T}}(t, t)\right]\right. \\
& \left.-\frac{3}{4} \xi_{\mathrm{cl}}\left(t^{\prime}-t_{0}\right) \ddot{\xi}_{\mathrm{cl}}(t) \boldsymbol{\Delta}_{\mathrm{L}}\left(-t_{0}, t\right)+\frac{N-1}{4} \xi_{\mathrm{cl}}\left(t^{\prime}-t_{0}\right) \xi_{\mathrm{cl}}(t) \boldsymbol{\Delta}_{\mathrm{L}}\left(-t_{0}, t\right)\right\} .
\end{aligned}
$$

This integral is divergent for large $\beta$, but the infinities are removed upon consideration of the perturbative term $[\mathcal{G}(p=0)]_{2}$, to be considered in the following.

In the last term in Eq. (3.40), we have the integration over $t$, which is the integration variable in the perturbative factor $\mathcal{F}_{\mathcal{J}}$, the integration over the collective coordinate $t_{0}$, and the integration over the time translation variable $t^{\prime}=$ $t_{1}-t_{2}$ of the Green function. The presence of the instanton, which vanishes exponentially for a large argument, guarantees the convergence of the integral.

\section{E. Second correction term}

We now concentrate on the second term in Eq. (3.15),

$$
\left[\mathcal{G}_{\alpha \beta}\left(t_{1}, t_{2}\right)\right]_{2}=-\frac{\mathcal{Z}_{0, \alpha \beta}^{(2)}\left(t_{1}, t_{2}\right) \operatorname{Im} \mathcal{Z}_{1}(\underline{0})}{\mathcal{Z}_{0}^{2}(\underline{0})},
$$

and we can remember that, according to our previous considerations, the discussion can be restricted to the expression

$$
\begin{aligned}
{\left[\mathcal{G}\left(t_{1}, t_{2}\right)\right]_{2} } & =\left[\mathcal{G}_{\gamma \gamma}\left(t_{1}, t_{2}\right)\right]_{2} \\
& =-\frac{\mathcal{Z}_{0, \gamma \gamma}^{(2)}\left(t_{1}, t_{2}\right)}{\mathcal{Z}_{0}(\underline{0})} \frac{\operatorname{Im} \mathcal{Z}_{1}(\underline{0})}{\mathcal{Z}_{0}(\underline{0})} .
\end{aligned}
$$

In comparison to $\left[\mathcal{G}\left(t_{1}, t_{2}\right)\right]_{1}$, the expression $\left[\mathcal{G}\left(t_{1}, t_{2}\right)\right]_{2}$ is of relative order $g$, because it lacks the presence of the classical paths, which are, themselves, of order $\sqrt{-1 / g}$. So, we evaluate the expression (3.41) to leading order only.

We have already anticipated the cancellation mechanism for the $\beta$ parameter; indeed, there is no dependence on $\beta$ in $\mathcal{Z}_{0, \alpha \beta}^{(2)}\left(t_{1}, t_{2}\right)$. However, there is a multiplicative factor $\beta$ in $\operatorname{Im} \mathcal{Z}_{1}$, due to the integral over the collective coordinate $t_{0}$. This factor $\beta$ should cancel against other divergences in $\beta$, to be found in the integrals over the propagators in
$\mathcal{Z}_{1, \alpha \beta}^{(2)}\left(t_{1}, t_{2}\right)$. In fact, we show in the following, that the term $\left[\mathcal{G}\left(t_{1}, t_{2}\right)\right]_{2}$ exactly furnishes the terms necessary for the removal of the infinities in Eq. (3.40).

First, we have, upon perturbative expansion,

$$
\frac{\mathcal{Z}_{0, \gamma \gamma}^{(2)}\left(t_{1}, t_{2}\right)}{\mathcal{Z}_{0}(\underline{0})}=\left\langle q_{\gamma}\left(t_{1}\right) q_{\gamma}\left(t_{2}\right)\right\rangle_{0}=N \boldsymbol{\Delta}_{0}\left(t_{1}, t_{2}\right),
$$

where the integration measure $\langle\cdot\rangle_{0}$ has been defined in Eq. (2.31). Compared to the instanton partition function $\mathcal{Z}_{1}(\beta)$, the generating function $\mathcal{Z}_{1}(\underline{J})$ lacks the integration over the end point $q_{0}$, while the same is true for the perturbative contributions $\mathcal{Z}_{0}(\beta)$ versus $\mathcal{Z}_{0}(\underline{J})$. However, the lacking integration cancels in the ratio, and we can write, with Eq. (2.36),

$$
\begin{aligned}
\frac{\operatorname{Im} \mathcal{Z}_{1}(\underline{0})}{\mathcal{Z}_{0}(\underline{0})}= & \frac{\operatorname{Im} \mathcal{Z}_{1}(\beta)}{\mathcal{Z}_{0}(\beta)}=\frac{\beta}{\Gamma(N / 2)}\left(-\frac{8}{g}\right)^{N / 2} \exp \left(\frac{4}{3 g}\right) \\
& \times\left[1+g\left(\frac{7}{32} N^{2}+\frac{9}{16} N+\frac{5}{24}\right)\right] .
\end{aligned}
$$

So, we finally get

$$
\begin{aligned}
{\left[\mathcal{G}\left(t_{1}, t_{2}\right)\right]_{2}=} & -\frac{1}{\Gamma(N / 2)}\left(-\frac{8}{g}\right)^{N / 2} \exp \left(\frac{4}{3 g}\right) \\
& \times\left[N \beta \Delta_{0}\left(t_{1}, t_{2}\right)\right] .
\end{aligned}
$$

Here, according to Eq. (2.24b), the free propagator $\boldsymbol{\Delta}_{0}\left(t_{1}, t_{2}\right)$ has the translation invariance property

$$
\boldsymbol{\Delta}_{0}\left(t_{1}, t_{2}\right)=\boldsymbol{\Delta}_{0}\left(t_{1}-t_{2}\right)=\boldsymbol{\Delta}_{0}\left(t_{1}-t_{2}, 0\right) .
$$

For the purposes of the removal of the infinities discussed in Sec. III E, we can reformulate the Fourier transform of this expression at zero momentum as follows: 


$$
\begin{aligned}
{[\mathcal{G}(p=0)]_{2}=} & -\frac{1}{\Gamma(N / 2)}\left(-\frac{8}{g}\right)^{N / 2} \exp \left(\frac{4}{3 g}\right) \\
& \times \int \mathrm{d} t_{0} \int \mathrm{d} t^{\prime}\left[\boldsymbol{\Delta}_{0}\left(0, t^{\prime}\right)+(N-1) \boldsymbol{\Delta}_{0}\left(0, t^{\prime}\right)\right],
\end{aligned}
$$

where $t^{\prime}=t_{1}-t_{2}$. We have replaced $\beta \rightarrow \int \mathrm{d} t_{0}$. We recall that the integration limits in all given integrals cover the range $-\infty<\tau<\infty$ for all Euclidean time parameters $\tau$, unless indicated otherwise; however, these limits are incurred in terms of the limiting process $-\beta / 2<\tau<\beta / 2$, where we let $\beta \rightarrow \infty$.

\section{F. Evaluation of the corrections}

We add the expressions from Eqs. (3.40) and (3.47) and consider the sum of $[\mathcal{G}(p=0)]_{1}$ and $[\mathcal{G}(p=0)]_{2}$. The substitutions $t^{\prime} \rightarrow t^{\prime}+t_{0}$ and subsequently $t_{0} \rightarrow-t_{0}$ serve to simplify the expressions (the Jacobian in each case is unity). One can finally write $\mathcal{G}(p=0)$ as

$$
\frac{\mathcal{G}(p=0)}{\mathcal{Q}(g)}=\sum_{i=1}^{7} \mathcal{R}_{i},
$$

where the $\mathcal{R}_{i}$ terms $(i=1, \ldots, 7)$ are defined in the following (see also Fig. 3). For the evaluation of the expression of $\mathcal{R}_{1}$, we refer to integral $H_{1}$ listed in Appendix A, and write (a)

(b)

(c)

(d)

(e)
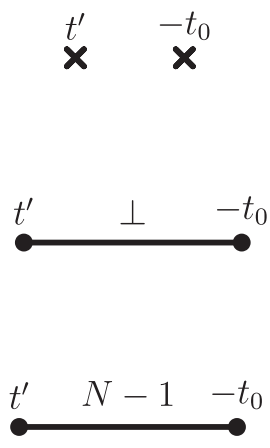

$$
-2 \pi^{2}\left(\frac{7}{32} N^{2}+\frac{9}{16} N+\frac{5}{24}\right)
$$

$-4$

$$
(N-1)\left(1-\frac{\pi^{2}}{4}-\frac{7}{2} \zeta(3)\right)
$$

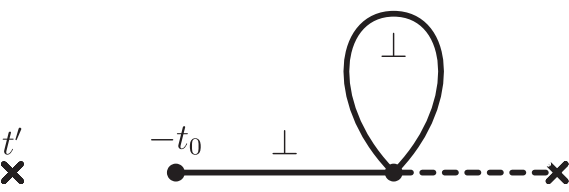

$-\frac{3 \pi^{2}}{2}$

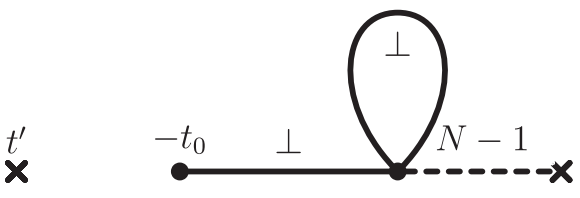

$-\frac{\pi^{2}}{4}(N-1)$
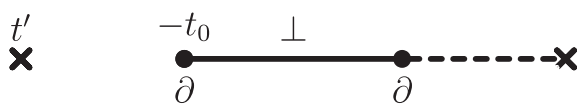

$-\frac{3 \pi^{2}}{2}$
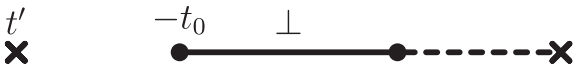

0

FIG. 3. Diagrammatic representation of the seven two-loop corrections to the two-point function at zero momentum, for a onedimensional $\phi^{4}$ theory with an $O(N)$ internal symmetry. The contribution of the diagrams is written beside each contribution. The total result of order $g$ is of the form given in Eq. (3.53). One of the classical field configurations, associated with the time variable $t^{\prime}$ (or $t^{\prime}-t_{0}$, before a suitable change of variable), is somehow "detached" from the rest of the diagram. Recall that the transverse character of the propagator (excluding the instanton configurations) is denoted by the symbol $\perp$, and that the variable $t^{\prime}=t_{1}-t_{2}$ enters in view of the time translation invariance of the Green function. Incidentally, the diagrams for the second derivative of the two-point function (Sec. III

$\mathrm{G}$ ) and for the four-point function (Sec. III H) are the same as those depicted here, with (in the case of the four-point function) two more detached instantons. 


$$
\begin{aligned}
\mathcal{R}_{1} & =-\frac{1}{g}\left[\int \mathrm{d} t^{\prime} \xi_{\mathrm{cl}}\left(t^{\prime}\right)\right]^{2} \mathcal{F}_{\mathcal{Z}}=-\frac{1}{g}\left(H_{1}\right)^{2} \mathcal{F}_{Z} \\
& =-\frac{2 \pi^{2}}{g}-2 \pi^{2}\left(\frac{7}{32} N^{2}+\frac{9}{16} N+\frac{5}{24}\right),
\end{aligned}
$$

where we use Eq. (3.19). For the term $\mathcal{R}_{2}$, one has

$$
\mathcal{R}_{2}=\int \mathrm{d} t^{\prime} \int \mathrm{d} t_{0}\left[\boldsymbol{\Delta}_{\mathrm{L}}\left(t^{\prime}, t_{0}\right)-\boldsymbol{\Delta}_{0}\left(t^{\prime}, t_{0}\right)\right]=I_{1}=-4 .
$$

For the integral $I_{1}$, we again refer to Appendix A. The integral $\mathcal{R}_{3}$ involves the transverse propagator,

$$
\begin{aligned}
\mathcal{R}_{3} & =(N-1) \int \mathrm{d} t^{\prime} \int \mathrm{d} t_{0}\left[\boldsymbol{\Delta}_{\mathrm{T}}\left(t^{\prime}, t_{0}\right)-\boldsymbol{\Delta}_{0}\left(t^{\prime}, t_{0}\right)\right] \\
& =(N-1) I_{2}=(N-1)\left[1-\frac{\pi^{2}}{4}-\frac{7}{2} \zeta(3)\right]
\end{aligned}
$$

(see also Appendix A). The rest of the terms are

$$
\begin{aligned}
\mathcal{R}_{4} & =6 \int \mathrm{d} t^{\prime} \xi_{\mathrm{cl}}\left(t^{\prime}\right) \int \mathrm{d} t \xi_{\mathrm{cl}}(t) \Delta_{\mathrm{L}}(t, t) \int \mathrm{d} t_{0} \Delta_{\mathrm{L}}\left(t, t_{0}\right) \\
& =6 H_{1} J_{3}=\frac{3 \pi^{2}}{2}, \\
\mathcal{R}_{5} & =2(N-1) \int \mathrm{d} t^{\prime} \xi_{\mathrm{cl}}\left(t^{\prime}\right) \int \mathrm{d} t_{\mathrm{cl}}(t) \Delta_{\mathrm{T}}(t, t) \int \mathrm{d} t_{0} \Delta_{\mathrm{L}}\left(t, t_{0}\right) \\
& =2(N-1) H_{1} J_{4}=\frac{\pi^{2}}{4}(N-1), \\
\mathcal{R}_{6} & =-\frac{3}{2} \int \mathrm{d} t^{\prime} \xi_{\mathrm{cl}}\left(t^{\prime}\right) \int \mathrm{d} \ddot{\xi}_{\mathrm{cl}}(t) \int \mathrm{d} t_{0} \Delta_{\mathrm{L}}\left(t, t_{0}\right) \\
& =-\frac{3}{2} H_{1} J_{2}=-\frac{3 \pi^{2}}{2}, \\
\mathcal{R}_{7} & =\frac{N-1}{2} \int \mathrm{d} t^{\prime} \xi_{\mathrm{cl}}\left(t^{\prime}\right) \int \mathrm{d} t \xi_{\mathrm{cl}}(t) \int \mathrm{d} t_{0} \Delta_{\mathrm{L}}\left(t, t_{0}\right) \\
& =\frac{N-1}{2} H_{1} J_{1}=0 .
\end{aligned}
$$

The integrals $J_{i}(i=1, \ldots, 4)$ are listed in Appendix A. The end result is

$$
\begin{aligned}
\frac{\mathcal{G}(p=0)}{\mathcal{Q}(g)}= & -\frac{2 \pi^{2}}{g}+\left(\frac{7}{2} \zeta(3)-5-\frac{5 \pi^{2}}{12}\right) \\
& \times\left[1-\frac{9 \pi^{2}}{8}-\frac{7}{2} \zeta(3)\right] N-\frac{7 \pi^{2}}{16} N^{2} .
\end{aligned}
$$

It is interesting to note that, in the limit $N \rightarrow \infty$, the leading contribution to the coefficient of relative order $g$ comes from the partition function correction $\mathcal{F}_{\mathcal{Z}}$. Furthermore, in the limit of small $g$, the imaginary part of the Green function described by $\mathcal{G}(p=0)$ is positive.

\section{G. Second derivative of the correlator}

To evaluate the second derivative of the two-point correlation function, we recall Eq. (3.40), subtract the perturbative term with subscript "2," and insert a factor $\left(-t^{\prime 2}\right)$ in the $t^{\prime}$ integration,

$$
\frac{\frac{\partial^{2}}{\partial p^{2}} \mathcal{G}(p=0)}{\mathcal{Q}(g)}=\sum_{i=1}^{7} \mathcal{S}_{i} .
$$

After appropriate substitutions in the integration variables, we obtain the following expression, the following integrals are generated, after obvious symmetry considerations,

$$
\begin{aligned}
\mathcal{S}_{1} & =\frac{2}{g} \int \mathrm{d} t^{\prime} t^{\prime 2} \xi_{\mathrm{cl}}\left(t^{\prime}\right) \int \mathrm{d} t_{0} \xi_{\mathrm{cl}}\left(t_{0}\right) \mathcal{F}_{\mathcal{Z}}=\frac{2}{g} H_{3} H_{1} \mathcal{F}_{\mathcal{Z}} \\
& =\frac{\pi^{4}}{g}+\pi^{4}\left(\frac{7}{32} N^{2}+\frac{9}{16} N+\frac{5}{24}\right) .
\end{aligned}
$$

The subtracted propagators, with the momentum derivative insertion, give rise to the following expressions:

$$
\begin{aligned}
\mathcal{S}_{2} & =\int \mathrm{d} t^{\prime} \int \mathrm{d} t_{0}\left[-\left(t^{\prime}-t_{0}\right)^{2}\right]\left[\boldsymbol{\Delta}_{\mathrm{L}}\left(t^{\prime}, t_{0}\right)-\boldsymbol{\Delta}_{0}\left(t^{\prime}, t_{0}\right)\right] \\
& =-\bar{K}_{1}=-2-\frac{\pi^{2}}{2}-21 \zeta(3), \\
\mathcal{S}_{3}= & (N-1) \int \mathrm{d} t^{\prime} \int \mathrm{d} t_{0}\left[-\left(t^{\prime}-t_{0}\right)^{2}\right] \\
& \times\left[\boldsymbol{\Delta}_{\mathrm{T}}\left(t^{\prime}, t_{0}\right)-\boldsymbol{\Delta}_{0}\left(t^{\prime}, t_{0}\right)\right] \\
= & -(N-1) \bar{K}_{2}=(N-1)\left(-6+\frac{\pi^{4}}{8}+\frac{93}{2} \zeta(5)\right) .
\end{aligned}
$$

The $\bar{K}$ integrals are listed in Appendix A. The rest of the terms involve instanton configurations. The first of these is

$$
\begin{aligned}
\mathcal{S}_{4}= & 6 \int \mathrm{d} t^{\prime} \int \mathrm{d} t_{0} \int \mathrm{d} t\left[-\left(t^{\prime}-t_{0}\right)^{2}\right] \xi_{\mathrm{cl}}\left(t^{\prime}\right) \xi_{\mathrm{cl}}(t) \\
& \times \boldsymbol{\Delta}_{\mathrm{L}}\left(t_{0}, t\right) \boldsymbol{\Delta}_{\mathrm{L}}(t, t) \\
= & -6 H_{3} J_{3}-6 H_{1} L_{3}=+\frac{\pi^{2}}{2}-\frac{9 \pi^{4}}{4} .
\end{aligned}
$$

The term with combined transverse and longitudinal propagators is

$$
\begin{aligned}
\mathcal{S}_{5}= & 2(N-1) \int \mathrm{d} t^{\prime} \int \mathrm{d} t_{0} \int \mathrm{d} t\left[-\left(t^{\prime}-t_{0}\right)^{2}\right] \xi_{\mathrm{cl}}\left(t^{\prime}\right) \xi_{\mathrm{cl}}(t) \\
& \times \Delta_{\mathrm{L}}\left(t_{0}, t\right) \boldsymbol{\Delta}_{\mathrm{T}}(t, t) \\
= & -2(N-1)\left(H_{3} J_{4}+H_{1} L_{4}\right)=-\frac{5 \pi^{4}}{8}(N-1) .
\end{aligned}
$$


The term with the second derivative of the instanton is

$$
\begin{aligned}
\mathcal{S}_{6} & =\frac{3}{2} \int \mathrm{d} t^{\prime} \int \mathrm{d} t_{0} \int \mathrm{d} t\left(t^{\prime}-t_{0}\right)^{2} \xi_{\mathrm{cl}}\left(t^{\prime}\right) \ddot{\xi}_{\mathrm{cl}}(t) \Delta_{\mathrm{L}}\left(t_{0}, t\right) \\
& =\frac{3}{2}\left(H_{3} J_{2}+H_{1} L_{2}\right)=\frac{3 \pi^{4}}{2} .
\end{aligned}
$$

The last term generated by the Jacobian factor $\mathcal{F}_{\mathcal{J}}$ is

$$
\begin{aligned}
\mathcal{S}_{7} & =-\frac{N-1}{2} \int \mathrm{d} t^{\prime} \int \mathrm{d} t_{0} \int \mathrm{d} t\left(t^{\prime}-t_{0}\right)^{2} \xi_{\mathrm{cl}}\left(t^{\prime}\right) \xi_{\mathrm{cl}}(t) \Delta_{\mathrm{L}}\left(t_{0}, t\right) \\
& =-\frac{N-1}{2}\left(H_{3} J_{1}+H_{1} L_{1}\right)=-\frac{\pi^{4}}{4}(N-1)
\end{aligned}
$$

The overall result is

$$
\begin{aligned}
\frac{\frac{\partial^{2}}{\partial p^{2}} \mathcal{G}(p=0)}{\mathcal{Q}(g)}= & \frac{\pi^{4}}{g}+\frac{5 \pi^{4}}{24}+4-21 \zeta(3)-\frac{93}{2} \zeta(5) \\
& +N\left(-\frac{3 \pi^{4}}{16}-6+\frac{93}{2} \zeta(5)\right)+\frac{7 \pi^{4}}{32} N^{2} .
\end{aligned}
$$

Again, it is somewhat surprising that the leading term for large $N$ comes from the correction factor $\mathcal{F}_{\mathcal{Z}}$.

\section{H. Four-point correlation function}

We are interested here in understanding the imaginary part of the four-point correlation function to relative order $g$. To this end, it is first of all necessary to remember that we only need to consider the first three terms $\mathcal{K}_{1,2,3}$ on the right-hand side of Eq. (3.8), because the remaining terms are of relative order $g^{2}$. The dominant term, for small $g$, is given by $\mathcal{K}_{1}$. We write, in analogy to Eq. (3.15), $\mathcal{G}_{\alpha \beta \gamma \delta}$ as the sum of two terms, the first of which is dominating,

$$
\begin{aligned}
\mathcal{G}_{\alpha \beta \gamma \delta}\left(t_{1}, t_{2}, t_{3}, t_{4}\right)= & \operatorname{Im} \mathcal{W}_{1, \alpha \beta \gamma \delta}^{(4)}\left(t_{1}, t_{2}, t_{3}, t_{4}\right) \\
= & {\left[\mathcal{G}_{\alpha \beta \gamma \delta}\left(t_{1}, t_{2}, t_{3}, t_{4}\right)\right]_{1} } \\
& +\left[\mathcal{G}_{\alpha \beta \gamma \delta}\left(t_{1}, t_{2}, t_{3}, t_{4}\right)\right]_{2} \\
\approx & {\left[\mathcal{G}_{\alpha \beta \gamma \delta}\left(t_{1}, t_{2}, t_{3}, t_{4}\right)\right]_{1} . }
\end{aligned}
$$

The leading term is

$$
\left[\mathcal{G}_{\alpha \beta \gamma \delta}\left(t_{1}, t_{2}, t_{3}, t_{4}\right)\right]_{1}=\frac{\operatorname{Im} \mathcal{Z}_{1, \alpha \beta \gamma \delta}^{(4)}\left(t_{1}, t_{2}, t_{3}, t_{4}\right)}{\mathcal{Z}_{0}(\underline{0})} .
$$

The additional perturbative term, which cancels a few divergences, is

$$
\begin{aligned}
{\left[\mathcal{G}_{\alpha \beta \gamma \delta}\left(t_{1}, t_{2}, t_{3}, t_{4}\right)\right]_{2}=} & -\frac{\mathcal{Z}_{0, \alpha \beta}^{(2)}\left(t_{1}, t_{2}\right) \operatorname{Im} \mathcal{Z}_{1, \gamma \delta}^{(2)}\left(t_{3}, t_{4}\right)}{\mathcal{Z}_{0}^{2}(\underline{0})}-\frac{\mathcal{Z}_{0, \alpha \gamma}^{(2)}\left(t_{1}, t_{3}\right) \operatorname{Im} \mathcal{Z}_{1, \beta \delta}^{(2)}\left(t_{2}, t_{4}\right)}{\mathcal{Z}_{0}^{2}(\underline{0})}-\frac{\mathcal{Z}_{0, \alpha \delta}^{(2)}\left(t_{1}, t_{4}\right) \operatorname{Im} \mathcal{Z}_{1, \beta \gamma}^{(2)}\left(t_{2}, t_{3}\right)}{\mathcal{Z}_{0}^{2}(\underline{0})} \\
& -\frac{\mathcal{Z}_{0, \gamma \delta}^{(2)}\left(t_{3}, t_{4}\right) \operatorname{Im} \mathcal{Z}_{1, \alpha \beta}^{(2)}\left(t_{1}, t_{2}\right)}{\mathcal{Z}_{0}^{2}(\underline{0})}-\frac{\mathcal{Z}_{0, \beta \delta}^{(2)}\left(t_{2}, t_{4}\right) \operatorname{Im} \mathcal{Z}_{1, \alpha \gamma}^{(2)}\left(t_{1}, t_{4}\right)}{\mathcal{Z}_{0}^{2}(\underline{0})}-\frac{\mathcal{Z}_{0, \beta \gamma}^{(2)}\left(t_{2}, t_{3}\right) \operatorname{Im} \mathcal{Z}_{1, \alpha \delta}^{(2)}\left(t_{1}, t_{3}\right)}{\mathcal{Z}_{0}^{2}(\underline{0})} .
\end{aligned}
$$

Just as with the two-point function, the additional compensating perturbative terms cancel certain divergences from diagrams that originate from the leading term, in the sense of the replacement

$$
\boldsymbol{\Delta}_{\mathrm{L}}\left(t^{\prime}-t_{0}, t_{0}\right) \rightarrow \boldsymbol{\Delta}_{\mathrm{L}}\left(t^{\prime}-t_{0}, t_{0}\right)-\boldsymbol{\Delta}_{0}\left(t^{\prime}-t_{0}, t_{0}\right),
$$

$$
\boldsymbol{\Delta}_{\mathrm{T}}\left(t^{\prime}-t_{0}, t_{0}\right) \rightarrow \boldsymbol{\Delta}_{\mathrm{T}}\left(t^{\prime}-t_{0}, t_{0}\right)-\boldsymbol{\Delta}_{0}\left(t^{\prime}-t_{0}, t_{0}\right) .
$$

The leading expression for the four-point function is easily derived, based on the same reasoning as was used in Eq. (3.15),

$$
\begin{aligned}
\mathcal{G}_{\alpha \beta \gamma \delta}\left(t_{1}, t_{2}, t_{3}, t_{4}\right) & \approx \frac{1}{g^{2}}\left\langle u_{\alpha} u_{\beta} u_{\gamma} u_{\delta}\right\rangle_{S_{N-1}} \int \mathrm{d} t_{0} \xi_{\mathrm{cl}}\left(t_{1}-t_{0}\right) \xi_{\mathrm{cl}}\left(t_{2}-t_{0}\right) \xi_{\mathrm{cl}}\left(t_{3}-t_{0}\right) \xi_{\mathrm{cl}}\left(t_{4}-t_{0}\right) \\
& =\mathcal{Q}(g) \frac{\delta_{\alpha \beta} \delta_{\gamma \delta}+\delta_{\alpha \gamma} \delta_{\beta \delta}+\delta_{\alpha \delta} \delta_{\gamma \beta}}{N(N+2)} \frac{1}{g^{2}} J\left(t_{1}, t_{2}, t_{3}, t_{4}\right)
\end{aligned}
$$

where $J\left(t_{1}, t_{2}, t_{3}, t_{4}\right)$ reads as

$$
\begin{aligned}
J\left(t_{1}, t_{2}, t_{3}, t_{4}\right)= & \int \mathrm{d} t_{0} \xi_{\mathrm{cl}}\left(t_{1}-t_{0}\right) \xi_{\mathrm{cl}}\left(t_{2}-t_{0}\right) \xi_{\mathrm{cl}}\left(t_{3}-t_{0}\right) \xi_{\mathrm{cl}}\left(t_{4}-t_{0}\right) \\
= & -\frac{8\left(t_{1}-t_{4}\right)}{\sinh \left(t_{1}-t_{2}\right) \sinh \left(t_{1}-t_{3}\right) \sinh \left(t_{1}-t_{4}\right)}-\frac{8\left(t_{2}-t_{4}\right)}{\sinh \left(t_{2}-t_{1}\right) \sinh \left(t_{2}-t_{3}\right) \sinh \left(t_{2}-t_{4}\right)} \\
& -\frac{8\left(t_{3}-t_{4}\right)}{\sinh \left(t_{3}-t_{1}\right) \sinh \left(t_{3}-t_{2}\right) \sinh \left(t_{3}-t_{4}\right)}
\end{aligned}
$$


This formula might seem "asymmetric" as the time coordinate $t_{4}$ has been singled out. However, a closer inspection shows that the formula actually is symmetric with respect to a cyclic permutation of the time coordinates $t_{i}$ (with $\left.i=1,2,3,4\right)$.

According to Eqs. (3.22) and (3.23), we can define, for the two-point function, a "scalar" (with respect to the internal symmetry group) quantity $\mathcal{G}$, which is obtained from $\mathcal{G}_{\alpha \beta}$, via division by the factor $\delta_{\alpha \beta} / N$. The same is true for the four-point function, where we first note that $\mathcal{G}_{\alpha \beta \gamma \delta}$ can be written as

$$
\begin{aligned}
\mathcal{G}_{\alpha \beta \gamma \delta}\left(t_{1}, t_{2}, t_{3}, t_{4}\right)= & \frac{\delta_{\alpha \beta} \delta_{\gamma \delta}+\delta_{\alpha \gamma} \delta_{\beta \delta}+\delta_{\alpha \delta} \delta_{\gamma \beta}}{N(N+2)} \\
& \times \mathbf{G}\left(t_{1}, t_{2}, t_{3}, t_{4}\right) .
\end{aligned}
$$

In leading order, one has

$$
\mathbf{G}\left(t_{1}, t_{2}, t_{3}, t_{4}\right) \approx \mathcal{Q}(g) \frac{1}{g^{2}} J\left(t_{1}, t_{2}, t_{3}, t_{4}\right) .
$$

In turn, $\mathbf{G}\left(t_{1}, t_{2}, t_{3}, t_{4}\right)$ can be extracted from $\mathcal{G}_{\alpha \beta \gamma \delta}\left(t_{1}, t_{2}, t_{3}, t_{4}\right)$ as

$$
\delta_{\alpha \beta} \delta_{\gamma \delta} \mathcal{G}_{\alpha \beta \gamma \delta}\left(t_{1}, t_{2}, t_{3}, t_{4}\right)=\mathbf{G}\left(t_{1}, t_{2}, t_{3}, t_{4}\right)
$$

Now, one can show that $\mathbf{G}\left(t_{1}, t_{2}, t_{3}, t_{4}\right)$ can be written as a function of the differences of the time coordinates only,

$$
\mathbf{G}\left(t_{1}, t_{2}, t_{3}, t_{4}\right)=\mathbf{G}\left(t_{1}-t_{4}, t_{2}-t_{4}, t_{3}-t_{4}\right) .
$$

For the leading term, given in Eq. (3.69), this relationship can be checked by inspection. Let us investigate the Fourier transform

$$
\begin{aligned}
\mathbf{G}\left(p_{1}, p_{2}, p_{3}, p_{4}\right)= & \int \mathrm{d} t_{1} \int \mathrm{d} t_{2} \int \mathrm{d} t_{3} \int \mathrm{d} t_{4} \\
& \times \mathrm{e}^{-\mathrm{i}\left(p_{1} t_{1}+p_{2} t_{2}+p_{3} t_{3}+p_{4} t_{4}\right)} \\
& \times \mathbf{G}\left(t_{1}-t_{4}, t_{2}-t_{4}, t_{3}-t_{4}\right) .
\end{aligned}
$$

By a suitable change of variable, one can show that

$$
\begin{aligned}
\mathbf{G}\left(p_{1}, p_{2}, p_{3}, p_{4}\right)= & 2 \pi \delta\left(p_{1}+p_{2}+p_{3}+p_{4}\right) \mathbf{G}\left(p_{1}, p_{2}, p_{3}\right) \\
= & 2 \pi \delta\left(p_{1}+p_{2}+p_{3}+p_{4}\right) \\
& \times \int \mathrm{d} t^{\prime} \int \mathrm{d} t^{\prime \prime} \int \mathrm{d} t^{\prime \prime \prime} \\
& \times \mathrm{e}^{-\mathrm{i}\left(p_{1} t^{\prime}+p_{2} t^{\prime \prime}+p_{3} t^{\prime \prime \prime}\right)} \mathbf{G}\left(t^{\prime}, t^{\prime \prime}, t^{\prime \prime \prime}\right) .
\end{aligned}
$$

Our task will be focused on

$$
\begin{aligned}
\mathbf{G} & \left(p_{1}=0, p_{2}=0, p_{3}=0\right) \\
& =\mathbf{G}\left(p_{i=1,2,3}=0\right) \\
& =\int \mathrm{d} t^{\prime} \int \mathrm{d} t^{\prime \prime} \int \mathrm{d} t^{\prime \prime \prime} \mathbf{G}\left(t^{\prime}, t^{\prime \prime}, t^{\prime \prime \prime}\right) .
\end{aligned}
$$

Note that the integral $J$ defined in Eq. (3.69) can be written as

$J\left(t_{1}, t_{2}, t_{3}, t_{4}\right)=J\left(t_{1}-t_{4}, t_{2}-t_{4}, t_{3}-t_{4}\right)$,

$$
\begin{aligned}
J\left(t^{\prime}, t^{\prime \prime}, t^{\prime \prime \prime}\right)= & -\frac{8 t^{\prime}}{\sinh \left(t^{\prime}-t^{\prime \prime}\right) \sinh \left(t^{\prime}-t^{\prime \prime \prime}\right) \sinh \left(t^{\prime}\right)} \\
& -\frac{8 t^{\prime \prime}}{\sinh \left(t^{\prime \prime}-t^{\prime}\right) \sinh \left(t^{\prime \prime}-t^{\prime \prime \prime}\right) \sinh \left(t^{\prime \prime}\right)} \\
& -\frac{8 t^{\prime \prime \prime}}{\sinh \left(t^{\prime \prime \prime}-t^{\prime}\right) \sinh \left(t^{\prime \prime \prime}-t^{\prime \prime}\right) \sinh \left(t^{\prime \prime \prime}\right)},
\end{aligned}
$$

so that, in leading order,

$$
\begin{aligned}
\frac{\mathbf{G}\left(p_{i=1,2,3}=0\right)}{\mathcal{Q}(g)} & \approx \frac{1}{g^{2}} \int \mathrm{d} t^{\prime} \int \mathrm{d} t^{\prime \prime} \int \mathrm{d} t^{\prime \prime \prime} J\left(t^{\prime}, t^{\prime \prime}, t^{\prime \prime \prime}\right) \\
& =\frac{H_{1}^{4}}{g^{2}}=\frac{4 \pi^{4}}{g^{2}} .
\end{aligned}
$$

It is clear that we have the same structure of corrections as for the two-point function. One can conveniently express the correction as the sum of seven terms, which contribute up to relative order $g$,

$$
\frac{\mathbf{G}\left(p_{i=1,2,3}=0\right)}{\mathcal{Q}(g)}=\sum_{i=1}^{7} \mathcal{T}_{i},
$$

where the $\mathcal{T}_{i}$ will be defined in the following. We have the first correction $\mathcal{T}_{1}$ "for free," because it is just the multiplicative correction $\mathcal{F}_{Z}$, multiplying the leading-order instanton result,

$$
\mathcal{T}_{1}=\frac{4 \pi^{4}}{g^{2}} \mathcal{F}_{\mathcal{Z}}=\frac{4 \pi^{4}}{g^{2}}+\frac{\pi^{4}}{g}\left(\frac{5}{6}+\frac{9}{4} N+\frac{7}{8} N^{2}\right) .
$$

For the Green function in coordinate space, the replacement of two classical fields in the leading term $\operatorname{Im} \mathcal{Z}_{1, \alpha \beta \gamma \delta}^{(4)}\left(t_{1}, t_{2}, t_{3}, t_{4}\right)$ by two fluctuations leads to two terms $\left[\mathcal{T}_{2}\right]_{1}$ and $\left[\mathcal{T}_{3}\right]_{1}$, where the subscript $[\cdots]_{1}$ is motivated by Eq. (3.64). After suitable variables change, their contribution to the four-point function at zero momentum can be written as

$$
\begin{aligned}
{\left[\mathcal{T}_{2}\right]_{1}=} & -\frac{6}{g} \int \mathrm{d} t_{0} \int \mathrm{d} t^{\prime} \int \mathrm{d} t^{\prime \prime} \int \mathrm{d} t^{\prime \prime \prime} \\
& \times \xi_{\mathrm{cl}}\left(t_{0}\right) \xi_{\mathrm{cl}}\left(t^{\prime \prime \prime}\right) \Delta_{\mathrm{L}}\left(t^{\prime}, t^{\prime \prime}\right),
\end{aligned}
$$




$$
\begin{aligned}
{\left[\mathcal{T}_{3}\right]_{1}=} & -\frac{2(N-1)}{g} \int \mathrm{d} t_{0} \int \mathrm{d} t^{\prime} \int \mathrm{d} t^{\prime \prime} \int \mathrm{d} t^{\prime \prime \prime} \\
& \times \xi_{\mathrm{cl}}\left(t_{0}\right) \xi_{\mathrm{cl}}\left(t^{\prime \prime \prime}\right) \Delta_{\mathrm{T}}\left(t^{\prime}, t^{\prime \prime}\right) .
\end{aligned}
$$

To evaluate the compensating perturbative terms $\left[\mathcal{T}_{2}\right]_{2}$ and $\left[\mathcal{T}_{3}\right]_{2}$ from Eq. (3.65), one replaces [see Eq. (3.43)]

$$
\frac{\mathcal{Z}_{0, \alpha \beta}^{(2)}\left(t_{1}, t_{2}\right)}{\mathcal{Z}_{0}(\underline{0})} \rightarrow \frac{\delta_{\alpha \beta}}{N}\left[N \boldsymbol{\Delta}_{0}\left(t_{1}, t_{2}\right)\right] .
$$

(There is no integration over a collective coordinate here, as we are analyzing the perturbative propagator.) For the term involving the instanton saddle point, we have

$$
\begin{aligned}
\frac{\operatorname{Im} \mathcal{Z}_{1, \gamma \delta}^{(2)}\left(t_{3}, t_{4}\right)}{\mathcal{Z}_{0}(\underline{0})} & \rightarrow\left(-\frac{1}{g}\right) \frac{\delta_{\gamma \delta}}{N} \mathcal{Q}(g) \\
& \times \int \mathrm{d} t_{0} \xi_{\mathrm{cl}}\left(t_{3}-t_{0}\right) \xi_{\mathrm{cl}}\left(t_{4}-t_{0}\right) .
\end{aligned}
$$

After suitable variable changes, one arrives at the following "counterterm" from the expression in Eq. (3.65),

$$
\begin{aligned}
{\left[\mathcal{T}_{2}\right]_{2}=} & \frac{6}{g} \int \mathrm{d} t_{0} \int \mathrm{d} t^{\prime} \int \mathrm{d} t^{\prime \prime} \int \mathrm{d} t^{\prime \prime \prime} \\
& \times \xi_{\mathrm{cl}}\left(t_{0}\right) \xi_{\mathrm{cl}}\left(t^{\prime \prime \prime}\right) \Delta_{0}\left(t^{\prime}, t^{\prime \prime}\right), \\
{\left[\mathcal{T}_{3}\right]_{2}=} & \frac{2(N-1)}{g} \int \mathrm{d} t_{0} \int \mathrm{d} t^{\prime} \int \mathrm{d} t^{\prime \prime} \int \mathrm{d} t^{\prime \prime \prime} \\
& \times \xi_{\mathrm{cl}}\left(t_{0}\right) \xi_{\mathrm{cl}}\left(t^{\prime \prime \prime}\right) \Delta_{0}\left(t^{\prime}, t^{\prime \prime}\right) .
\end{aligned}
$$

Hence, $\mathcal{T}_{2}$ and $\mathcal{T}_{3}$ can be expressed as

$$
\begin{aligned}
& \mathcal{T}_{2}=\left[\mathcal{T}_{2}\right]_{1}+\left[\mathcal{T}_{2}\right]_{2}=-\frac{6}{g} H_{1}^{2} I_{1}=\frac{48 \pi^{2}}{g}, \\
& \mathcal{T}_{3}=\left[\mathcal{T}_{3}\right]_{1}+\left[\mathcal{T}_{3}\right]_{2}=\frac{N-1}{g}\left(\pi^{4}-4 \pi^{2}+14 \pi^{2} \zeta(3)\right) .
\end{aligned}
$$

Note that $\mathcal{T}_{2}$ and $\mathcal{T}_{3}$ are analogous to the terms $G_{2}$ and $G_{3}$, incurred for the two-point function. The integrals $H_{1}, I_{1}$, and $I_{2}$ can be found in Appendix A.

Now we must treat the analogues of the terms $G_{4,5,6,7}$, generated by the Jacobian factor $\mathcal{F}_{\mathcal{J}}$, originally derived for the two-point function. These terms are generated by the replacement of one classical field configuration by a fluctuation combined, via the Wick theorem, with a contraction with a second fluctuation in the action + Jacobian factor $\mathcal{F}_{\mathcal{J}}$. For the two-point function, we have two possibilities to choose one field out of two for the cases where only one field is replaced by a fluctuation. For the four-point function, we have four such possibilities, and so we could tentatively conjecture that the corrections $\mathcal{T}_{4}, \mathcal{T}_{5}$,
$\mathcal{T}_{6}$, and $\mathcal{T}_{7}$ receive a relative factor of 2 , as compared to the two-point Green function. This will turn out to be a good guess, but it needs to be verified by an explicit calculation.

We now consider the term

$$
\sqrt{-g} \int \mathrm{d} t \underline{\xi}_{\mathrm{cl}}(t) \cdot \underline{\chi}(t) \underline{\chi}^{2}(t)
$$

from Eq. (3.25). One obtains two corrections $\mathcal{T}_{4}$ and $\mathcal{T}_{5}$ to $\mathbf{G}\left(p_{i=1,2,3}=0\right) / \mathcal{Q}(g)$, according to Eq. (3.79),

$$
\begin{aligned}
\mathcal{T}_{4}= & -\frac{12}{g} \int \mathrm{d} t_{0} \int \mathrm{d} t^{\prime} \int \mathrm{d} t^{\prime \prime} \int \mathrm{d} t^{\prime \prime \prime} \int \mathrm{d} t \xi_{\mathrm{cl}}\left(t_{0}\right) \xi_{\mathrm{cl}}\left(t^{\prime \prime \prime}\right) \\
& \times \xi_{\mathrm{cl}}\left(t^{\prime}\right) \xi_{\mathrm{cl}}(t) \Delta_{\mathrm{L}}(t, t) \Delta_{\mathrm{L}}\left(t, t^{\prime \prime}\right) \\
= & -\frac{12}{g} H_{1}^{3} J_{3}=-\frac{6 \pi^{4}}{g} \\
\mathcal{T}_{5}= & -\frac{4(N-1)}{g} \int \mathrm{d} t_{0} \int \mathrm{d} t^{\prime} \int \mathrm{d} t^{\prime \prime} \int \mathrm{d} t^{\prime \prime \prime} \int \mathrm{d} t \\
& \times \xi_{\mathrm{cl}}\left(t_{0}\right) \xi_{\mathrm{cl}}\left(t^{\prime \prime \prime}\right) \xi_{\mathrm{cl}}\left(t^{\prime}\right) \xi_{\mathrm{cl}}(t) \Delta_{\mathrm{T}}(t, t) \Delta_{\mathrm{L}}\left(t, t^{\prime \prime}\right) \\
= & -\frac{4(N-1)}{g} H_{1}^{3} J_{4}=-\frac{\pi^{4}}{g}(N-1) .
\end{aligned}
$$

From the term

$$
\sqrt{-g}\left[-\frac{3}{4} \int \mathrm{d} t \underline{\chi}(t) \cdot \underline{\xi}_{\mathrm{cl}}(t)\right]
$$

in Eq. (3.25), one has the correction

$$
\begin{aligned}
\mathcal{T}_{6}= & \frac{3}{g} \int \mathrm{d} t_{0} \int \mathrm{d} t^{\prime} \int \mathrm{d} t^{\prime \prime} \int \mathrm{d} t^{\prime \prime \prime} \int \mathrm{d} t \\
& \times \xi_{\mathrm{cl}}\left(t_{0}\right) \xi_{\mathrm{cl}}\left(t^{\prime \prime \prime}\right) \xi_{\mathrm{cl}}\left(t^{\prime}\right) \ddot{\xi}_{\mathrm{cl}}(t) \Delta_{\mathrm{L}}\left(t, t^{\prime \prime}\right) \\
= & \frac{3}{g} H_{1}^{3} J_{2}=\frac{6 \pi^{4}}{g} .
\end{aligned}
$$

The last correction is from the term

$$
\sqrt{-g} \frac{N-1}{4} \int \mathrm{d} t \underline{\chi}(t) \cdot \underline{\xi}_{\mathrm{cl}}(t)
$$

in Eq. (3.25) and reads as

$$
\begin{aligned}
\mathcal{T}_{7}= & -\frac{N-1}{g} \int \mathrm{d} t_{0} \int \mathrm{d} t^{\prime} \int \mathrm{d} t^{\prime \prime} \int \mathrm{d} t^{\prime \prime \prime} \\
& \times \int \mathrm{d} t \xi_{\mathrm{cl}}\left(t_{0}\right) \xi_{\mathrm{cl}}\left(t^{\prime \prime \prime}\right) \xi_{\mathrm{cl}}\left(t^{\prime}\right) \xi_{\mathrm{cl}}(t) \Delta_{\mathrm{L}}\left(t, t^{\prime \prime}\right) \\
= & \frac{N-1}{g} H_{1}^{3} J_{1}=0 .
\end{aligned}
$$

The end result is given as follows: 


$$
\begin{aligned}
\frac{\mathbf{G}\left(p_{i=1,2,3}=0\right)}{\mathcal{Q}(g)}= & \sum_{i=1}^{7} \mathcal{T}_{i} \\
= & \frac{4 \pi^{4}}{g^{2}}+\frac{\pi^{2}}{g}\left[52+\frac{5 \pi^{2}}{6}-14 \zeta(3)\right. \\
& \left.+N\left(\frac{9 \pi^{2}}{4}-4+14 \zeta(3)\right)+\frac{7 \pi^{2}}{8} N^{2}\right],
\end{aligned}
$$

and involves, again, a couple of Riemann zeta functions. Finally, we recall the Fourier transform of Eq. (3.70) in the form

$$
\begin{aligned}
\frac{\mathcal{G}_{\alpha \beta \gamma \delta}\left(p_{i=1,2,3}=0\right)}{\mathcal{Q}(g)}= & \frac{\delta_{\alpha \beta} \delta_{\gamma \delta}+\delta_{\alpha \gamma} \delta_{\beta \delta}+\delta_{\alpha \delta} \delta_{\gamma \beta}}{N(N+2)} \\
& \times \frac{\mathbf{G}\left(p_{i=1,2,3}=0\right)}{\mathcal{Q}(g)}
\end{aligned}
$$

exhibiting the angular structure within the internal $O(N)$ group.

\section{Two-point wigglet insertion}

In this section we will derive the large-order behavior of the two-point correlation function with a wigglet insertion computed at zero momentum. Using the previous results relative to the two- and four-point functions, its derivation will be straightforward. We define the (imaginary part of the) wigglet insertion into the two-point Green function $\mathcal{G}_{\alpha \beta}\left(t_{1}, t_{2}\right)$, according to Eq. (3.7), as follows:

$$
\begin{aligned}
\mathcal{G}_{\alpha \beta}^{(1,2)}\left(t_{1}, t_{2}\right) & =\left.\frac{\partial^{2}}{\partial m^{2}} \mathcal{G}_{\alpha \beta}\left(m, t_{1}, t_{2}\right)\right|_{m^{2}=1} \\
& =\left.\frac{\partial^{2}}{\partial m^{2}} \operatorname{Im} \mathcal{W}_{1, \alpha \beta}^{(2)}\left(m, t_{1}, t_{2}\right)\right|_{m^{2}=1} \\
& =-\frac{1}{2} \int \mathrm{d} s \operatorname{Im} \mathcal{W}_{\alpha \beta \gamma \gamma}^{(4)}\left(s, s, t_{1}, t_{2}\right) \\
& =-\frac{1}{2} \int \mathrm{d} s \mathcal{G}_{\alpha \beta \gamma \gamma}\left(s, s, t_{1}, t_{2}\right) .
\end{aligned}
$$

Here, $\mathcal{G}_{\alpha \beta}\left(m, t_{1}, t_{2}\right)$ is the analogue of $\mathcal{G}_{\alpha \beta}\left(t_{1}, t_{2}\right)$, defined in Eq. (3.15), but with respect to an action with a variable mass term,

$$
\mathcal{S}[m, q(t)]=\int \mathrm{d} t\left(\frac{1}{2} \dot{q}^{2}+\frac{m^{2}}{2} q^{2}+\frac{1}{4} g q^{4}\right)
$$

In view of the result (3.68) for the leading contribution to the four-point function, we have the relation [see Eq. (3.68)]

$$
\begin{aligned}
\mathcal{G}_{\alpha \beta}^{(1,2)}\left(t_{1}, t_{2}\right) & =-\frac{1}{2} \int \mathrm{d} s \mathcal{G}_{\alpha \beta \gamma \gamma}\left(s, s, t_{1}, t_{2}\right) \\
& =-\frac{\mathcal{Q}(g)}{2} \frac{\delta_{\alpha \beta}}{N} \frac{1}{g^{2}} \frac{16\left(t_{1}-t_{2}\right)}{\sinh \left(t_{1}-t_{2}\right)},
\end{aligned}
$$

where we have used Eq. (3.32). One can show that $\mathcal{G}_{\alpha \beta}^{(1,2)}\left(t_{1}, t_{2}\right)$ can be written in terms of the time coordinate differences only,

$$
\mathcal{G}_{\alpha \beta}^{(1,2)}\left(t_{1}, t_{2}\right)=\mathcal{G}_{\alpha \beta}^{(1,2)}\left(t_{1}-t_{2}\right) .
$$

In leading order, we anticipate the result

$$
\begin{aligned}
\int \mathrm{d}\left(t_{1}-t_{2}\right) \mathcal{G}_{\alpha \beta}^{(1,2)}\left(t_{1}-t_{2}\right) & \approx-\frac{\mathcal{Q}(g)}{2} \frac{\delta_{\alpha \beta}}{N} \frac{H_{1}^{2} H_{2}}{g^{2}} \\
& =-\frac{\mathcal{Q}(g)}{2} \frac{\delta_{\alpha \beta}}{N} \frac{8 \pi^{2}}{g^{2}}
\end{aligned}
$$

where we have used, again, integrals from Appendix A. To analyze the two-loop corrections, we first need to remember the angular structure. First, it is easy to see that, from Eq. (3.70), we have

$$
\mathcal{G}_{\alpha \beta \gamma \gamma}\left(t_{1}, t_{2}, t_{3}, t_{4}\right)=\frac{\delta_{\alpha \beta}}{N} \mathbf{G}\left(t_{1}, t_{2}, t_{3}, t_{4}\right) .
$$

So,

$$
\begin{aligned}
\mathcal{G}_{\alpha \beta}^{(1,2)}\left(t_{1}, t_{2}\right) & =-\frac{\delta_{\alpha \beta}}{2 N} \int \mathrm{d} s \mathbf{G}\left(s, s, t_{1}-t_{2}\right) \\
& =-\frac{\delta_{\alpha \beta}}{2 N} \mathbf{H}\left(t_{1}, t_{2}\right)=-\frac{\delta_{\alpha \beta}}{2 N} \mathbf{H}\left(t_{1}-t_{2}\right),
\end{aligned}
$$

where we appeal to Eq. (3.73) and implicitly define the function $\mathbf{H}\left(t_{1}, t_{2}\right)=\mathbf{H}\left(t_{1}-t_{2}\right)$. Let us investigate the Fourier transform

$$
\begin{aligned}
\mathbf{H}\left(p_{1}, p_{2}\right) & =\int \mathrm{d} t_{1} \int \mathrm{d} t_{2} \mathrm{e}^{-\mathrm{i}\left(p_{1} t_{1}+p_{2} t_{2}\right)} \int \mathrm{d} s \mathbf{H}\left(t_{1}, t_{2}\right) \\
& =2 \pi \delta\left(p_{1}+p_{2}\right) \mathbf{H}\left(p_{1}\right),
\end{aligned}
$$

where

$$
\mathbf{H}(p)=\int \mathrm{d} t^{\prime} \int \mathrm{d} t^{\prime \prime} \mathrm{e}^{-\mathrm{i} p t^{\prime \prime}} \mathbf{G}\left(t^{\prime}, t^{\prime}, t^{\prime \prime}\right),
$$

so that

$$
\mathbf{H}(p=0)=\int \mathrm{d} s \int \mathrm{d}\left(t_{1}-t_{2}\right) \mathbf{G}\left(s, s, t_{1}, t_{2}\right),
$$

where we again use Eq. (3.73). In leading order, in view of Eqs. (3.71) and (3.77b), one has 


$$
\begin{aligned}
\frac{\mathbf{H}(p=0)}{\mathcal{Q}(g)} & \approx \frac{1}{g^{2}} \int \mathrm{d} t^{\prime} \int \mathrm{d} t^{\prime \prime} J\left(t^{\prime}, t^{\prime}, t^{\prime \prime}\right) \\
& =\frac{H_{1}^{2} H_{2}}{g^{2}}=\frac{8 \pi^{2}}{g^{2}}
\end{aligned}
$$

confirming the leading-order result given in Eq. (3.101). One can conveniently express the correction as the sum of seven terms, which contribute up to relative order $g$,

$$
\frac{\mathbf{H}(p=0)}{\mathcal{Q}(g)}=\sum_{i=1}^{7} \mathcal{U}_{i}
$$

We have the first correction "for free," because it is just the multiplicative correction $\mathcal{F}_{Z}$, multiplying the leading-order instanton result,

$\mathcal{U}_{1}=\frac{8 \pi^{2}}{g^{2}} \mathcal{F}_{\mathcal{Z}}=\frac{8 \pi^{2}}{g^{2}}+\frac{2 \pi^{2}}{g}\left(\frac{5}{6}+\frac{9}{4} N+\frac{7}{8} N^{2}\right)$.

Just as we did in our analysis of the four-point function, we now replace, in the Green function in coordinate space, two classical fields by fluctuations and obtain two terms,

$$
\begin{aligned}
{\left[\mathcal{U}_{2}\right]_{1}=} & -\frac{1}{g} \int \mathrm{d} t^{\prime} \int \mathrm{d} t^{\prime \prime} \Delta_{\mathrm{L}}\left(t^{\prime}, t^{\prime \prime}\right) \int \mathrm{d} t^{\prime \prime \prime}\left[\xi_{\mathrm{cl}}\left(t^{\prime \prime \prime}\right)\right]^{2} \\
& -\frac{4}{g} \int \mathrm{d} t^{\prime} \xi_{\mathrm{cl}}\left(t^{\prime}\right) \int \mathrm{d} t^{\prime \prime} \Delta_{\mathrm{L}}\left(t^{\prime}, t^{\prime \prime}\right) \int \mathrm{d} t^{\prime \prime \prime} \xi_{\mathrm{cl}}\left(t^{\prime \prime \prime}\right) \\
& -\frac{1}{g} \int \mathrm{d} t^{\prime} \Delta_{\mathrm{L}}\left(t^{\prime}, t^{\prime}\right) \int \mathrm{d} t^{\prime \prime} \xi_{\mathrm{cl}}\left(t^{\prime \prime}\right) \int \mathrm{d} t^{\prime \prime \prime} \xi_{\mathrm{cl}}\left(t^{\prime \prime \prime}\right), \\
{\left[\mathcal{U}_{3}\right]_{1}=} & -\frac{N-1}{g} \int \mathrm{d} t^{\prime} \int \mathrm{d} t^{\prime \prime} \Delta_{\mathrm{T}}\left(t^{\prime}, t^{\prime \prime}\right) \int \mathrm{d} t^{\prime \prime \prime}\left[\xi_{\mathrm{cl}}\left(t^{\prime \prime \prime}\right)\right]^{2} \\
& -\frac{N-1}{g} \int \mathrm{d} t^{\prime} \Delta_{\mathrm{T}}\left(t^{\prime}, t^{\prime}\right) \\
& \times \int \mathrm{d} t^{\prime \prime} \xi_{\mathrm{cl}}\left(t^{\prime \prime}\right) \int \mathrm{d} t^{\prime \prime \prime} \xi_{\mathrm{cl}}\left(t^{\prime \prime \prime}\right) .
\end{aligned}
$$

From the perturbative compensating term in Eq. (3.65), we have

$$
\begin{aligned}
{\left[\mathcal{U}_{2}\right]_{2}=} & \frac{1}{g} \int \mathrm{d} t^{\prime} \int \mathrm{d} t^{\prime \prime} \Delta_{0}\left(t^{\prime}, t^{\prime \prime}\right) \int \mathrm{d} t^{\prime \prime \prime}\left[\xi_{\mathrm{cl}}\left(t^{\prime \prime \prime}\right)\right]^{2} \\
& +\frac{4}{g} \int \mathrm{d} t^{\prime} \xi_{\mathrm{cl}}\left(t^{\prime}\right) \int \mathrm{d} t^{\prime \prime} \Delta_{0}\left(t^{\prime}, t^{\prime \prime}\right) \int \mathrm{d} t^{\prime \prime \prime} \xi_{\mathrm{cl}}\left(t^{\prime \prime \prime}\right) \\
& -\frac{1}{g} \int \mathrm{d} t^{\prime} \Delta_{0}\left(t^{\prime}, t^{\prime}\right) \int \mathrm{d} t^{\prime \prime} \xi_{\mathrm{cl}}\left(t^{\prime \prime}\right) \int \mathrm{d} t^{\prime \prime \prime} \xi_{\mathrm{cl}}\left(t^{\prime \prime \prime}\right),
\end{aligned}
$$

$$
\begin{aligned}
{\left[\mathcal{U}_{3}\right]_{2}=} & \frac{N-1}{g} \int \mathrm{d} t^{\prime} \int \mathrm{d} t^{\prime \prime} \Delta_{0}\left(t^{\prime}, t^{\prime \prime}\right) \int \mathrm{d} t^{\prime \prime \prime}\left[\xi_{\mathrm{cl}}\left(t^{\prime \prime \prime}\right)\right]^{2} \\
& +\frac{N-1}{g} \int \mathrm{d} t^{\prime} \Delta_{0}\left(t^{\prime}, t^{\prime}\right) \\
& \times \int \mathrm{d} t^{\prime \prime} \xi_{\mathrm{cl}}\left(t^{\prime \prime}\right) \int \mathrm{d} t^{\prime \prime \prime} \xi_{\mathrm{cl}}\left(t^{\prime \prime \prime}\right)
\end{aligned}
$$

With the help of the integrals listed in Appendix A, we can express $\mathcal{U}_{2}$ and $\mathcal{U}_{3}$ as follows:

$$
\begin{aligned}
\mathcal{U}_{2} & =-\frac{1}{g} H_{2} I_{1}-\frac{4}{g} H_{1}\left(J_{1}-J_{1}^{(0)}\right)-\frac{1}{g} H_{1}^{2} N_{1} \\
& =\frac{48+31 \pi^{2}}{3 g} \\
\mathcal{U}_{3} & =-\frac{N-1}{g} H_{2} I_{2}-\frac{N-1}{g} H_{1}^{2} N_{2} \\
& =\frac{2(N-1)}{g}\left[\pi^{2}-2+7 \zeta(3)\right] .
\end{aligned}
$$

We now consider the term

$$
\sqrt{-g}\left[\int \mathrm{d} t \underline{\xi}_{\mathrm{cl}}(t) \cdot \underline{\chi}(t) \underline{\chi}^{2}(t)\right]
$$

in the Jacobian Eq. (3.25). After the application of the Wick theorem and suitable variable changes, one arrives at a contribution to $\mathbf{H}(p=0) / \mathcal{Q}(g)$, consisting of the sum of two terms, $\mathcal{U}_{4}$ and $\mathcal{U}_{5}$,

$$
\begin{aligned}
\mathcal{U}_{4}= & -\frac{6}{g} \int \mathrm{d} t \xi_{\mathrm{cl}}(t) \Delta_{\mathrm{L}}(t, t) \\
& \times \int \mathrm{d} t^{\prime} \Delta_{\mathrm{L}}\left(t, t^{\prime}\right) \int \mathrm{d} t^{\prime \prime}\left[\xi_{\mathrm{cl}}\left(t^{\prime \prime}\right)\right] \int \mathrm{d} t^{\prime \prime \prime}\left[\xi_{\mathrm{cl}}\left(t^{\prime \prime \prime}\right)\right]^{2} \\
& -\frac{6}{g} \int \mathrm{d} t \xi_{\mathrm{cl}}(t) \Delta_{\mathrm{L}}(t, t) \int \mathrm{d} t^{\prime} \xi_{\mathrm{cl}}\left(t^{\prime}\right) \Delta_{\mathrm{L}}\left(t, t^{\prime}\right)\left[\int \mathrm{d} t^{\prime \prime} \xi_{\mathrm{cl}}\left(t^{\prime \prime}\right)\right]^{2} \\
= & -\frac{6}{g} H_{1} H_{2} J_{3}-\frac{6}{g} H_{1}^{2} M_{3}=-\frac{19 \pi^{2}}{3 g}, \\
\mathcal{U}_{5}= & -\frac{2(N-1)}{g} \int \mathrm{d} t \xi_{\mathrm{cl}}(t) \Delta_{\mathrm{T}}(t, t) \int \mathrm{d} t^{\prime} \Delta_{\mathrm{L}}\left(t, t^{\prime}\right) \\
& \times \int \mathrm{d} t^{\prime \prime}\left[\xi_{\mathrm{cl}}\left(t^{\prime \prime}\right)\right] \int \mathrm{d} t^{\prime \prime \prime}\left[\xi_{\mathrm{cl}}\left(t^{\prime \prime \prime}\right)\right]^{2} \\
& -\frac{2(N-1)}{g} \int \mathrm{d} t \xi_{\mathrm{cl}}(t) \Delta_{\mathrm{T}}(t, t) \int \mathrm{d} t^{\prime} \xi_{\mathrm{cl}}\left(t^{\prime}\right) \Delta_{\mathrm{L}}\left(t, t^{\prime}\right) \\
& \times\left[\int \mathrm{d} t^{\prime \prime} \xi_{\mathrm{cl}}\left(t^{\prime \prime}\right)\right]^{2} \\
= & -\frac{2(N-1)}{g} H_{1} H_{2} J_{4}-\frac{2(N-1)}{g} H_{1}^{2} M_{4}=0 .
\end{aligned}
$$

From the term 


$$
\sqrt{-g}\left[-\frac{3}{4} \int \mathrm{d} t \underline{\chi}(t) \cdot \underline{\boldsymbol{\xi}}_{\mathrm{cl}}(t)\right]
$$

in the Jacobian given in Eq. (3.25), one has

$$
\begin{aligned}
\mathcal{U}_{6} & =\frac{3}{2 g} \int \mathrm{d} t \xi_{\mathrm{cl}}(t) \int \mathrm{d} t^{\prime} \Delta_{\mathrm{L}}\left(t, t^{\prime}\right) \int \mathrm{d} t^{\prime \prime} \xi_{\mathrm{cl}}\left(t^{\prime \prime}\right) \int \mathrm{d} t^{\prime \prime \prime}\left[\xi_{\mathrm{cl}}\left(t^{\prime \prime \prime}\right)\right]^{2} \\
& +\frac{3}{2 g} \int \mathrm{d} t \xi_{\mathrm{cl}}(t) \int \mathrm{d} t^{\prime} \xi_{\mathrm{cl}}\left(t^{\prime}\right) \Delta_{\mathrm{L}}\left(t, t^{\prime}\right)\left[\int \mathrm{d} t^{\prime \prime} \xi_{\mathrm{cl}}\left(t^{\prime \prime}\right)\right]^{2} \\
& =\frac{3}{2 g} H_{1} H_{2} J_{2}+\frac{3}{2 g} H_{1}^{2} M_{2}=\frac{9 \pi^{2}}{g} .
\end{aligned}
$$

The last correction is from the term

$$
\sqrt{-g} \frac{N-1}{4} \int \mathrm{d} t \underline{\chi}(t) \cdot \underline{\xi}_{\mathrm{cl}}(t)
$$

in Eq. (3.25) and leads to the correction

$$
\begin{aligned}
\mathcal{U}_{7}= & -\frac{N-1}{2 g} \int \mathrm{d} t \xi_{\mathrm{cl}}(t) \int \mathrm{d} t^{\prime} \Delta_{\mathrm{L}}\left(t, t^{\prime}\right) \\
& \times \int \mathrm{d} t^{\prime \prime} \xi_{\mathrm{cl}}\left(t^{\prime \prime}\right) \int \mathrm{d} t^{\prime \prime \prime}\left[\xi_{\mathrm{cl}}\left(t^{\prime \prime \prime}\right)\right]^{2} \\
& -\frac{N-1}{2 g} \int \mathrm{d} t \xi_{\mathrm{cl}}(t) \int \mathrm{d} t^{\prime} \xi_{\mathrm{cl}}\left(t^{\prime}\right) \Delta_{\mathrm{L}}\left(t, t^{\prime}\right)\left[\int \mathrm{d} t^{\prime \prime} \xi_{\mathrm{cl}}\left(t^{\prime \prime}\right)\right]^{2} \\
= & -\frac{N-1}{2 g} H_{1} H_{2} J_{1}-\frac{N-1}{2 g} H_{1}^{2} M_{1}=\frac{\pi^{2}(N-1)}{g} .
\end{aligned}
$$

The final result [see Eq. (3.79)] for order- $g$ corrections to the imaginary part of the wigglet insertion into the twopoint function reads as follows:

$$
\begin{aligned}
\frac{\mathbf{H}(p=0)}{\mathcal{Q}(g)} & =\sum_{i=1}^{7} \mathcal{U}_{i}=\frac{8 \pi^{2}}{g^{2}}+\frac{1}{g}\left[20+\frac{35 \pi^{2}}{3}-14 \zeta(3)\right. \\
& \left.+N\left(\frac{15 \pi^{2}}{2}-4+14 \zeta(3)\right)+\frac{7 \pi^{2}}{4} N^{2}\right]
\end{aligned}
$$

It has the same structure as the result for the four-point function, given in Eq. (3.96).

\section{CONCLUSIONS}

\section{A. Large-order behavior: A summary}

In this article, we have concentrated on the onedimensional field theory, with an internal $O(N)$ symmetry group, in the normalization [see Eq. (2.1)]

$$
\mathcal{S}[\underline{q}(t)]=\int \mathrm{d} t\left[\frac{1}{2}\left(\frac{\partial \underline{q}(t)}{\partial t}\right)^{2}+\frac{1}{2} \underline{q}^{2}(t)+\frac{g}{4} \underline{q}^{4}(t)\right],
$$

where $\underline{q}(t)=\left\{q_{1}(t), \ldots, q_{N}(t)\right\}=\sum_{\alpha=1}^{N} q_{\alpha}(t) \underline{e}_{\alpha} . \quad$ The start time $t_{0}$ of the instanton and the coordinate $\tau_{i}$ with $i=1, \ldots, N-1$ are the collective coordinates of the problem. Furthermore, we have analyzed the functional determinant of the transformation of the path integral, into integrals over the collective coordinates and path integrals over paths orthogonal to the zero modes, in Sec. II C, with the result for the Jacobian given in Eq. (2.21).

Based on the results given in Sec. III and the dispersion relations studied in Sec. IB, we are now in the position to write down the large-order behavior of various perturbative expansions, of the partition function and various correlation functions [in the sense of Eqs. (1.8) and (1.9)]. We have from Eq. (2.55), for the imaginary part of the ground-state energy of the $O(N)$ oscillator,

$$
\begin{aligned}
\frac{\operatorname{Im} E_{0}(g)}{\mathcal{Q}(g)} & =\lim _{\beta \rightarrow \infty}\left(-\frac{1}{\mathcal{Q}(g) \beta} \frac{\operatorname{Im} \mathcal{Z}_{1}(\beta)}{\mathcal{Z}_{0}(\beta)}\right) \\
& =-\left[1+g\left(\frac{7}{32} N^{2}+\frac{9}{16} N+\frac{5}{24}\right)\right] .
\end{aligned}
$$

Here, according to Eq. (3.32), one has

$$
\mathcal{Q}(g)=\frac{1}{\Gamma(N / 2)}\left(-\frac{8}{g}\right)^{N / 2} \exp \left(\frac{4}{3 g}\right)
$$

Based on the formalism outlined in Sec. IB, this can be converted to the asymptotics of the perturbative coefficients of the ground-state energy in large orders [see Eq. (2.55)]. One can identify $E_{0}(g) \equiv \mathrm{G}^{(0)}(g)$ with the ground-state energy and study the perturbative expansion $\mathbf{G}^{(0)}(g)=$ $\sum_{K} \mathrm{G}_{K}^{(0)} g^{K}$. Within the conventions outlined in Eq. (1.9), one has $n=0, D=1$ and

$$
\begin{aligned}
& c^{(0)}(N, 1)=-\frac{8^{N / 2}}{\Gamma(N / 2)}, \\
& d^{(0)}(N, 1)=\frac{7}{32} N^{2}+\frac{9}{16} N+\frac{5}{24} .
\end{aligned}
$$

The two-point function has the angular structure [see Eqs. (3.22) and (3.27)]

$$
\operatorname{ImG}_{\alpha \beta}^{(2)}\left(t_{1}-t_{2}\right)=\mathcal{G}_{\alpha \beta}\left(t_{1}, t_{2}\right)=\frac{\delta_{\alpha \beta}}{N} \mathcal{G}\left(t_{1}-t_{2}\right)
$$

We recall that $\mathcal{G}_{\alpha \beta}\left(t_{1}, t_{2}\right)$, as defined in Eq. (3.15), is the imaginary part of the two-point function $\mathrm{G}_{\alpha \beta}\left(t_{1}-t_{2}\right)$, in the sense in Sec. I B. The result for $\mathcal{G}(p=0)=\int \mathrm{d} t^{\prime} \mathcal{G}\left(t^{\prime}\right)$ of the "scalar" two-point function at $p=0$ has been recorded in Eq. (3.53), 


$$
\begin{aligned}
\frac{\mathcal{G}(p=0)}{\mathcal{Q}(g)}= & -\frac{2 \pi^{2}}{g}\left[1+g\left\{\frac{5}{24}+\frac{5}{2 \pi^{2}}-\frac{7 \zeta(3)}{4 \pi^{2}}\right.\right. \\
& \left.\left.+\left(\frac{9}{16}-\frac{1}{2 \pi^{2}}+\frac{7 \zeta(3)}{4 \pi^{2}}\right) N+\frac{7}{32} N^{2}\right\}\right] .
\end{aligned}
$$

For the imaginary part $\mathcal{G}(p=0)$, this translates into the following coefficients in the large-order asymptotics, according to Eq. (1.9):

$$
\begin{aligned}
c^{(2)}(N, 1)= & 2 \pi^{2} \frac{8^{N / 2}}{\Gamma(N / 2)}, \\
d^{(2)}(N, 1)= & \frac{5}{24}+\frac{5}{2 \pi^{2}}-\frac{7 \zeta(3)}{4 \pi^{2}} \\
& +\left(\frac{9}{16}-\frac{1}{2 \pi^{2}}+\frac{7 \zeta(3)}{4 \pi^{2}}\right) N+\frac{7}{32} N^{2} .
\end{aligned}
$$

Of course, we have $n=2$ in the sense of Eq. (1.9) for the two-point function at zero momentum transfer as well as for its derivative, which gives rise to the following imaginary part, according to Eq. (3.62),

$$
\begin{aligned}
\frac{\left.\frac{\partial^{2}}{\partial p^{2}} \mathcal{G}\right|_{p=0}}{\mathcal{Q}(g)}= & \frac{\pi^{4}}{g}\left[1+g\left\{\frac{5}{24}+\frac{4}{\pi^{4}}-\frac{21 \zeta(3)}{\pi^{4}}\right.\right. \\
& \left.\left.-\frac{93 \zeta(5)}{2 \pi^{4}}+\left(-\frac{3}{16}-\frac{6}{\pi^{4}}+\frac{93 \zeta(5)}{2 \pi^{4}}\right) N+\frac{7 N^{2}}{32}\right\}\right] .
\end{aligned}
$$

The leading and subleading large-order asymptotics for the perturbative coefficients of $\left.\frac{\partial^{2}}{\partial p^{2}} \mathcal{G}\right|_{p=0}$ are given as follows [see Eq. (1.9)]:

$$
\begin{aligned}
c^{(\partial p)}(N, 1)= & -\pi^{4} \frac{8^{N / 2}}{\Gamma(N / 2)}, \\
d^{(\partial p)}(N, 1)= & \frac{5}{24}+\frac{4}{\pi^{4}}-\frac{21 \zeta(3)}{\pi^{4}}-\frac{93 \zeta(5)}{2 \pi^{4}} \\
& +\left(-\frac{3}{16}-\frac{6}{\pi^{4}}+\frac{93 \zeta(5)}{2 \pi^{4}}\right) N+\frac{7}{32} N^{2} .
\end{aligned}
$$

For the imaginary part of the four-point function, according to Eq. (3.70), we have

$$
\begin{aligned}
\mathcal{G}_{\alpha \beta \gamma \delta}\left(t_{1}, t_{2}, t_{3}, t_{4}\right)= & \frac{\delta_{\alpha \beta} \delta_{\gamma \delta}+\delta_{\alpha \gamma} \delta_{\beta \delta}+\delta_{\alpha \delta} \delta_{\gamma \beta}}{N(N+2)} \\
& \times \mathbf{G}\left(t_{1}-t_{4}, t_{2}-t_{4}, t_{3}-t_{4}\right) .
\end{aligned}
$$

One defines according to Eq. (3.76),

$$
\begin{aligned}
& \mathbf{G}\left(p_{1}=0, p_{2}=0, p_{3}=0\right)=\mathbf{G}\left(p_{i=1,2,3}=0\right) \\
& \quad=\int \mathrm{d} t^{\prime} \int \mathrm{d} t^{\prime \prime} \int \mathrm{d} t^{\prime \prime \prime} \mathbf{G}\left(t^{\prime}, t^{\prime \prime}, t^{\prime \prime \prime}\right) .
\end{aligned}
$$

The result, to relative order $g$, is given by [according to Eq. (3.96)],

$$
\begin{aligned}
\frac{\mathbf{G}\left(p_{i=1,2,3}=0\right)}{\mathcal{Q}(g)}= & \frac{4 \pi^{4}}{g^{2}}\left[1+g\left\{\frac{5}{24}+\frac{13}{\pi^{2}}-\frac{7 \zeta(3)}{2 \pi^{2}}\right.\right. \\
& \left.\left.+\left(\frac{9}{16}-\frac{1}{\pi^{2}}+\frac{7 \zeta(3)}{2 \pi^{2}}\right) N+\frac{7 N^{2}}{32}\right\}\right] .
\end{aligned}
$$

The perturbative expansion of the imaginary part $\mathbf{G}\left(p_{i=1,2,3}=0\right)$ of the four-point function therefore has the following asymptotics of the perturbative coefficients [see Eq. (1.9) with $n=4$ ]:

$$
\begin{aligned}
c^{(4)}(N, 1)= & 4 \pi^{4} \frac{8^{N / 2}}{\Gamma(N / 2)}, \\
d^{(4)}(N, 1)= & \frac{5}{24}+\frac{13}{\pi^{2}}-\frac{7 \zeta(3)}{2 \pi^{2}} \\
& +\left(\frac{9}{16}-\frac{1}{\pi^{2}}+\frac{7 \zeta(3)}{2 \pi^{2}}\right) N+\frac{7}{32} N^{2} .
\end{aligned}
$$

Finally, for the imaginary part of the two-point function with a wigglet insertion, computed at zero momentum, we have the following expression, according to Eq. (3.103),

$$
\mathcal{G}_{\alpha \beta}^{(1,2)}\left(t_{1}, t_{2}\right)=-\frac{\delta_{\alpha \beta}}{2 N} \mathbf{H}\left(t_{1}-t_{2}\right) .
$$

The quantity of interest is [see Eq. (3.106)]

$$
\mathbf{H}(p=0)=\int \mathrm{d} t^{\prime} \mathbf{H}\left(t^{\prime}\right)
$$

for which we obtain the result [see Eq. (3.123)]

$$
\begin{aligned}
\frac{\mathbf{H}(p=0)}{\mathcal{Q}(g)}= & \frac{8 \pi^{2}}{g^{2}}\left[1+g\left\{\frac{35}{24}+\frac{5}{2 \pi^{2}}-\frac{7 \zeta(3)}{4 \pi^{2}}\right.\right. \\
& \left.\left.+\left(\frac{15}{16}-\frac{1}{2 \pi^{2}}+\frac{7 \zeta(3)}{4 \pi^{2}}\right) N+\frac{7}{32} N^{2}\right\}\right] .
\end{aligned}
$$

The large-order asymptotics of the perturbative coefficients for $\mathbf{H}\left(t_{1}-t_{2}\right)$ are as follows:

$$
\begin{aligned}
c^{(1,2)}(N, 1)= & 8 \pi^{2} \frac{8^{N / 2}}{\Gamma(N / 2)}, \\
d^{(1,2)}(N, 1)= & \frac{35}{24}+\frac{5}{2 \pi^{2}}-\frac{7 \zeta(3)}{4 \pi^{2}} \\
& +\left(\frac{15}{16}-\frac{1}{2 \pi^{2}}+\frac{7 \zeta(3)}{4 \pi^{2}}\right) N+\frac{7}{32} N^{2} .
\end{aligned}
$$


We note that the large-order asymptotics have the parameter $n=4$ in the conventions delineated in Eq. (1.9) because of the additional two fields that have to be inserted in view of the mass derivative.

\section{B. Interpretation of the results}

In this article, we have laid the groundwork for the accurate systematic analysis of the large-order behavior of perturbation theory for the correlation functions in the $\mathrm{N}$-vector model. Our paradigm is that once the number of loops in a Feynman diagram becomes very large, the largeorder behavior of the $N$-vector model is determined by classical field configurations (instantons), which determine the cut of the correlation functions for negative coupling. They act as a second saddle point of the Euclidean action. The (longitudinal) fluctuation operator around the saddle point has one negative eigenvalue, commensurate with the imaginary square root of the determinant of this operator.

Through the evaluation of corrections to the classical configurations, we are able to evaluate corrections of relative order $g$ to the correlation functions, which, via dispersion relations, immediately lead to the corrections of relative order $1 / K$ to the perturbative coefficients. The connection is elucidated in great detail in Sec. IB.

In all cases, the leading term of the order- $g$ correction, in the large- $N$ limit, is given by the multiplicative correction to the partition function term, i.e., due to the factor $\mathcal{F}_{\mathcal{Z}}$ given in Eq. (3.19). One might wonder why the term of order $N^{2}$ in the correction to the partition function constitutes the universal leading correction in the large- $N$ limit. To understand this phenomenon, let us consider the computational origin of the corrections. The leading term in a correlation function (for the imaginary part) is given by a term in which one replaces all field configurations by instantons. Then, a set of universal corrections is obtained when one keeps the instanton field configurations inside the main integrand but considers the corrections due to the field Jacobian, and due to the effective action around the instanton, which, together, give rise to the universal correction factor $\mathcal{F}_{\mathcal{Z}}$ given in Eq. (3.19).

Let us now consider the additional corrections obtained when one replaces, instead, one of the instanton field configurations by a fluctuation. Then, for the order- $g$ corrections to the imaginary part of the partition function, one has to combine the fluctuation with the factor $\mathcal{F}_{\mathcal{J}}$ from Eq. (3.25). This combination leads, at most, with regard to $N$, to the product of a longitudinal fluctuation factor, which carries no $N$, and one additional factor $N$ due to the transverse fluctuations encoded in $\underline{\chi}^{2}=\chi_{\mathrm{L}}^{2}+\underline{\chi}_{\mathrm{T}}^{2}$. Finally, replacing two instanton configurations by fluctuations, one obtains at most a single factor of $N$, generated by a term proportional to $\chi^{2}$. The universality of the large- $N$ limit of the correction terms can be justified based on the decoupling of expectation values of fields at different spacetime points in the limit of large $N$, as explained in the text following Eq. (2.2) of Ref. [26] and Chap. 14 of Ref. [27]. In fact, according to formulas given in Chap. 14 of Ref. [27], critical exponents reach universal values in the large- $N$ limit and depend only on the spatial dimension of the system.

Our expressions for the two-point correlation function, for its second derivative, for the four-point correlation function and for the two-point correlation function with a wigglet insertion, computed at zero momentum, were obtained by integrating the corresponding correlation function in coordinate space over the difference of all its coordinate with respect to one of them. Incidentally, it is interesting to note that this procedure is completely equivalent to an alternative procedure where one fixes one of the time coordinates in a correlation function to zero, and integrates over all the others. This equivalence holds due to the time translation invariance of the correlation functions. One of the most important additional conclusions of the current article is that, for the correlation functions, the two-loop corrections to the imaginary part, of relative order $g$, have a much more complex analytic structure as compared to those of the ground-state energy.

A remark on our notation is in order. We apologize to the reader for the many $G$ 's in our paper. Generic $n$-point Green functions are denoted by the sans-serif $\mathrm{G}$, according to Eq. (3.13). We also recall that $\mathcal{G}_{\alpha \beta}$, according to Eq. (3.15), is the imaginary part of the two-point function, while $\mathcal{G}_{\alpha \beta \gamma \delta}$, according to Eq. (3.63), is the imaginary part of the fourpoint function. Incidentally, we also have the terms $\mathcal{R}_{i}$ $(i=1, \ldots, 7)$ for the contributions to the two-point function at zero momentum transfer, according to Eq. (3.48). Seven corrections are also incurred for the contributions to the derivative of the two-point function, summarized in the terms $\mathcal{S}_{i}$ [see Eq. (3.54)]. For the four-point function one defines the scalar Green function $\mathbf{G}$ according to Eq. (3.76). Finally, we have the seven terms $\mathcal{T}_{i}(i=1, \ldots, 7)$ for the four-point function at zero momentum transfer, according to Eq. (3.79), and the seven terms $\mathcal{U}_{i}(i=1, \ldots, 7)$ for the two-point function with a wigglet insertion, according to Eq. (3.123).

A remark on the character of the factorial divergence of the perturbation series is in order. According to Eq. (1.9), all factorially divergent series calculated in this work are alternating in large order, in view of the factor $(-1 / A)^{K}$ in Eq. (1.9), where $A=4 / 3$. Indeed, it is known that perturbation series in $\phi^{4}$ theories are factorially divergent, Borel summable series [6-8,28-30]. In a more general context, such series constitute the conceptually simplest manifestation of so-called resurgent expansions (transseries), which have recently been shown to lead to adequate representations of physical quantities of interest in a number of mathematical investigations and in field theories [13-15,31-35]. 
In principle, based on the results presented in the current paper, one could go further and calculate the large-order behavior of the renormalization constants of the onedimensional $O(N)$ field theory (see Sec. B). We recall that the renormalization constants $Z_{\phi}$ (wave function), $Z_{\phi^{2}}$ (wigglet insertion), $Z_{g}$ (coupling constant), and $\delta m^{2}$ (mass counterterm) are determined by the renormalization conditions imposed on the vertex functions, which can be obtained from the correlation functions (calculated here) via a Legendre transformation. The renormalization constants, in turn, determine the large-order behavior of the beta function $\beta(g)$, the anomalous dimension function $\eta(g)$, and the correlation length function $\eta_{2}(g)$, which enter the Callan-Symanzik equation. The Callan-Symanzik equation is a $\mathrm{RG}$ equation fulfilled by the vertex functions of the theory (a minireview on this aspect is given in Appendix B). However, in one dimension, we refrain from engaging in this endeavor because of the absence of a second-order phase transition due to the low dimensionality of the system under study, which prevents the system from undergoing a phase transition to the low-temperature phase. In higher dimensions, the critical exponents can be studied on the basis of the Callan-Symanzik equation [9-12].

One of the main conclusions of the current paper is that the calculation of corrections to the large-order growth of perturbation theory for correlation functions, beyond the plain calculation of the partition function, is possible for field theories with a nontrivial internal structure [here, the $O(N)$ symmetry group]. The results presented here are a first step toward the evaluation of subleading corrections to the factorial asymptotics of perturbative coefficients in perturbative field theory, for physical quantities of interest beyond the partition function. The evaluation of subleading corrections to the large-order asymptotics of Feynman diagram coefficients in large loop-order provides us with an alternative method to enhance our understanding of the predictive limits of field theory. The ultimate goal of the endeavor is to "interpolate" between (necessarily finiteorder) perturbative Feynman diagram calculations and the large-order asymptotics (about "infinite loop" order), the latter being enhanced by the evaluation of the subleading corrections about the instantons. The latter, in turn, lead to corrections of relative order $1 / K$ (two-loop order discussed here) to the large-order behavior of perturbation theory. The generalization to relative order $1 / K^{2}$ (four-loop order about the instanton) and the consideration of field theories in higher dimensions are the natural next steps in this program and are currently under investigation.

\section{ACKNOWLEDGMENTS}

This work has been supported by the National Science Foundation (Grant No. PHY-1710856) and by the Swedish Research Council (Grant No. 638-2013-9243). Support from the Simons Foundation (Grant No. 454949) is also gratefully acknowledged.

\section{APPENDIX A: INTEGRAL TABLE}

We first list integrals that involve only the instanton,

$$
\begin{aligned}
& H_{1}=\int \mathrm{d} t \xi_{\mathrm{cl}}(t)=\sqrt{2} \pi, \\
& H_{2}=\int \mathrm{d} t\left[\xi_{\mathrm{cl}}(t)\right]^{2}=4, \\
& H_{3}=\int \mathrm{d} t t^{2} \xi_{\mathrm{cl}}(t)=\frac{\pi^{3}}{2 \sqrt{2}} .
\end{aligned}
$$

Integrals involving the subtracted longitudinal and transverse propagators read as follows:

$$
\begin{aligned}
I_{1} & =\int \mathrm{d} t_{1} \int \mathrm{d} t_{2}\left[\boldsymbol{\Delta}_{\mathrm{L}}\left(t_{1}, t_{2}\right)-\boldsymbol{\Delta}_{0}\left(t_{1}, t_{2}\right)\right]=-4, \\
I_{2} & =\int \mathrm{d} t_{1} \int \mathrm{d} t_{2}\left[\boldsymbol{\Delta}_{\mathrm{T}}\left(t_{1}, t_{2}\right)-\boldsymbol{\Delta}_{0}\left(t_{1}, t_{2}\right)\right] \\
& =1-\frac{\pi^{2}}{4}-\frac{7}{2} \zeta(3) .
\end{aligned}
$$

In addition, we have integrals involving longitudinal and transverse propagators and instantons,

$$
\begin{aligned}
& J_{1}=\int \mathrm{d} t_{1} \xi_{\mathrm{cl}}\left(t_{1}\right) \int \mathrm{d} t_{2} \Delta_{\mathrm{L}}\left(t_{1}, t_{2}\right)=0, \\
& J_{2}=\int \mathrm{d} t_{1} \ddot{\xi}_{\mathrm{cl}}\left(t_{1}\right) \int \mathrm{d} t_{2} \boldsymbol{\Delta}_{\mathrm{L}}\left(t_{1}, t_{2}\right)=\frac{\pi}{\sqrt{2}}, \\
& J_{3}=\int \mathrm{d} t_{1} \xi_{\mathrm{cl}}\left(t_{1}\right) \Delta_{\mathrm{L}}\left(t_{1}, t_{1}\right) \int \mathrm{d} t_{2} \Delta_{\mathrm{L}}\left(t_{1}, t_{2}\right)=\frac{\pi}{4 \sqrt{2}}, \\
& J_{4}=\int \mathrm{d} t_{1} \xi_{\mathrm{cl}}\left(t_{1}\right) \boldsymbol{\Delta}_{\mathrm{T}}\left(t_{1}, t_{1}\right) \int \mathrm{d} t_{2} \boldsymbol{\Delta}_{\mathrm{L}}\left(t_{1}, t_{2}\right)=\frac{\pi}{8 \sqrt{2}} .
\end{aligned}
$$

Integrals involving the subtracted longitudinal and transverse propagators, and powers of the Euclidean time variable, are needed for the second derivative of the two-point function,

$$
\begin{aligned}
K_{1} & =\int \mathrm{d} t_{1} \int \mathrm{d} t_{2} t_{1}^{2}\left[\boldsymbol{\Delta}_{\mathrm{L}}\left(t_{1}, t_{2}\right)-\boldsymbol{\Delta}_{0}\left(t_{1}, t_{2}\right)\right] \\
& =-\frac{\pi^{2}}{3}, \\
K_{2} & =\int \mathrm{d} t_{1} \int \mathrm{d} t_{2} t_{1}^{2}\left[\boldsymbol{\Delta}_{\mathrm{T}}\left(t_{1}, t_{2}\right)-\boldsymbol{\Delta}_{0}\left(t_{1}, t_{2}\right)\right] \\
& =2+\frac{\pi^{2}}{12}-\frac{\pi^{4}}{16}-\frac{7}{24} \pi^{2} \zeta(3)-\frac{31}{2} \zeta(5) .
\end{aligned}
$$

Furthermore, we have the following integrals with mixed contributions: 


$$
\begin{aligned}
\tilde{K}_{1} & =\int \mathrm{d} t_{1} \int \mathrm{d} t_{2} t_{1} t_{2}\left[\boldsymbol{\Delta}_{\mathrm{L}}\left(t_{1}, t_{2}\right)-\boldsymbol{\Delta}_{0}\left(t_{1}, t_{2}\right)\right] \\
& =-\frac{7 \pi^{2}}{12}-1-\frac{21}{2} \zeta(3), \\
\tilde{K}_{2} & =\int \mathrm{d} t_{1} \int \mathrm{d} t_{2} t_{1} t_{2}\left[\boldsymbol{\Delta}_{\mathrm{T}}\left(t_{1}, t_{2}\right)-\boldsymbol{\Delta}_{0}\left(t_{1}, t_{2}\right)\right] \\
& =-1+\frac{\pi^{2}}{12}-\frac{7}{24} \pi^{2} \zeta(3)+\frac{31}{4} \zeta(5) .
\end{aligned}
$$

Among all integrals considered, the integrals $K_{2}$ and $\tilde{K}_{2}$ are by far the most difficult to evaluate.

One may derive a relatively compact representation for $K_{2}$ via the substitutions $t_{2}=2 \ln (v)$, followed by the $t_{2}$ integral, then followed by $t_{1}=2 \ln (u)$, and $u=\sqrt{x}$, so one would effectively substitute $t_{1}=\ln (x)$. This results in

$$
\begin{aligned}
K_{2}= & \int_{0}^{\infty} \mathrm{d} x\left\{-\frac{\ln ^{2}(x)}{x\left(x^{2}+1\right)}-\frac{\ln ^{2}(x)\left(x^{2}-1\right) \arctan (x)}{x^{2}\left(x^{2}+1\right)}\right. \\
& \left.-\frac{\mathrm{i} \ln ^{2}(x)}{1+x^{2}}\left[\operatorname{Li}_{2}(-\mathrm{i} x)-\mathrm{Li}_{2}(\mathrm{i} x)\right]\right\} .
\end{aligned}
$$

This representation is seen to involve Legendre's $\chi_{2}$ function [36,37],

$$
\chi_{2}(z)=\frac{1}{2}\left(\mathrm{Li}_{2}(z)-\mathrm{Li}_{2}(-z)\right)
$$

Formula (A11) was found by the PSLQ algorithm [20-23]. One can form the combinations

$$
\begin{aligned}
\bar{K}_{1} & =\int \mathrm{d} t_{1} \int \mathrm{d} t_{2}\left(t_{1}-t_{2}\right)^{2}\left[\boldsymbol{\Delta}_{\mathrm{L}}\left(t_{1}, t_{2}\right)-\boldsymbol{\Delta}_{0}\left(t_{1}, t_{2}\right)\right] \\
& =2\left(K_{1}-\tilde{K}_{1}\right)=2+\frac{\pi^{2}}{2}+21 \zeta(3), \\
\bar{K}_{2} & =\int \mathrm{d} t_{1} \int \mathrm{d} t_{2}\left(t_{1}-t_{2}\right)^{2}\left[\boldsymbol{\Delta}_{\mathrm{T}}\left(t_{1}, t_{2}\right)-\boldsymbol{\Delta}_{0}\left(t_{1}, t_{2}\right)\right] \\
& =2\left(K_{2}-\tilde{K}_{2}\right)=6-\frac{\pi^{4}}{8}-\frac{93}{2} \zeta(5) .
\end{aligned}
$$

We have additional reference integrals with a second derivative insertion, which are important for the calculation of the derivative $\partial^{2} G / \partial p^{2}$, at $p=0$,

$$
\begin{aligned}
& L_{1}=\int \mathrm{d} t_{1} \xi_{\mathrm{cl}}\left(t_{1}\right) \int \mathrm{d} t_{2} t_{2}^{2} \Delta_{\mathrm{L}}\left(t_{1}, t_{2}\right)=\frac{\pi^{3}}{2 \sqrt{2}}, \\
& L_{2}=\int \mathrm{d} t_{1} \ddot{\xi}_{\mathrm{cl}}\left(t_{1}\right) \int \mathrm{d} t_{2} t_{2}^{2} \Delta_{\mathrm{L}}\left(t_{1}, t_{2}\right)=\frac{3 \pi^{3}}{4 \sqrt{2}},
\end{aligned}
$$

$$
\begin{aligned}
L_{3} & =\int \mathrm{d} t_{1} \xi_{\mathrm{cl}}\left(t_{1}\right) \boldsymbol{\Delta}_{\mathrm{L}}\left(t_{1}, t_{1}\right) \int \mathrm{d} t_{2} t_{2}^{2} \boldsymbol{\Delta}_{\mathrm{L}}\left(t_{1}, t_{2}\right) \\
& =\frac{5 \pi^{3}}{16 \sqrt{2}}-\frac{\pi}{12 \sqrt{2}}, \\
L_{4} & =\int \mathrm{d} t_{1} \xi_{\mathrm{cl}}\left(t_{1}\right) \boldsymbol{\Delta}_{\mathrm{T}}\left(t_{1}, t_{1}\right) \int \mathrm{d} t_{2} t_{2}^{2} \boldsymbol{\Delta}_{\mathrm{L}}\left(t_{1}, t_{2}\right) \\
& =\frac{9 \pi^{3}}{32 \sqrt{2}} .
\end{aligned}
$$

For the wigglet insertion, i.e., the Green function $\mathcal{G}^{(1,2)}$ at zero momentum, we also need the integrals

$$
\begin{aligned}
M_{1} & =\int \mathrm{d} t_{1} \xi_{\mathrm{cl}}\left(t_{1}\right) \int \mathrm{d} t_{2} \xi_{\mathrm{cl}}\left(t_{2}\right) \Delta_{\mathrm{L}}\left(t_{1}, t_{2}\right)=-1, \\
M_{2} & =\int \mathrm{d} t_{1} \ddot{\xi}_{\mathrm{cl}}\left(t_{1}\right) \int \mathrm{d} t_{2} \xi_{\mathrm{cl}}\left(t_{2}\right) \Delta_{\mathrm{L}}\left(t_{1}, t_{2}\right)=1, \\
M_{3} & =\int \mathrm{d} t_{1} \xi_{\mathrm{cl}}\left(t_{1}\right) \Delta_{\mathrm{L}}\left(t_{1}, t_{1}\right) \int \mathrm{d} t_{2} \xi_{\mathrm{cl}}\left(t_{2}\right) \Delta_{\mathrm{L}}\left(t_{1}, t_{2}\right) \\
& =\frac{1}{36} \\
M_{4} & =\int \mathrm{d} t_{1} \xi_{\mathrm{cl}}\left(t_{1}\right) \Delta_{\mathrm{T}}\left(t_{1}, t_{1}\right) \int \mathrm{d} t_{2} \xi_{\mathrm{cl}}\left(t_{2}\right) \Delta_{\mathrm{L}}\left(t_{1}, t_{2}\right) \\
& =-\frac{1}{4}
\end{aligned}
$$

In view of the confluence of arguments of the propagators in the wigglet insertion, additional integrals are required,

$$
\begin{aligned}
& N_{1}=\int \mathrm{d} t_{1}\left[\boldsymbol{\Delta}_{\mathrm{L}}\left(t_{1}, t_{1}\right)-\boldsymbol{\Delta}_{0}\left(t_{1}, t_{1}\right)\right]=-\frac{7}{6}, \\
& N_{2}=\int \mathrm{d} t_{1}\left[\boldsymbol{\Delta}_{\mathrm{T}}\left(t_{1}, t_{1}\right)-\boldsymbol{\Delta}_{0}\left(t_{1}, t_{1}\right)\right]=-\frac{1}{2} .
\end{aligned}
$$

Finally, we also need the integral

$$
J_{1}^{(0)}=\int \mathrm{d} t_{1} \xi_{\mathrm{cl}}\left(t_{1}\right) \int \mathrm{d} t_{2} \boldsymbol{\Delta}_{0}\left(t_{1}, t_{2}\right)=\sqrt{2} \pi,
$$

which is obtained from (A6) by the replacement of the longitudinal propagator with its free counterpart.

\section{APPENDIX B: CALLAN-SYMANZIK EQUATION}

In principle, the application of the Callan-Symanzik equation $[24,25]$ to the calculation of critical exponents has been described in a number of monographs [27,30,38,39]. However, to put the calculations reported here into perspective, we should include a few remarks regarding the relation of the quantities calculated here to the RG functions that enter the Callan-Symanzik equation. 
The Callan-Symanzik equation is a RG equation fulfilled by one-particle irreducible vertex functions $\Gamma^{(a, n)}$, derived via Legendre transformation from the connected correlation functions (see Chap. 7 of Ref. [30]).

The Callan-Symanzik equation is obtained by differentiating the vertex functions with respect to the renormalized mass parameter while holding the bare parameters constant and reads as follows:

$$
\begin{aligned}
& \left.\left[m_{R} \frac{\partial}{\partial m_{R}}+\beta\left(g_{R}\right) \frac{\partial}{\partial g_{R}}-\frac{n}{2} \eta\left(g_{R}\right)-a \eta_{2}\left(g_{R}\right)\right)\right] \\
& \Gamma_{R}^{(a, n)}\left(\left\{\vec{q}_{i}\right\}_{i=1}^{a} ;\left\{\vec{p}_{i}\right\}_{i=1}^{n} ; m_{R}, g_{R}\right) \\
& \quad=\left[2-\eta\left(g_{R}\right)\right] \Gamma_{R}^{(a+1, n)}\left(\left\{\vec{q}_{i}\right\}_{i=1}^{a}, \overrightarrow{0} ;\left\{\vec{p}_{i}\right\}_{i=1}^{n} ; m_{R}, g_{R}\right) .
\end{aligned}
$$

Here, $\Gamma_{R}^{(a, n)}$ is the $n$-point vertex function with $a$ wigglet insertions. In the term with $\Gamma_{R}^{(a+1, n)}$, we have to set the momentum argument corresponding to the wigglet insertion number $a+1$ (the one created by the action of the operator $\partial / \partial m_{R}^{2}$ on $\Gamma_{R}^{(a, n)}$ ) to zero. As already mentioned, the vertex functions are obtained from the connected correlation functions via a Legendre transformation. For example, the vertex function $\Gamma^{(2)}=\Gamma^{(0,2)}$ is the inverse of the two-point correlation function $W^{(2)}$ [see the discussion following Eq. (7.80) of Ref. [30] ]. The four-point function $\Gamma^{(4)}=\Gamma^{(0,4)}$ is obtained from $W^{(4)}$ via "amputation" of the external legs and sign inversion [see the discussion following Eq. (7.80) of Ref. [30], or Eq. (4.24) of Ref. [39] ].

Bare (index zero) and renormalized vertex functions are related by [see Eq. (10.20) of Ref. [30] ]

$$
\begin{aligned}
\Gamma_{0}^{(a, n)} & \left(\left\{\vec{q}_{i}\right\}_{i=1}^{a} ;\left\{\vec{p}_{i}\right\}_{i=1}^{n} ; m_{0}, g_{0}\right) \\
& =Z_{\phi}^{-n / 2}\left(\frac{Z_{\phi}}{Z_{\phi^{2}}}\right)^{a} \Gamma_{R}^{(a, n)}\left(\left\{\vec{q}_{i}\right\}_{i=1}^{a} ;\left\{\vec{p}_{i}\right\}_{i=1}^{n} ; m_{R}, g_{R}\right) .
\end{aligned}
$$

Here, $Z_{\phi}$ is the wave function renormalization, $Z_{\phi^{2}}$ is the renormalization of the wigglet insertion, and the bare and renormalized mass parameters are related by

$$
m_{0}^{2}=\left(m_{R}^{2}+\delta m^{2}\right) / Z_{\phi},
$$

where $\delta m^{2}$ is the mass counterterm.

The wave function renormalization constant $Z_{\phi^{2}}$ is fixed by the condition

$$
\left.\frac{\partial}{\partial p_{1}^{2}} \Gamma_{R}^{(2)}\left(\vec{p}_{1}, \vec{p}_{2} ; m_{R}^{2}, g_{R}\right)\right|_{\vec{p}_{i}=\overrightarrow{0}}=1
$$

The renormalization condition

$$
\left.\Gamma_{R}^{(2)}\left(\vec{p}_{1}, \vec{p}_{2} ; m_{R}^{2}, g_{R}\right)\right|_{\vec{p}_{i}=\overrightarrow{0}}=m_{R}^{2}
$$

fixes the mass counterterm $\delta m^{2}$. The coupling constant renormalization constant $Z_{g}$ is fixed by the condition

$$
\left.\Gamma_{R}^{(4)}\left(\vec{p}_{1}, \vec{p}_{2} ; \vec{p}_{3}, \vec{p}_{4}, m_{R}^{2}, g_{R}\right)\right|_{\vec{p}_{i}=\overrightarrow{0}}=m_{R}^{4-D} g_{R},
$$

where $g_{R}$ is the dimensionless, renormalized coupling, and $D$ is the spatial dimension. Finally, the wigglet insertion renormalization constant $Z_{\phi^{2}}$ is determined by

$$
\left.\Gamma_{R}^{(1,2)}\left(\vec{q} ; \vec{p}_{1}, \vec{p}_{2} ; m_{R}^{2}, g_{R}\right)\right|_{\vec{p}_{i}, \vec{q}=0}=1 .
$$

The renormalization-group functions are obtained as follows:

$$
\begin{aligned}
\beta\left(g_{R}\right) & =-(4-D)\left[\frac{\partial}{\partial g_{R}} \ln \left(g_{R} \frac{Z_{g}\left(g_{R}\right)}{Z_{\phi}\left(g_{R}\right)^{2}}\right)\right]^{-1}, \\
\eta(g) & =\beta\left(g_{R}\right) \frac{\partial}{\partial g_{R}} \ln \left(Z_{\phi}\right), \\
\eta_{2}(g) & =\beta\left(g_{R}\right) \frac{\partial}{\partial g_{R}} \ln \left(\frac{Z_{\phi^{2}}}{Z_{\phi}}\right) .
\end{aligned}
$$

From these relations, one calculates the critical value $g^{*}$ determined by the condition $\beta\left(g^{*}\right)=0$ which determines the infrared non-Gaussian fixed point of the RG flow, which is approached by the system because of the relevance of the $\phi^{4}$ interaction in dimensions lower than four. Critical exponents are determined by the values of the RG functions at the critical point, $\eta=\eta\left(g^{*}\right)$ and $\eta_{2}=\eta_{2}\left(g^{*}\right)$, via wellknown hyperscaling relations. For example, the critical exponent $\nu$ of the correlation length is determined as $\nu=1 /\left(2+\eta_{2}\right)$, while the critical exponent $\alpha$ of the heat capacity is $\alpha=2-D \nu$, and the critical exponent $\gamma$ of the magnetic susceptibility is $\gamma=\nu(2-\eta)$.
[1] F. J. Dyson, Divergence of perturbation theory in quantum electrodynamics, Phys. Rev. 85, 631 (1952).

[2] C. M. Bender and T. T. Wu, Anharmonic oscillator, Phys. Rev. 184, 1231 (1969).
[3] C. M. Bender and T. T. Wu, Large-Order Behavior of Perturbation Theory, Phys. Rev. Lett. 27, 461 (1971).

[4] C. M. Bender and T. T. Wu, Anharmonic oscillator. II. A study of perturbation theory in large order, Phys. Rev. D 7, 1620 (1973). 
[5] E. Brézin, G. Parisi, and J. Zinn-Justin, Perturbation theory at large orders for a potential with degenerate minima, Phys. Rev. D 16, 408 (1977).

[6] E. Brézin, J. C. Le Guillou, and J. Zinn-Justin, Perturbation theory at large order. I. The $\phi^{2 N}$ interaction, Phys. Rev. D 15, 1544 (1977).

[7] E. Brézin, J. C. Le Guillou, and J. Zinn-Justin, Perturbation theory at large order. II. Role of the vacuum instability, Phys. Rev. D 15, 1558 (1977).

[8] E. Brézin and G. Parisi, Critical exponents and large-order behavior of perturbation theory, J. Stat. Phys. 19, 269 (1978); Reprinted in E. Brézin and G. Parisi, Field Theory, Disorder and Simulations, edited by G. Parisi (World Scientific, Singapore, 1992), pp. 119-142.

[9] J. C. LeGuillou and J. Zinn-Justin, Critical Exponents for the $n$-Vector Model in Three Dimensions from Field Theory, Phys. Rev. Lett. 39, 95 (1977).

[10] J. C. Le Guillou and J. Zinn-Justin, Critical exponents from field theory, Phys. Rev. B 21, 3976 (1980).

[11] Large-Order Behaviour of Perturbation Theory, edited by J. C. Le Guillou and J. Zinn-Justin (North-Holland, Amsterdam, 1990).

[12] R. Guida and J. Zinn-Justin, Critical Exponents of the $N$ vector model, J. Phys. A 31, 8103 (1998).

[13] U. D. Jentschura and J. Zinn-Justin, Instanton effects in quantum mechanics and resurgent expansions, Phys. Lett. B 596, 138 (2004).

[14] J. Zinn-Justin and U. D. Jentschura, Multi-instantons and exact results I: Conjectures, WKB expansions, and instanton interactions, Ann. Phys. (Amsterdam) 313, 197 (2004).

[15] J.Zinn-Justin and U. D. Jentschura, Multi-instantons and exact results II: Specific cases, higher-order effects, and numerical calculations, Ann. Phys. (Amsterdam) 313, 269 (2004).

[16] U. D. Jentschura, A. Surzhykov, and J. Zinn-Justin, Unified Treatment of Even and Odd Anharmonic Oscillators of Arbitrary Degree, Phys. Rev. Lett. 102, 011601 (2009).

[17] U. D. Jentschura, A. Surzhykov, and J. Zinn-Justin, Multiinstantons and exact results III: Unified description of the resonances of even and odd anharmonic oscillators, Ann. Phys. (Amsterdam) 325, 1135 (2010).

[18] U. D. Jentschura and J. Zinn-Justin, Multi-instantons and exact results IV: Path integral formalism, Ann. Phys. (Amsterdam) 326, 2186 (2011).

[19] E. M. Malatesta, G. Parisi, and T. Rizzo, Two-loop corrections to large order behavior of $\varphi^{4}$ theory, Nucl. Phys. B922, 293 (2017).

[20] H. R. P. Ferguson and D. H. Bailey, A polynomial time, numerically stable integer relation algorithm, RNR Technical Report No. RNR-91-032, 1992.
[21] D. H. Bailey and S. Plouffe, Recognizing numerical constants, in Proceedings of the Workshop on Organic Mathematics, Burnaby, BC, edited by J. Borwein, P. Borwein, L. Joergenson, and R. Corless (American Mathematical Society, Philadelphia, PA, 1997), pp. 73-88.

[22] H. R. P. Ferguson, D. H. Bailey, and S. Arno, Analysis of PSLQ, an integer relation finding algorithm, Math. Comput. 68, 351 (1999).

[23] D. H. Bailey and D. Broadhurst, Integer relation detection, Math. Comput. 70, 1719 (2001).

[24] J. Callan, Broken scale invariance in scalar field theory, Phys. Rev. D 2, 1541 (1970).

[25] K. Symanzik, Small distance behaviour in field theory and power counting, Commun. Math. Phys. 18, 227 (1970).

[26] M. Moshe and J. Zinn-Justin, Quantum field theory in the large $N$ limit: A review, Phys. Rep. 385, 69 (2003).

[27] J. Zinn-Justin, Phase Transitions and Renormalisation Group (Oxford University Press, New York, 2007).

[28] G. Parisi, Singularities of the Borel transform in renormalizable theories, Phys. Lett. 76B, 65 (1978).

[29] E. Caliceti, V. Grecchi, and M. Maioli, The distributional Borel summability and the large coupling $\phi^{4}$ lattice fields, Commun. Math. Phys. 104, 163 (1986).

[30] J. Zinn-Justin, Quantum Field Theory and Critical Phenomena (Oxford University Press, Oxford, 2002), 4th ed.

[31] F. Pham, Fonctions résurgentes implicites, C. R. Acad. Sci. Paris: Ser. I Math. 309, 999 (1989), https://mathscinet.ams .org/mathscinet-getitem?mr=1054521.

[32] E. Delabaere and F. Pham, Eigenvalues of complex Hamiltonians with PT-symmetry. I, Phys. Lett. A 250, 25 (1998).

[33] E. Delabaere and F. Pham, Eigenvalues of complex Hamiltonians with PT-symmetry. II, Phys. Lett. A 250, 29 (1998).

[34] B. Candelpergher, J. C. Nosmas, and F. Pham, Approche de la Résurgence (Hermann, Paris, 1993).

[35] An overview of recent activity in the field was given at the conference on "Resurgence and Transseries in Quantum, Gauge and String Theories" held at CERN from 30-JUN-2014 to 04-JUL-2014.

[36] L. Lewin, Polylogarithms and Associated Functions (North-Holland, New York, 1981).

[37] A. M. Legendre, Exercises de Calcul Intégral (Courcier, Paris, 1811).

[38] G. Parisi, Statistical Field Theory (Perseus, Reading, Massachusetts, 1988).

[39] H. Kleinert and V. Schulte-Frohlinde, Critical Properties of $\phi^{4}$-Theories (World Scientific, Singapore, 2007). 